\title{
Bose-Einstein condensation in quantum magnets
}

\author{
Vivien Zapf ${ }^{*}$ and Marcelo Jaime ${ }^{\dagger}$ \\ National High Magnetic Field Laboratory, Materials Physics and Applications Division, \\ Los Alamos National Laboratory, Los Alamos, New Mexico 87545, USA
}

\author{
C. D. Batista
}

Theoretical Division, T-4 and CNLS, Los Alamos National Laboratory, Los Alamos, New Mexico 87545, USA

(published 15 May 2014)

\begin{abstract}
This article reviews experimental and theoretical work on Bose-Einstein condensation in quantum magnets. These magnets are natural realizations of gases of interacting bosons whose relevant parameters such as dimensionality, lattice geometry, amount of disorder, nature of the interactions, and particle concentration can vary widely between different compounds. The particle concentration can be easily tuned by applying an external magnetic field which plays the role of a chemical potential. This rich spectrum of realizations offers a unique possibility for studying the different physical behaviors that emerge in interacting Bose gases from the interplay between their relevant parameters. The plethora of other bosonic phases that can emerge in quantum magnets, of which the Bose-Einstein condensate is the most basic ground state, is reviewed. The compounds discussed in this review have been intensively studied in the last two decades and have led to important contributions in the area of quantum magnetism. In spite of their apparent simplicity, these systems often exhibit surprising behaviors. The possibility of using controlled theoretical approaches has triggered the discovery of unusual effects induced by frustration, dimensionality, or disorder.
\end{abstract}

DOI: $10.1103 /$ RevModPhys.86.563

PACS numbers: 75.40.-s, 65.40.-b, 75.10.Jm, 74.40.Kb

\section{CONTENTS}

I. Introduction

II. Theory

A. Mappings between spin and itinerant-particle systems

B. Uniaxial symmetry breaking and superfluid currents

C. Quantum criticality

1. BEC quantum critical points

2. Dimensional reduction

D. Theoretical approaches

1. Perturbation theory

2. Generalized spin-wave theory

3. Dilute gas

4. Numerical approaches

E. Theoretical summary

III. Experiments

A. Compounds

B. Measurements

1. Magnetization

2. Specific heat

3. Magnetocaloric effect

4. Thermal conductivity

5. Evidence of quantum fluctuations: Asymmetry of the phase diagram

6. Magnetic effects on the crystal lattice

7. Critical exponents for the thermal properties near the BEC QCP
563

567

567

569

570

570

572

574

574

577

579

581

582

583

583

585

586

588

589

592

593

594

596

\footnotetext{
*vzapf@lanl.gov

†mjaime@lanl.gov

"cdb@lanl.gov
}

8. Pressure-induced quantum critical points $\quad 598$

9. Elastic neutron scattering $\quad 599$

10. Inelastic neutron scattering $\quad 599$

11. Electron spin resonance 603

12. Nuclear magnetic resonance 604

IV. Magnetic Frustration 605

V. Future Directions and Conclusions 607

A. Disorder 607

B. Exotic states induced by frustration 608

C. Low dimensionality 608

D. Other exotic states 609

E. Conclusions $\quad 609$

Acknowledgments $\quad 610$

References $\quad 610$

\section{INTRODUCTION}

The idea of a Bose-Einstein condensate (BEC) was proposed in 1924 by Bose (1924) and Einstein (1924). Following the work of Bose on the statistics of photons (Bose, 1924), Einstein showed in his original paper that a noninteracting gas of bosons condenses into a coherent BEC in which a macroscopic number of bosons occupies the lowest-energy singleparticle state below a critical temperature $T_{c}$. In 1938 London suggested the connection between the superfluidity of ${ }^{4} \mathrm{He}$ and Bose-Einstein condensation (London, 1938). Superfluid ${ }^{4} \mathrm{He}$ is indeed the prototype of a BEC and it has played a crucial role in the development of the main physical concepts associated with this phase (Pitaevskii and Stringari, 2003). However, the strong interaction between ${ }^{4} \mathrm{He}$ atoms dramatically reduces the condensate fraction even at zero temperature (Svensson and Sears, 1987). This fact led to a new search for 
dilute Bose gases. Bose alkali-metal gases were the first example of very dilute and consequently weakly interacting BECs (Pethick and Smith, 2002), and the unambiguous observation of a BEC in a dilute gas of bosonic atoms did not occur until 1995, when the groups of Cornell and Wiemann and Ketterle independently observed this state in laser-cooled collections of cold atoms, leading to the Nobel prize (Anderson et al., 1995; Davis et al., 1995).

The main concepts and properties of BECs are reviewed in different texts (Nozieres and Pines, 1990; Griffin, Snoke, and Stringari, 1995; Dalfovo et al., 1999; Inguscio, Stringari, and Wieman, 1999; Leggett, 2001). In this paper we introduce and describe the properties of quantum magnets that are magnetic realizations of Bose gases.

Thirty years after the pioneering works of Bose and Einstein, Matsubara and Matsuda introduced an exact mapping between hard-core bosons and $S=1 / 2$ spins (Matsubara and Matsuda, 1956). From this mapping it became clear that interacting $S=1 / 2$ spin systems can be treated as gases of bosons with hard-core repulsion. This exact mapping can be generalized in different ways for higher-spin systems (Batista and Ortiz, 2001, 2004) and suggests that the BEC state can be realized in quantum magnets. The precise mapping is described in detail in the next section. Identifying that the spins can be mapped onto bosons is the first step toward realizing a BEC. The next step is to show that the bosons can condense (Batyev and Braginskii, 1984; Affleck, 1990, 1991; Giamarchi and Tsvelik, 1999; Nikuni et al., 2000; Nohadani et al., 2004; Giamarchi, Rüegg, and Tchernyshyov, 2008). The key property that separates systems of bosons that condense from those that do not is number conservation. Number conservation is naturally present in the case of cold atoms. In the case of magnetic systems, number conservation implies uniaxial symmetry of the spin environment. Since this is never strictly true for real magnets, the notion of BEC is always an idealization, but it turns out to be a good approximation for most of the materials that will be discussed in this review. We will see in the next sections that a spin Hamiltonian that has uniaxial symmetry commutes with the boson number operator. The local order parameter of an $X Y$ ordered magnet is a two-dimensional vector $\left(m_{x}, m_{y}\right)$ that has a magnitude and a phase. Similarly, the BEC order has also a magnitude and a phase. Therefore, it is not surprising that both orderings can be connected by a spin-particle transformation. This mapping between order parameters already suggests that the $z$ component of the local magnetization $m_{z}$ maps into the boson density. Therefore, the boson density of magnetic systems can be tuned with a magnetic field, which plays the role of a chemical potential. We will also see in the next sections that the magnetic-field-induced quantum critical point (QCP) that separates the quantum paramagnetic and the $X Y$-ordered states belongs to the BEC universality class. A partial list of materials where this phenomenon has been studied is shown in Table I.

There are several compelling reasons for studying BECs in quantum magnets. The study of BECs in quantum magnets has yielded experimental tests of the expected scaling laws of thermodynamic quantities near a BEC QCP (Sebastian et al., 2005; Tokiwa et al., 2006; Zapf et al., 2006; Kitada et al., 2007; Yamada et al., 2008; Yin et al., 2008). In addition, the magnetic field provides a simple and reversible tuning parameter to adjust the boson number in quantum magnets. As a function of magnetic field we drive the system from a state with no bosons to a state with a finite number of bosons, thereby creating a quantum phase transition driven by amplitude fluctuations of the BEC order parameter with dynamical exponent $z=2$. Finally, the effect of zero-point or quantum fluctuations on the single-boson mass can be observed by measuring different thermodynamic properties (Samulon et al., 2009; Kohama et al., 2011). A quantum phase transition that belongs to a different universality class with $z=1$ can also be induced in some of these systems by applying pressure. The single-boson mass becomes particularly small for quantum paramagnets that are close to the pressureinduced QCP, as described in Sec. III.B.5. Another reason to study $\mathrm{BEC}$ in quantum magnets is that, compared to $\mathrm{BEC}$ systems in cold atoms, the number of bosons is very large and the underlying lattice is very homogeneous, enabling us to study predictions of BEC theory in the thermodynamic limit. The bosons in quantum magnets are much lighter than in cold atoms, which leads to critical temperatures that range up to a few kelvin. The critical temperatures could even occur at ambient temperature, although BECs at such high temperatures have not been experimentally studied because it would likely require thermodynamic measurements at magnetic fields of several hundred tesla, which are not yet practical.

Observing the BEC state is just the first step. The identification of quantum magnets with Bose gases opens a rich new field of bosonic physics, of which the BEC is just the first and the most basic ground state. Unlike other realizations of Bose gases, magnetic realizations offer a natural way of producing single-boson dispersion relations with more than one minimum. This degeneracy of the single-particle ground state arises from competing exchange interactions that are common in frustrated quantum magnets. A natural consequence is the possibility of observing multi- $\mathbf{Q}$ BECs $(\mathbf{Q}$ is the wave vector of the single-particle ground state), which can correspond to exotic magnetic orderings, such as spontaneous vortex crystals (Kamiya and Batista, 2013). Bosons can also crystallize as in the case of $\mathrm{SrCu}_{2}(\mathrm{BO})_{3}$ (Rice, 2002). In analogy with other bosonic systems, the bosons in quantum magnets can be weakly or strongly interacting, ordered or disordered. Magnetic frustration can create a Bose metal (a type of spin liquid in the magnetic language). When disorder is introduced, Bose glasses can occur and the BEC undergoes a type of conducting-to-insulating transition analogous to Anderson localization for fermions.

Another compelling reason for describing $X Y$ magnets in terms of bosons is that we can borrow the entire formalism that has been developed for gases of bosons and use it to greatly simplify the theoretical description of the quantum magnets. In particular, dilute gases of bosons can be treated under control by using perturbation theory and expanding in the ratio between the scattering length and the mean distance between particles (this ratio is known as the lattice gas parameter). This approach is particularly useful for describing the physics near the magnetic-field-induced quantum critical point of many quantum magnets like the ones listed in Table I. It is important to emphasize that there are very few controlled approaches for treating low-spin magnetic systems. The dilute-gas approach 


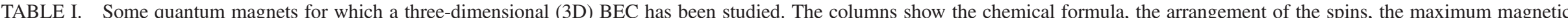

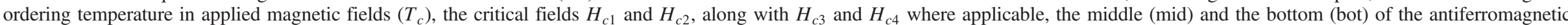

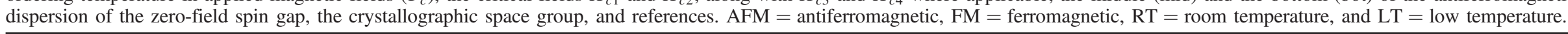

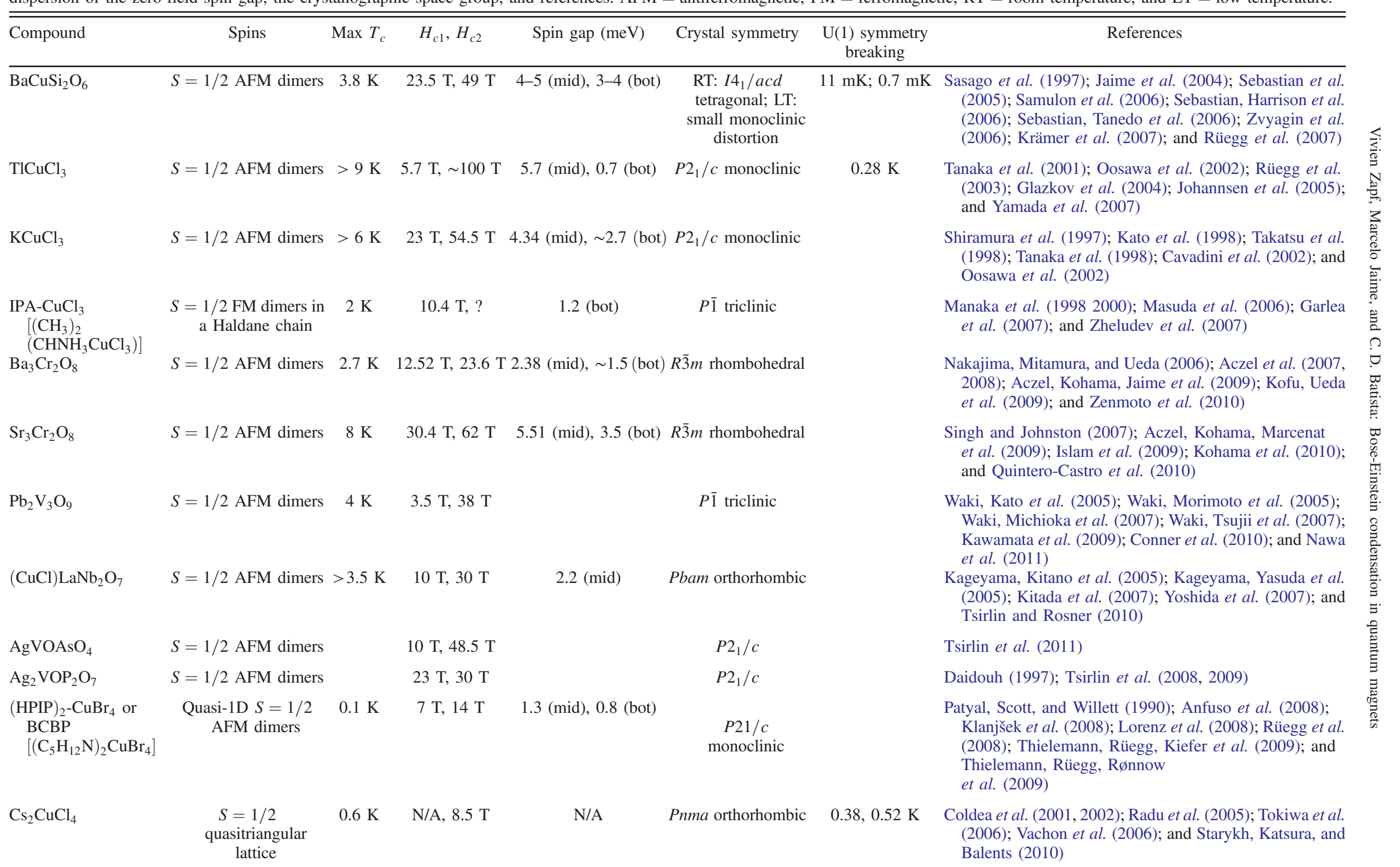

(Table continued) 


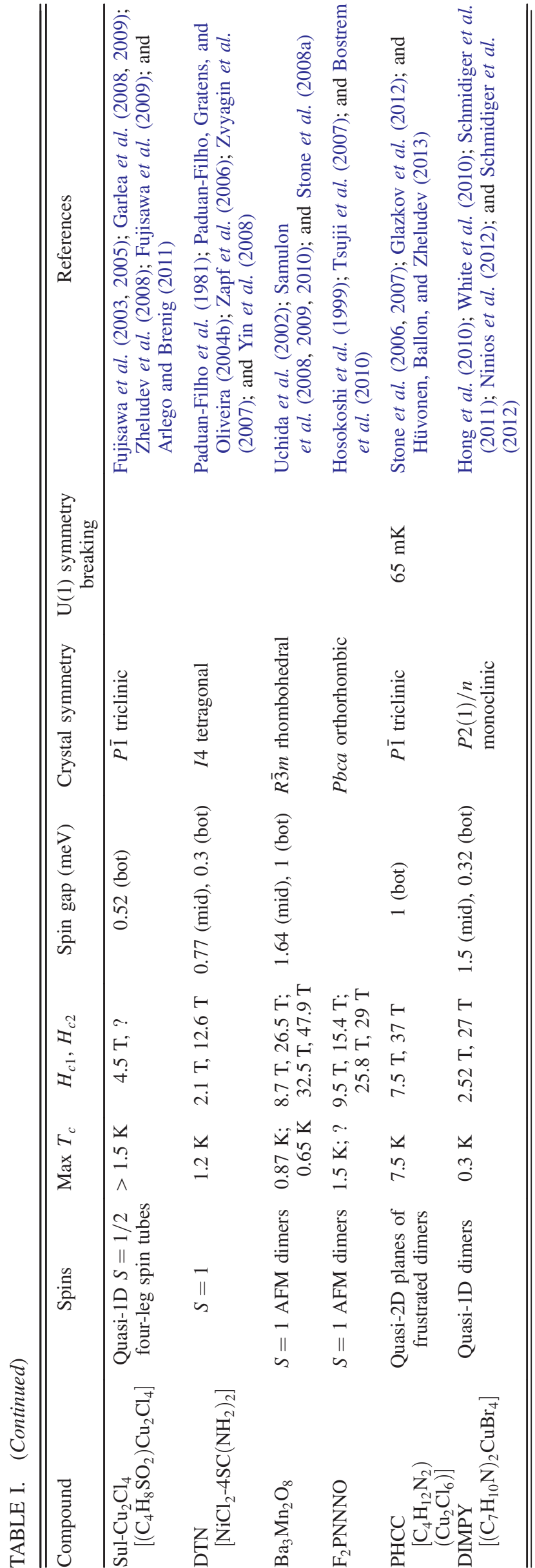

is then useful for calculating a number of key properties, including the nature of the field-induced magnetic ordering right above the critical field, the spectrum of excitations, and the dynamical and transport properties in the neighborhood of the QCP. Thus, there is a big advantage in describing these magnetic systems as a gas of bosons.

We should elaborate on the fact that boson number conservation, which underlies the BEC state, is an approximation in the case of quantum magnets, as it is in many other experimental realizations of BECs. In the magnetic language, the boson number corresponds to the longitudinal magnetization along the magnetic field, and the number conservation corresponds to uniaxial symmetry (continuous symmetry of global spin rotations along one axis). This symmetry is never exact in real magnets due to the presence of anisotropic interactions such as the classical dipole-dipole coupling or the effective couplings induced by the spin-orbit interaction in the presence of lattice anisotropy. Thus, the number of bosons is subject to small fluctuations. Consequently, the thermodynamic properties of the quantum magnet are well described by a number-conserving theory if the temperature is well above the magnitude of the uniaxial symmetry-breaking terms. However, nonequilibrium properties of BEC states such as supercurrents will occur only on very short time scales. What we strive for is a system in which the uniaxial symmetrybreaking interactions occur at significantly lower temperatures than the maximum critical temperature for the onset of the BEC state.

Besides the quantum magnets reviewed in this paper, there are a few other examples of BECs in solids. Several groups have studied condensation of excited magnons into metastable states, which are predicted to form a BEC over the time scale of metastability. These systems include optically pumped yttrium iron garnet films (Demokritov et al., 2006), and coherently precessing spins in NMR experiments on $\mathrm{CsMnF}_{3}$ (Bunkov et al., 2011), ${ }^{3} \mathrm{He}-A$ (Bunkov and Volovik, 2010), and ${ }^{3} \mathrm{He}-B$ (Bunkov and Volovik, 2007). We should clarify the differences between the BEC of magnons in these four examples, and the BEC in quantum magnets reviewed in this paper. In BECs of magnons, the BEC is metastable since magnons are inherently excitations, whereas in quantum magnets the BEC occurs in the thermodynamic ground state of the system. The other qualitative difference is that in BECs of magnons the condensing particles are magnons, whereas in quantum magnets the condensing bosons are obtained by mapping the long-range magnetically ordered ground state onto a lattice of bosons. Magnons in quantum magnets are actually excitations out of the condensed ground state of the spins. This issue is confused by the fact that authors of quantum magnet BEC papers sometimes use the terminology "BEC of magnons."

There has also been a lot of work on BECs of excitons, which are electron-hole pairs excited across a gap that form metastable bound states (Blatt, Böer, and Brandt, 1962; Keldysh and Kopaev, 1964; Halperin and Rice, 1968). Most early experiments investigated metastable excitons produced, e.g., by optical pumping in semiconductors, where the goal is to extend the lifetime of the excitons beyond the thermalization time of the condensate. These exciton condensates have been explored experimentally and theoretically 
in many confined geometries including coupled quantum wells (Butov et al., 1994; Butov et al., 2002; Mani et al., 2002; Snoke, 2002) and semiconductor microcavities (Snoke, 2002; Deng, Haug, and Yamamoto, 2010). Excitonic condensates have also been proposed to occur in equilibrium in bilayer quantum Hall systems (Eisenstein and MacDonald, 2004; Nandi et al., 2012). Here, the coupling between electrons and holes, or electrons and electrons occupying Landau levels in adjacent 2D layers, produces exciton bound states with very long lifetimes. These produce "Coulomb drag," where a current in one layer produces a current in the other. Excitonic condensates have also been predicted to occur in thin films of topological insulators (Seradjeh, Moore, and Franz, 2009). Finally, condensation and/or crystallization of excitons is also proposed as one explanation for pressureinduced insulating behavior in certain $4 d$ chalcogenides (Mott, 1961; Wachter, Bucher, and Malar, 2004).

The primary focus of this paper is BEC in quantum magnets, and we review the large amount of experimental and theoretical work that has taken place during the last two decades. In the following sections we describe the mapping between spins and bosons in detail and the requirements for uniaxial symmetry and number conservation. We then cover the different analytical and numerical approaches for calculating the properties of bosonic quantum magnets and describe how these apply to real systems. We go on to discuss the most important experimental probes and the experimental evidence for BEC-like quantum phase transitions, and we conclude with an overview of the field of magnetic realizations of bosonic gases and the rich variety of bosonic phases that can be studied in the future.

Finally we apologize to the many whose work we were not able to treat in the depth it deserved in this review.

\section{THEORY}

\section{A. Mappings between spin and itinerant-particle systems}

The connection between magnetic spin systems and gases of itinerant particles became clear after the introduction of two mathematical transformations several decades ago. The first was introduced by Jordan and Wigner (1928) for one-dimensional systems. It consists of a nonlocal mapping between $S=1 / 2$ spin operators and creation and annihilation operators for spinless fermions,

$$
S_{j}^{+}=a_{j}^{\dagger} e^{i \pi \sum_{k<j} n_{k}^{a}}, \quad S_{j}^{-}=a_{j} e^{i \pi \sum_{k<j} n_{k}^{a}}, \quad S_{j}^{z}=n_{j}^{a}-\frac{1}{2},
$$

where $a_{j}^{\dagger}\left(a_{j}\right)$ is the creation (annihilation) operator of a spinless fermion in the site or orbital $j$ and $n_{j}^{a}=a_{j}^{\dagger} a_{j}$ is the occupation number. The Jordan-Wigner transformation (1) is not only an efficient tool for diagonalizing some onedimensional spin Hamiltonians such as the $X Y$ spin-1/2 chain or the quantum Ising model. This transformation is also helpful for understanding the nature of the ground state and typical low-energy excitations of one-dimensional systems. Furthermore, it shows that the particle statistics (fermions or bosons) can be absorbed into few-body interactions when the system is one dimensional and the bare interactions are short ranged. These are examples of the remarkable insight that is provided by mappings between different physical systems.

Nearly two decades after Jordan and Wigner introduced their mapping between $S=1 / 2$ spins and spinless fermions, Matsubara and Matsuda (1956) presented an even simpler mapping between $S=1 / 2$ spin operators and hard-core bosons,

$$
S_{j}^{+}=b_{j}^{\dagger}, \quad S_{j}^{-}=b_{j}, \quad S_{j}^{z}=b_{j}^{\dagger} b_{j}-\frac{1}{2} .
$$

Here $b^{\dagger}$ and $b$ are the creation and annihilation operators of the hard-core bosons. In contrast to the Jordan-Wigner mapping, the Matsubara-Matsuda transformation is local and, consequently, it does not depend on the dimension of the system under consideration. This is so because spin operators on different sites satisfy bosonic exchange statistics: $\left[S_{j}^{\mu}, S_{k}^{\nu}\right]=0$ for $j \neq k$ and $\mu, \nu=\{x, y, z\}$. This simple mapping between localized magnetic moments and a gas of itinerant hard-core bosons establishes a formal analogy between seemingly unrelated phenomena, i.e., different types of magnetic orderings are mapped into different ordered phases of bosonic gases.

The Jordan-Wigner transformation is useful for solving $\mathrm{U}(1)$ invariant one-dimensional spin models because spinless fermions provide a natural realization of two-level systems. In other words, there is no need to introduce the hard-core constraint on occupancy, because the fermionic statistics guarantees that the occupation number of each site cannot be higher than 1 . Consequently, we start by considering the fermionic version of a prototypical one-dimensional spin Hamiltonian. After describing the advantages of using a fermionic language for treating one-dimensional U(1) invariant spin models, we apply the Matsubara-Matsuda mapping to the same spin model in spatial dimension $d \geq 2$.

A rather ubiquitous one-dimensional spin model consists of a chain of $S=1 / 2$ moments coupled by an antiferromagnetic exchange interaction $J$, with uniaxial exchange anisotropy determined by the parameter $\gamma$. The corresponding spin Hamiltonian is

$$
\mathcal{H}_{X X Z}=J \sum_{j}\left(S_{j}^{x} S_{j+1}^{x}+S_{j}^{y} S_{j+1}^{y}+\gamma S_{j}^{z} S_{j+1}^{z}\right)-g_{z z} \mu_{B} H \sum_{j} S_{j}^{z},
$$

where $J>0$ (antiferromagnetic exchange), $g_{z z}$ is the gyromagnetic factor along the $z$ axis, and $\mu_{B}$ is the Bohr magneton. We also included the Zeeman coupling to an external magnetic field $H$ applied along the symmetry or $z$ axis. After applying the Jordan-Wigner transformation to (3), we obtain the following tight-binding Hamiltonian for spinless fermions with nearest-neighbor repulsive interactions:

$$
\begin{aligned}
\mathcal{H}_{X X Z}= & \frac{J}{2} \sum_{j}\left(a_{j}^{\dagger} a_{j+1}+a_{j+1}^{\dagger} a_{j}\right) \\
& +\gamma J \sum_{j}\left(n_{j}^{a}-1 / 2\right)\left(n_{j+1}^{a}-1 / 2\right)-\mu \sum_{j} n_{j}^{a},
\end{aligned}
$$


where the chemical potential $\mu=g_{z z} \mu_{B} H$ is proportional to the magnetic field. From Eq. (4) we can see that $\mathcal{H}_{X X Z}$ conserves the total number of particles, i.e., it commutes with $N=\sum_{j} n_{j}^{a}$. In other words, $\mathcal{H}_{X X Z}$ is invariant under the global $\mathrm{U}(1)$ transformation, $\tilde{c}_{j}^{\dagger}=e^{i \varphi} c_{j}^{\dagger}$. According to Eq. (1), the Jordan-Wigner transformation maps the total number of fermions into the total magnetization plus a constant. Therefore, conservation of the total number of fermions corresponds to conservation of the total magnetization along the $z$ axis. The corresponding $\mathrm{U}(1)$ symmetry is simply a global rotation by an angle $\phi$ along the $z$ axis: $\tilde{S}_{j}^{+}=e^{i \phi} S_{j}^{+}$.

The total number of particles is controlled by the chemical potential $\mu=g_{z z} \mu_{B} H$. Therefore, the ground state is populated with particles with increasing magnetic field. The maximum number of particles is one fermion per site and it is reached at the saturation field $H_{\mathrm{sat}}=J(1+\gamma) / g_{z z} \mu_{B}$. Since there is only one state with one fermion per site, the ground state remains invariant for $H \geq H_{\text {sat }}$. The spectrum of single-particle excitations is obtained by removing a single fermion (creating a single hole) from the fully occupied ground state. The exact dispersion relation is obtained by solving the corresponding single-particle problem which is diagonal in momentum space because of the translational invariance of $\mathcal{H}_{X X Z}$,

$$
\omega_{k}=J+J \cos k+g \mu_{B}\left(H-H_{\text {sat }}\right) .
$$

Here and in the rest of the review we use the lattice parameter as the unit of length and $\hbar=1$. This dispersion relation has a minimum at the antiferromagnetic wave vector $k=Q=\pi$ and a gap that changes linearly with the magnetic field. The gap closes at the saturation field $H_{\text {sat }}$ and the corresponding gapless mode at $k=Q$ leads to a divergent susceptibility at the antiferromagnetic wave vector. In the long-wavelength limit, $k=Q+q$ with $q \ll 1$, the dispersion relation at $H=H_{\text {sat }}$ can be approximated by $\omega_{q} \simeq J q^{2} / 2$. This is the dispersion relation of a free fermion with mass $m^{*}=1 / J$. We will see in the next section that the field-induced quantum critical point at $H=H_{\text {sat }}$ is indeed a free-fermion fixed point, i.e., interaction terms like the repulsion between nearest neighbors that appears in $\mathcal{H}_{\mathrm{XXz}}$ are irrelevant in the longwavelength limit.

The dynamical exponent $z$ or scaling dimension of the inverse of the correlation length along the imaginary time direction $1 / \xi_{\tau}$ (Sachdev, 1999) determines the power-law exponent of the single-particle dispersion at the QCP, $\omega_{q} \propto q^{z}$. Since $\omega_{q} \propto q^{2}$ at $H=H_{\text {sat }}$, the dynamical exponent of this field-induced QCP is $z=2$. In general, the quasiparticle dispersion is quadratic at the saturation field because the magnetic field couples to a conserved quantity that is the total magnetization along the $z$ axis $M_{z}=\sum_{j} S_{j}^{z}$. Regardless of details of the interactions or the system dimensionality, the single-particle excitations above the saturation field correspond to eigenstates with a single spin flip, $M_{z}=N / 2-1$, relative to the fully polarized ground state. The restricted action of the Hamiltonian on this invariant subspace leads to an effective single-particle problem that is exactly diagonalized in the basis of well-defined momentum if the system under consideration is translationallly invariant. Since these excited states have $M_{z}=N / 2-1$ and the ground state has $M_{z}=N / 2$, the energy gap $\Delta$ for exciting the lowest-energy quasiparticle state must increase linearly with the applied magnetic field, $\Delta=\omega_{\mathbf{Q}}=g_{z z} \mu_{B}\left(H-H_{\text {sat }}\right)$. Since the singleparticle dispersion relation $\omega_{\mathbf{k}}$ is an analytic function of $\mathbf{k}$, the expansion around its minimum value at $\mathbf{k}=\mathbf{Q}$ will lead in general to a quadratic dispersion or $z=2$. Dispersions with higher even powers like $\omega_{\mathbf{k}} \simeq \omega_{\mathbf{Q}}+c(\mathbf{k}-\mathbf{Q})^{4}$ would require fine-tuning of the Hamiltonian parameters. The conservation of $M_{z}$ implies that $\omega_{\mathbf{k}}-\omega_{\mathbf{Q}}$ does not depend on $H$ for $H \geq H_{\text {sat }}$ (the shape of $\omega_{\mathbf{k}}$ does not change). Therefore, the dispersion remains quadratic and the mass remains constant for any $H \geq H_{\text {sat, }}$, i.e., $z=2$, except for very particular cases in which the interactions are tuned to produce higher even values of $z$. For instance, if we add a next-nearest-neighbor exchange interaction $J^{\prime} \leq J / 2$ to $\mathcal{H}_{\mathrm{XXZ}}$, the single-particle dispersion becomes $\omega_{k}=J+J \cos k+J^{\prime} \cos 2 k+g \mu_{B}\left(H-H_{\text {sat }}\right)$ and the expansion around its minimum at $Q=\pi$ gives $\omega_{k}=\omega_{Q}+\left(J / 2-J^{\prime}\right)(k-Q)^{2}+\left(J / 24+2 J^{\prime} / 3\right)(k-Q)^{4}$. This implies that $z$ becomes equal to 4 at the fine-tuned commensurate to incommensurate transition point $J^{\prime}=J / 2$.

When the quantum phase transition at $H=H_{\text {sat }}$ is of second order, the corresponding critical point is called Bose-Einstein condensate QCP (BEC QCP). In the next section we will discuss in detail the characteristics of this QCP. For the moment we just observe that the effective dimension of this QCP is $D=d+z=d+2$, where $d$ is the spatial dimensionality of the system under consideration. We will see later on that for certain frustrated systems the value of $d$ can be lower than their actual spatial dimensionality (Sebastian, Harrison et al., 2006). This phenomenon is called dimensional reduction but for the moment we will not worry about this effect. Since $D=d+2$, the upper critical dimension of the BEC QCP is $d_{c}=2$. This means that a mean-field bosonic description should be adequate in $d \geq 2$ except for logarithmic corrections that exist for $d=2$.

The next step is to find the best low-energy effective theory for a mean-field description for the BEC QCP in $d \geq 2$. Here is where the Matsubara-Matsuda transformation (2) plays an important role. As we will see in a moment, this transformation maps the magnetic problem near the saturation field into the problem of a dilute gas of interacting bosons. Fortunately, this problem can be solved in a controlled way in $d \geq 2$ by using diagrammatic techniques (perturbation theory). The small or "control" parameter of the expansion is the ratio between the scattering length and the average distance between bosons (lattice gas parameter). This is enough motivation for applying the Matsubara-Matsuda transformation (2) to the $d$-dimensional versions of $\mathcal{H}_{x x z}$. The natural extension of Eq. (3) to $d \geq 2$ corresponds to the case of a hypercubic $d$-dimensional lattice with the same type of nearest-neighbor interactions,

$$
\begin{aligned}
\mathcal{H}_{X X Z}= & J \sum_{\mathbf{r}, \nu}\left(S_{\mathbf{r}}^{x} S_{\mathbf{r}+\mathbf{e}_{\nu}}^{x}+S_{\mathbf{r}}^{y} S_{\mathbf{r}+\mathbf{e}_{\nu}}^{y}+\gamma S_{\mathbf{r}}^{z} S_{\mathbf{r}+\mathbf{e}_{\nu}}^{z}\right) \\
& -g_{z z} \mu_{B} H \sum_{\mathbf{r}} S_{\mathbf{r}}^{z}
\end{aligned}
$$

After applying the Matsubara-Matsuda transformation (2) to (6) we obtain 


$$
\begin{aligned}
\mathcal{H}_{X X Z}= & \frac{J}{2} \sum_{\mathbf{r}, \nu}\left(b_{\mathbf{r}}^{\dagger} b_{\mathbf{r}+\mathbf{e}_{\nu}}+b_{\mathbf{r}+\mathbf{e}_{\nu}}^{\dagger} b_{\mathbf{r}}\right) \\
& +\gamma J \sum_{\mathbf{r}}\left(n_{\mathbf{r}}^{b}-1 / 2\right)\left(n_{\mathbf{r}+\mathbf{e}_{\nu}}^{b}-1 / 2\right)-\mu \sum_{\mathbf{r}} n_{\mathbf{r}}^{b} .
\end{aligned}
$$

$\mathcal{H}_{\mathrm{XXZ}}$ has particle-hole symmetry, i.e., it is invariant under the transformation $b_{\mathbf{r}}^{\dagger} \rightarrow b_{\mathbf{r}}$ and $\mu \rightarrow-\mu$. This invariance is a direct consequence of the time-reversal symmetry $\mathcal{H}_{X X Z}$ in the original spin language: $\mathbf{S}_{j} \rightarrow-\mathbf{S}_{j}$ and $H \rightarrow-H$. Timereversal symmetry implies that the system has two saturation fields $\pm H_{\text {sat }}$ with $g_{z z} \mu_{B} H_{\text {sat }}=d J(1+\gamma)$ [see Fig. 1(a)]. The ground state of $\mathcal{H}_{\mathrm{XXZ}}$ for $H \leq-H_{\text {sat }}$ consists of the fully polarized spin state in the down direction $|\downarrow\rangle$, with $S_{j}^{z}|\downarrow\rangle=-(1 / 2)|\downarrow\rangle$. Conversely, the ground state of $\mathcal{H}_{X X Z}$ for $H \geq H_{\text {sat }}$ consists of the fully polarized spin state in the up direction $|\uparrow\rangle$, with $S_{j}^{z}|\uparrow\rangle=(1 / 2)|\uparrow\rangle$. In the particle language we have that the fully polarized states correspond to the empty state and the state with one hard-core boson in each site: $|\downarrow\rangle \leftrightarrow|0\rangle$ and $|\uparrow\rangle \leftrightarrow \prod_{\mathbf{r}} b_{\mathbf{r}}^{\dagger}|0\rangle$. Since there is particle-hole symmetry, the BEC QCPs at $H= \pm H_{\text {sat }}$ are identical and it is enough to analyze only one of them. We consider the case in which $H$ is close to $-H_{\text {sat }}$. By rewriting Eq. (4) in momentum space we obtain

$$
\mathcal{H}_{X X Z}=\sum_{\mathbf{k}}\left(\omega_{\mathbf{k}}-\tilde{\mu}\right) \hat{b}_{\mathbf{k}}^{\dagger} \hat{b}_{\mathbf{k}}+\frac{1}{2 L} \sum_{\mathbf{k}, \mathbf{k}^{\prime}, \mathbf{q}} V_{\mathbf{q}} \hat{b}_{\mathbf{k}+\mathbf{q}}^{\dagger} \hat{b}_{\mathbf{k}^{\prime}-\mathbf{q}}^{\dagger} \hat{b}_{\mathbf{k}} \hat{b}_{\mathbf{k}^{\prime}}
$$

where

$$
\begin{aligned}
& \hat{b}_{\mathbf{k}}^{\dagger}=\frac{1}{\sqrt{L}} \sum_{\mathbf{r}} e^{i \mathbf{k} \cdot \mathbf{r}} b_{\mathbf{r}}^{\dagger}, \quad \omega_{\mathbf{k}}=J \sum_{\nu}\left(1+\cos k_{\nu}\right), \\
& \tilde{\mu}=g_{z z} \mu_{B}\left(H+H_{\mathrm{sat}}\right), V_{\mathbf{q}}=U+2 \gamma \mathrm{J} \sum_{\nu} \cos k_{\nu},
\end{aligned}
$$

and $L$ is the total number of lattice sites. The on-site repulsion $U$ has been introduced to enforce the hard-core constraint by taking the limit $U \rightarrow \infty$ (Friedberg, Lee, and Ren, 1993). Therefore, the bosonic operators $\hat{b}_{\mathbf{k}}^{\dagger}$ create canonical bosons (they satisfy the usual bosonic commutation relations) with well-defined momentum $\mathbf{k}$. We note that $\omega_{\mathbf{k}} \simeq J k^{2} / 2$ for $k \ll 1$ and $\tilde{\mu}=0$ for $H=-H_{\text {sat }}$. As mentioned, a mean-field treatment of the interaction term of Eq. (8) is valid for $d \geq 2$. In the noncondensed phase, the mean-field decoupling of the interaction term leads only to a renormalization of the chemical potential, $\tilde{\mu} \rightarrow \tilde{\mu}-2 \rho v\left(\rho=\left\langle b_{j}^{\dagger} b_{j}\right\rangle\right.$ is the density of bosons, and $v$ is the effective interaction in the longwavelength limit). Therefore, the BEC QCP in $d \geq 2$ corresponds to a free-boson fixed point.
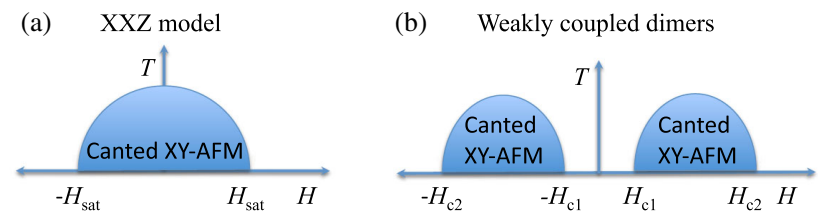

FIG. 1 (color online). $\quad(H, T)$ phase diagrams of (a) $S=1 / 2 X X Z$ and (b) weakly coupled dimer models.
The Matsubara-Matsuda transformation can be generalized for higher spin values (Batista and Ortiz, 2004). The crucial point is that spin degrees of freedom commute on different sites. Consequently, they can always be represented by bosonic particles. For instance, one of the spin states $\mid S^{z}=$ $-S\rangle$ can be mapped into the empty state of bosons $|\varnothing\rangle$, while the other $2 S$ spin states can be mapped into the states that have different numbers of bosons up to $2 S$. Therefore, the hard-core constraint $\left(b_{\mathbf{r}^{\prime}}^{\dagger}\right)^{2}=0$ is generalized to $\left(b_{\mathbf{r}^{\prime}}^{\dagger}\right)^{2 S+1}=0$. In this way, one can extend the previous approach to any spin system. As we see in the next sections, there are cases in which the unit cell contains more than one site and it is more convenient to describe the system in terms of local degrees of freedom that include more than one irreducible spin representation. A simple example is a dimer of two $S=1 / 2$ spins that can be in a singlet or in one of the three triplet states. The Matsubara-Matsuda transformation can also be applied to this case, which is particularly relevant for many quantum paramagnets that have been studied during the last two decades.

The spin-boson mapping allows us to compare the types of Hamiltonians that are relevant for both types of systems. Although the minimal Hamiltonians for describing the $S=$ $1 / 2$ magnetic systems that appear in nature are apparently very similar to the ones that describe gases of strongly repulsive bosonic atoms on a lattice, there are also important differences in the general symmetry properties of both types of Hamiltonians. For instance, we know that the total number of particles is a conserved quantity for atomic gases. This conservation is associated with a global U(1) symmetry of the corresponding Hamiltonians. As we will see below, spin Hamiltonians that possess this U(1) symmetry are only approximated models for a class of quantum magnets. Although the approximated model can provide an excellent description of certain spin systems down to very low temperatures, we will see that terms that break any continuous symmetry are always present and become relevant at low enough temperatures. It is for this reason that the notion of Bose-Einstein condensation applied to spin systems is always an approximated concept. Like many other instances in physics, this approximated notion has been useful for analyzing and understanding different physical properties of most of the quantum magnets that we describe in this review.

\section{B. Uniaxial symmetry breaking and superfluid currents}

We have seen in the previous section that any spin Hamiltonian can be described as a gas of itinerant bosons by using exact transformations that map the spin operators into creation and annihilation operators of bosons that satisfy some constraint. We have also seen that this mathematical fact can be exploited for solving spin Hamiltonians. Since the transition to the BEC state corresponds to a spontaneous breaking of the continuous U(1) symmetry that preserves the number of particles, the spin Hamiltonian must contain this $\mathrm{U}(1)$ symmetry to exhibit a spontaneous transition to a BEC state. As discussed, this requirement is fulfilled by magnets with continuous uniaxial symmetry. The purpose of this section is to emphasize that real magnets always contain interactions that break this U(1) symmetry, and thus U(1)invariant Hamiltonians are very good approximations for only 
a subset of these systems. The "degree of U(1) symmetry" is the most important factor that constrains the number of real quantum magnets that can be described in terms of BEC. It is incumbent then on the researcher to understand how large the broken uniaxial symmetry terms are in comparison with the ordering temperature of the system. Here we explore different ways in which the uniaxial symmetry can be broken.

In general, the exchange interaction between two magnetic moments $\mathbf{S}_{\mathbf{r}}$ and $\mathbf{S}_{\mathbf{r}+\mathbf{e}_{\nu}}$ has a Heisenberg-like contribution $J \mathbf{S}_{\mathbf{r}} \cdot \mathbf{S}_{\mathbf{r}+\mathbf{e}_{\nu}}$ that is isotropic, i.e., invariant under any global spin rotation. However, there are also anisotropic contributions that arise from the spin-orbit interaction that transfers the lattice anisotropy into the spin variables. The most general exchange interaction between two moments has the form $\sum_{i, j} S_{\mathbf{r}}^{i} T^{i j} S_{\mathbf{r}+\mathbf{e}_{\nu}}^{j}$ (Moriya, 1960; Sebastian, Tanedo et al., 2006), where

$$
\begin{aligned}
\mathbf{T} & =\frac{1}{3} \operatorname{Tr}(\mathbf{T}) \mathbf{I}+\mathbf{T}_{\mathrm{as}}+\mathbf{T}_{\mathrm{sm}}, \\
\mathbf{T}_{\mathrm{as}} & =\frac{1}{2}\left(\mathbf{T}-\mathbf{T}^{\dagger}\right), \\
\mathbf{T}_{\mathrm{sm}} & =\frac{1}{2}\left(\mathbf{T}+\mathbf{T}^{\dagger}\right) .
\end{aligned}
$$

Here $\mathbf{I}$ is the identity and the first term leads to the usual isotropic exchange coupling $J=\operatorname{Tr}(\mathbf{T}) / 3 . \mathbf{T}_{\text {as }}$ is an antisymmetric tensor that describes the Dzyaloshinskii-Moriya (DM) interaction $\mathbf{D} \cdot \mathbf{S}_{\mathbf{r}} \times \mathbf{S}_{\mathbf{r}+\mathbf{e}_{\nu}}$, where $D^{\eta}=\sum_{\mu, \nu} \epsilon^{\eta \mu \nu} T_{\text {as }}^{\mu \nu}$ are the components of the DM vector, and $\epsilon_{\eta \mu \nu}$ is the Levi-Cività symbol (Moriya, 1960). The last term $\mathbf{T}_{\mathrm{sm}}$ contains the socalled symmetric exchange anisotropy and has contributions from the classical dipole-dipole interaction between magnetic moments. Dipole-dipole interactions always exist and the axis of uniaxial symmetry is the one that connects the two moments. Since this axis is different for bonds that are oriented along different directions, it is not possible to have a real $2 \mathrm{D}$ or $3 \mathrm{D}$ quantum magnet with continuous uniaxial symmetry along any axis. However, for the small spins ( $S=1 / 2$ or 1 ) found in most BEC compounds, and for typical lattice spacings of 10 to $3 \AA$, the dipole-dipole interactions are of the order of $10-100 \mathrm{mK}$. Thus, these interactions are much smaller than typical BEC transition temperatures, which can range from 1 to $100 \mathrm{~K}$.

In addition to anisotropy in the interaction between two spins, there are also single-spin terms that can break uniaxial anisotropy (single-ion anisotropy). For example, in a system with cubic anisotropy, the spins could prefer to lie along principal axes, resulting in a clock like model rather than $X Y$ symmetry.

Careful measurements of all the U(1) symmetry-breaking terms were performed in several compounds, and these showed that the symmetry-breaking terms are small enough to provide a clean experimental window where the physics is well described by a number-conserving theory (Nikuni et al., 2000; Sebastian, Harrison et al., 2006; Batista et al., 2007; Schmalian and Batista, 2008). In addition, by understanding the BEC phenomenology in idealized U(1)-invariant magnets we can treat $\mathrm{U}(1)$ symmetry-breaking terms perturbatively (Oshikawa and Affleck, 1997; Kolezhuk et al., 2004; Misguich and Oshikawa, 2004; Sirker, Weiße, and Sushkov, 2004; Matsumoto and Sigrist, 2005; Orignac and
Citro, 2005; Sebastian, Tanedo et al., 2006; Miyahara et al., 2007).

Finally, it is important to mention that the U(1) symmetry plays a fundamental role in the superfluid response of bosonic systems. The conservation of the number of particles can be expressed via a continuity equation,

$$
\frac{\partial \rho(\mathbf{r})}{\partial t}+\nabla \cdot \mathbf{j}(\mathbf{r})=0
$$

where $\rho(\mathbf{r})$ and $\mathbf{j}(\mathbf{r})$ are the particle and current densities at the point $\mathbf{r}$. This equation implies that the response of the gas of bosons to a chemical potential gradient $\nabla \mu$ is a current: because particles cannot be created or annihilated, the only response to $\nabla \mu$ is a flow of particles toward the region with higher values of $\mu$. In particular, this current becomes superfluid in the condensate phase, i.e., it flows without decaying in a ring configuration unless a vortex moves across the ring. Since the probability of such an event is exponentially small in the thickness of the ring, the superfluid current of a BEC of atoms can persist for arbitrarily long times. The lack of continuous symmetry for magnetic systems implies that there is no continuity equation for the magnetization. In other words, the righthand side of the magnetic counterpart of Eq. (11) is different from zero and proportional to the inverse of the relaxation time, $\tau_{m}$ of the magnetization,

$$
\frac{\partial m(\mathbf{r})}{\partial t}+\nabla \cdot \mathbf{j}_{m}(\mathbf{r}) \propto \frac{1}{\tau_{m}}
$$

Here $m(\mathbf{r})$ and $\mathbf{j}_{m}(\mathbf{r})$ are the densities of magnetization (along the field direction) and the magnetization current at the point r. Equation (12) shows that magnetization is not conserved and magnetization currents decay with a time constant $\tau_{m}$. Consequently, the anisotropy terms that are responsible for the finite value of $\tau_{m}$ preclude a long-term superfluid response of the magnetic system.

\section{Quantum criticality}

\section{BEC quantum critical points}

In the previous sections we investigated the differences and commonalities between quantum magnets and gases of bosonic atoms. The purpose of this section is to characterize the generic quantum critical point that connects the $X Y$-like antiferromagnet with the gapped paramagnet when the external magnetic field is varied. In the language that is more common for gases of atoms, this corresponds to the quantum phase transition between the Mott insulator and the BEC phases that is induced by a change in the chemical potential $\mu$. In Sec. II.A we analyzed a particular realization of this type of transition in systems that are antiferromagnetic in the absence of the external field. In these cases the transition occurs at the critical field required for saturating the uniform magnetization. An alternative realization of the BEC QCP is obtained in materials that are paramagnetic in absence of an external field. In these so-called quantum paramagnets [see Fig. 1(b)], there is a zero-field gap between a nonmagnetic ground state and a magnetic excited state, leading to a ground state with no 
magnetic order. In applied magnetic fields, this gap can be closed, leading to a QCP into the $X Y$-AFM state that takes place at a BEC QCP. Most zero-field quantum paramagnets belong to one of three classes. The first class consists of materials such as $\mathrm{TlCuCl}_{3}$ or $\mathrm{BaCuSi}_{2} \mathrm{O}_{6}$, where the spin gap is caused by strong dimerization of the magnetic ions (Nikuni et al., 2000; Jaime et al., 2004). The second class consists of materials like $\mathrm{NiCl}_{2}-4 \mathrm{SC}\left(\mathrm{NH}_{2}\right)_{2}$ (DTN), where the gap is created by a dominant single-ion anisotropy that quenches the moment of each magnetic ion (Zapf et al., 2006). The main difference is that materials in the first class are isotropic to a good approximation before the external field is applied, while the materials in the second class have only uniaxial symmetry. A third but less frequent possibility corresponds to the case of quasi-one-dimensional systems that can have a gapped paramagnetic ground state even if they are isotropic and contain one spin per unit cell. The Haldane phase of integer spin antiferromagnetic chains is the prototypical example (Haldane, 1983). There are many other ways to create quantum paramagnetism, and some of these are listed in the later experimental sections. Here we analyze two of the most common, namely, dimerized systems and compounds with strong single-ion anisotropy.

Several quantum paramagnets are quasi-one- or quasi-twodimensional. Therefore, before considering the more realistic situation of field-induced antiferromagnetism in systems with spatial dimension $d=3$, it is instructive to analyze the $d=1$ and $d=2$ limits. There are materials in which the lowerdimensional behavior (critical exponents of the $d=1$ or $d=2$ QCPs) can be observed over a quite large window of temperatures. Note that the critical scaling exponent $\nu$, which relates the correlation length to the driving parameter of the phase transition, $\xi \sim\left|H-H_{c}\right|^{-\nu}$ is equal to $1 / 2$ regardless of the spatial dimensionality. This unusual property was originally derived by Sachdev (1994) and arises from a symmetry property: the driving parameter $\mu$ couples to a conserved quantity that is the total number of particles. We have seen in Sec. II.A that this property leads to a dynamical exponent $z=2$. It also leads to a gap that closes linearly in the driving parameter $\mu$ because the ground state and the first excitation have zero and one particle, respectively. Therefore, if $\xi$ is the magnetic correlation length that diverges at the BEC QCP, the following scaling relations hold for $d \leq d_{c}=2$ :

$$
\Delta \propto \mu \propto \frac{1}{\xi^{1 / \nu}} \quad \text { and } \quad \Delta \propto \frac{1}{\xi^{z}}
$$

implying that

$$
\nu=1 / z=1 / 2 \text {. }
$$

This result is still correct for $d>d_{c}$ because $\nu=1 / 2$ for the mean-field theory that is applicable to those cases.

We start by considering the $d=1$ case. We have seen in Sec. II.A that any $d=1$ spin Hamiltonian can be expressed in terms of fermionic operators by means of a Jordan-Wigner transformation. In addition, for the particular model that we considered in that section, we obtained the result that the fieldinduced QCP, where the gap closes, is described by a freefermion model. As we argue in this section, this is a generic behavior. It is then natural to use a fermionic language for treating the $d=1$ case of interest.

The long-wavelength limit can be obtained by reducing the lattice parameter $a \rightarrow 0$. In this continuum limit, the partition function $Z=\operatorname{Tr} e^{-\beta \mathcal{H}}$ can be expressed as a path integral,

$$
Z=\int \mathcal{D} \psi \mathcal{D} \psi^{\dagger} e^{-\int_{0}^{\beta \hbar} \mathcal{L} d \tau d x},
$$

where the functional integral is over complex Grassmann fields, $\psi(x, \tau)$ and $\psi^{\dagger}(x, \tau)$, and the Lagrangian density $\mathcal{L}=$ $\mathcal{L}_{0}+\mathcal{L}_{I}$ can be obtained as a gradient expansion in the Grassmann fields. $\mathcal{L}_{0}$ contains the quadratic contributions in the Grassmann fields and describes a nonrelativistic free-fermion theory,

$$
\begin{aligned}
\mathcal{L}_{0}= & \psi^{\dagger}(x, \tau) \partial_{\tau} \psi(x, \tau)-\frac{1}{2 m^{*}} \psi^{\dagger}(x, \tau) \partial_{x}^{2} \psi(x, \tau) \\
& +\mu \psi^{\dagger}(x, \tau) \psi(x, \tau) .
\end{aligned}
$$

For $\mu=0$ and $T=0$, the action $\mathcal{S}=\int_{0}^{\beta} \mathcal{L} d \tau d x$ becomes invariant under the scale transformation,

$$
x^{\prime}=x e^{-l}, \quad \tau^{\prime}=\tau e^{-z l}, \quad \psi^{\prime}=\psi e^{l / 2},
$$

where the dynamical exponent is $z=2$ because the first term of $\mathcal{L}_{0}$ contains only one time derivative, while the second term contains two spatial derivatives. Indeed, by expressing $\mathcal{L}_{0}$ in terms of the Fourier components of the Grassmann fields $\tilde{\psi}^{\dagger}(q, \omega)$ and $\tilde{\psi}(q, \omega)$, we obtain the dispersion relation as

$$
\omega_{q}=\frac{q^{2}}{2 m^{*}}
$$

According to Eqs. (17), the scaling dimension of the chemical potential or driving parameter is $1 / \nu=\operatorname{dim}[\mu]=z=2$ in agreement with Eq. (14).

The next step is to compute the scaling dimension of the interacting terms of $\mathcal{L}$. The leading contribution in the gradient expansion is

$$
\mathcal{L}_{I}=u \psi^{\dagger}(x, \tau) \partial_{x} \psi^{\dagger}(x, \tau) \psi(x, \tau) \partial_{x} \psi(x, \tau) .
$$

Note that the Pauli exclusion principle implies that the interaction term must include gradients. The lattice counterpart of this observation is that the density-density interactions between fermions are always off site because we cannot put two spinless fermions on the same site. Consequently, by using Eqs. (17) we obtain the scaling dimension of $u$ as $\operatorname{dim}[u]=z+1-4=-1$. In other words, the interaction terms are irrelevant for the free-fermion fixed point described by $\mathcal{L}_{0}$.

Since the field-induced QCP in $d=1$ is described by a freefermion theory, it is easy to compute the exponents of the different thermodynamic properties as function of the relevant parameters $\mu$ and $T$. In particular, at $T=0$, the fermion density (or magnetization density in the spin language) as a function of $\mu$ is given by 


$$
\rho=\frac{1}{\pi} \int_{0}^{q_{f}} d q=\frac{q_{f}}{\pi}=\frac{1}{\pi} \sqrt{2 m^{*} \mu},
$$

where $q_{f}$ is the Fermi wave vector, i.e., $\omega\left(q_{f}\right)=\mu$. This result implies that the magnetization of one-dimensional magnets increases from zero with an infinite slope at the critical field $H_{c}: m(T=0, H) \propto \sqrt{H-H_{c}}$. This anomalous increase in the magnetization provides one experimental check for detecting quasi-one-dimensional magnets. As we see below, the behavior of $m(T=0, H)$ is qualitatively different for higher-dimensional systems. On the other hand, the temperature dependence at $H=H_{c}$ is again obtained by integrating the Fermi function, $m\left(T, H=H_{c}\right)=$ $\int f\left(\omega_{q} / k_{B} T\right) d q \propto T^{1 / 2}$. The exponent $1 / 2$ results from the fact that $d q$ scales like $T^{1 / z}$. The same exponent is obtained for the specific heat: $C\left(T, H=H_{c}\right) \propto T^{1 / 2}$.

Given the simplicity of the $d=1$ fixed point, it is possible to accurately compute any physical property in the neighborhood of the field-induced QCP. In particular, the pioneering work of Giamarchi and Tsvelik (1999) contains a derivation of the NMR relaxation time- $T_{1}, 1 / T_{1} \propto T \lim _{\omega \rightarrow 0} \chi_{\text {loc }}^{\prime \prime}(\omega) /$ $\omega \propto 1 / T^{1 / 2}$, where $\chi_{\text {loc }}^{\prime \prime}(\omega)$ is the imaginary part of the local magnetic susceptibility in the direction perpendicular to the applied field. The implicit assumption is that the local susceptibility near the QCP is dominated by the contribution from the transverse part of the staggered susceptibility. Once again, this result can be obtained by computing the scaling dimension of the two-point correlator associated with $\chi_{\text {loc }}^{\prime \prime}(\omega)$.

Finally, we note that application of a staggered field $h_{s}$ is a relevant perturbation, i.e., it opens a gap at the QCP. The exponent $\eta$ that determines the scaling dimension of $h_{s}$, $\operatorname{dim}\left[h_{s}\right]=D-(1+\eta) / 2=(5-\eta) / 2$, is obtained from the asymptotic behavior of the two-point correlator $\left\langle S_{r}^{+} S_{0}^{-}\right\rangle \propto$ $1 / r^{(1+\eta)}$ for $r \gg a$. We note that the Jordan-Wigner transformation introduces a nonlocal string operator that complicates the computation of this two-point correlator in the fermionic language. However, it is still possible to use the powerful bosonization technique for computing this correlator and obtain $\eta=-1 / 2$ (Giamarchi, 2004).

The relatively straightforward scenario presented for the $d=1$ case is obtained only after making a Jordan-Wigner transformation. A derivation of the same results becomes much more complicated if we insist on using a bosonic language for describing the physics near the $d=1$ QCP. On the other hand, the bosonic description is convenient in higher dimensions because the upper critical dimension is $d_{c}=2$. Therefore, to analyze the critical behavior near the BEC QCP for $d \geq 2$ we express the action in terms of the complex field $\varphi(\mathbf{r}, \tau)$,

$$
\begin{aligned}
\mathcal{L}(\varphi) & =\varphi^{*} \partial_{\tau} \varphi+|\nabla \varphi|^{2}-\tilde{\mu}|\varphi|^{2}+u|\varphi|^{4}-h_{s}\left(\varphi+\varphi^{*}\right), \\
\mathcal{S} & =\int d r^{d} d \tau \mathcal{L}(\varphi(\mathbf{r}, \tau))
\end{aligned}
$$

In this case we included explicitly the term $h_{s}\left(\varphi+\varphi^{*}\right)$ that is a linear coupling between a transverse field $h_{s}$ and the order parameter. For instance, if this is an effective theory for a fieldinduced $X Y$ antiferromagnet, this term represents the linear coupling between an applied staggered field along the $x$ spin direction and the $x$ component of the staggered magnetization. We note that the uniform applied field $H$ enters linearly in the effective chemical potential $\tilde{\mu}$ [see Eq. (9)]. The $T=0$ fixed point again corresponds to $\mu=u=h_{s}=0$. Similarly to the $d=1$ case, the action at the fixed point is invariant under the scale transformation

$$
x^{\prime}=x e^{-l}, \quad \tau^{\prime}=\tau e^{-z l}, \quad \varphi^{\prime}=\varphi e^{l d / 2} .
$$

The scaling dimension of the rest of the variables is obtained by requesting that $\mathcal{S}$ must remain invariant under the scale transformation when we move away from the QCP,

$$
\mu^{\prime}=\mu e^{2 l}, \quad u^{\prime}=u e^{(2-d) l}, \quad h_{s}^{\prime}=h_{s} e^{(2+d / 2) l} .
$$

Again we get that $\nu=1 / 2$ and the scaling dimension of the parameter $u, \operatorname{dim}[u]=d-2$, confirms that $d=2$ is the upper critical dimension. Therefore, we should expect a mean-field behavior for $d \geq 2$ with logarithmic corrections for $d=2$. To obtain the asymptotic behavior of the critical field at low temperatures (phase boundary) we note that $u^{\prime}$ can be made very small by choosing a large value of $e^{l}$. In this way, we can treat the $u^{\prime}$ term of the renormalized action by using perturbation theory. After applying perturbation theory in $u^{\prime}$, we obtain the result that the free energy has a singularity when $\mu^{\prime}=\mu_{c}^{\prime} \propto u^{\prime} T^{d / 2}$. The $T^{d / 2}$ arises from the mean value of $\left|\phi^{\prime}\right|^{2}$ under a quadratic action. Again, the integration over each momentum component leads to a factor $T^{1 / z}$. In terms of the bare coupling constants this condition becomes

$$
H_{c}(T)-H_{c}(0) \propto T^{d / 2} .
$$

For $d=2$ this boundary corresponds to the BerezinskiiKosterlitz-Thouless transition and Eq. (24) is correct up to a logarithmic correction (Fisher and Hohenberg, 1988). A similar mean-field analysis shows that the specific heat and the uniform magnetization at $H=H_{c}(0)$ are proportional to $T^{d / 2}$. The zero-temperature magnetization increases linearly in $H-H_{c}(0)$ for $d \geq 3$. In $d=2$ the uniform magnetization is proportional to $-\left[H-H_{c}(0)\right] / \ln \left[H-H_{c}(0)\right]$ (Fisher and Hohenberg, 1988). The low temperature dependence of the NMR relaxation rate can still be obtained from scaling arguments for $d=2$ (upper critical dimension) and the result is that $1 / T_{1}$ is temperature independent up to logarithmic corrections. However, scaling arguments are no longer applicable in $d=3$ (above the upper critical dimension) and it is necessary to include the effective boson-boson interaction to obtain that $1 / T_{1} \propto T^{3 / 2}$ (Orignac, Citro, and Giamarchi, 2007).

\section{Dimensional reduction}

Here we discuss a frustration-induced dimensional reduction at a BEC QCP. As already pointed out, the main characteristic of the BEC QCP is that the quantum phase transition is driven by a parameter (magnetic field or chemical potential) that couples to a conserved quantity (the $z$ component of the magnetization or number of particles). This observation implies that the ground-state wave function remains invariant in the paramagnetic region. In particular, 
(a)
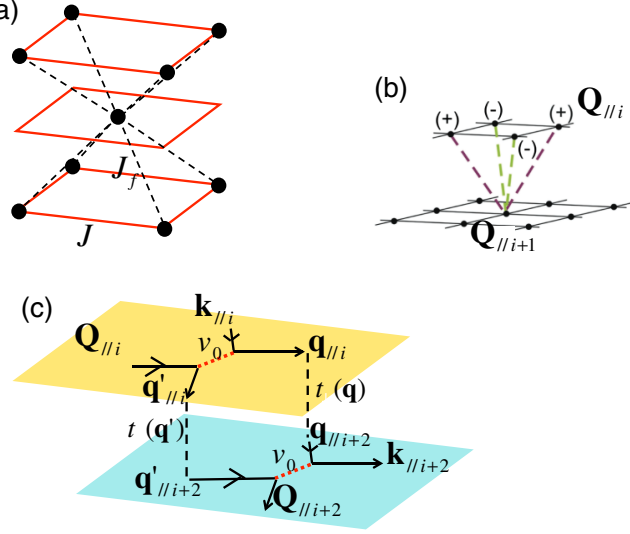

FIG. 2 (color online). (a) Antiferromagnetic $X X Z$ model on a body-centered tetragonal lattice. (b) Blocking of interlayer hopping for bosons in the condensate of each layer. (c) Lowestorder diagram that generates an effective coherent interlayer hopping.

if we are dealing with a two-level system, the ground-state wave function of the paramagnetic region is a product state. For instance, the fully saturated state is a direct product of spin states in each site that are polarized along the field direction. The product state structure of the wave function implies that there are no intersite quantum fluctuations. In this situation, it is possible to obtain the exact single-particle excitation of the paramagnetic phase as we did for the $X X Z$ model (6). In particular, the single-particle dispersion for the saturated phases of an antiferromagnetic $X X Z$ model on a bodycentered tetragonal (bct) is very peculiar. The bct lattice is obtained by stacking square lattices in an $A B A B \ldots$ configuration as is shown in Fig. 2(a).

If the nearest-neighbor intralayer exchange coupling $J$ is dominant and antiferromagnetic, the interlayer exchange $J_{f}$ is completely frustrated. The reason is that $J_{f}$ corresponds to an interaction between a given spin and the sum of the four nearest-neighbor spins in an adjacent layer. The dominant interaction- $J$ forces these four spins to be antiferromagnetically aligned, i.e., their sum is equal to zero and the $J_{f}$ interaction is completely blocked. This is a simple example of geometric frustration. The counterpart of this effect in the particle version of the problem is that the single-boson dispersion $\omega_{\mathbf{q}}=J\left(\cos q_{x}+\cos q_{y}\right)+$ $4 J_{f} \cos q_{z} \cos \left(q_{x} / 2\right) \cos \left(q_{y} / 2\right)$ is minimized for any wave vector $\mathbf{Q}=(\pi, \pi, q)$ that has in-plane components equal to $\mathbf{Q}_{\|}=(\pi, \pi)$ (Jackeli and Zhitomirsky, 2004; Ueda and Totsuka, 2009). In other words, if a boson belongs to a given layer and has momentum $\mathbf{Q}_{\|}=(\pi, \pi)$ (i.e., it is part of the condensate of that layer), it cannot hop to the next layer due to perfect destructive interference between the phases of the four sites that connect with a given site of the adjacent layer [see Fig. 2(b)]. We note that this "perfect blocking" of the interlayer coupling is usually avoided by thermal or quantum fluctuations via the order-from-disorder mechanism (Villain et al., 1980). However, thermal fluctuations do not exist at $T=0$ and intersite quantum fluctuations are also absent in the paramagnetic side of the transition as already anticipated. On the other hand, quantum fluctuations are present on the ordered side and can indeed induce an effective interlayer tunneling for bosons in the condensate of each layer $\left(\left[\mathbf{Q}_{\|}=(\pi, \pi)\right]\right)$ (Maltseva and Coleman, 2005). Figure 2(c) shows the lowest-order diagram that generates this effective hopping. A particle in the condensate of layer $i$ interacts with a second particle in the same layer that has arbitrary momentum $\mathbf{k}_{\| i}$. The outgoing particles have momenta $\mathbf{q}_{\| i}$ and $\mathbf{q}_{\| i}^{\prime}$ $\left(\mathbf{q}_{\| i}+\mathbf{q}_{\| i}^{\prime}=\mathbf{k}_{\| i}+\mathbf{Q}_{\| i}+\mathbf{G}_{\|}\right.$with $\mathbf{G}_{\|}$being a reciprocal lattice vector). Since the bare interlayer hopping amplitudes $t_{\perp}\left(\mathbf{q}_{\| i}\right)$ and $t_{\perp}\left(\mathbf{q}_{\| i}^{\prime}\right)$ are finite for $\mathbf{q}_{\| i}$, and $\mathbf{q}_{\| i}^{\prime}$ different from $\mathbf{Q}_{\| i}$, the two particles can hop to the next-nearest layer and recombine via a second interaction vertex to have final momenta $\mathbf{Q}_{\| i+2}$ and $\mathbf{k}_{\| i+2}$. If we contract the outgoing line with momentum $\mathbf{k}_{\| i+2}$ with the incoming line with momentum $\mathbf{k}_{\| i}$, we end up with an effective (coherent) hopping process that moves particles from the condensate of layer $i$ to the condensate of layer $i+2$. We note that coherent hopping processes between layers with opposite parity (like $i$ and $i+1$ ) are not allowed by the point group symmetry of rotation along the $c$ axis by $\pi / 2$.

It is important to note that the effective hopping amplitude induced by the diagram shown in Fig. 2(c) is proportional to the density of bosons $\rho$ (Batista et al., 2007; Schmalian and Batista, 2008). This is so because the boson in the condensate of layer $i$ has to be assisted by a second boson in order to hop to the condensate of layer $i+2$. Since the bosonic density goes to zero at the BEC QCP, it is natural to ask what is the effective spatial dimensionality of the corresponding fixed point. It turns out that an interlayer hopping amplitude that is proportional to the boson density is a marginal perturbation relative to the decoupled or $d=2$ fixed point (Batista et al., 2007; Schmalian and Batista, 2008). Therefore, although the original system is three dimensional, the BEC QCP is described by the $d=2$ fixed point due to the combination of perfect interlayer frustration and the lack of intersite quantum fluctuations in the paramagnetic phase. For a while, the belief was that this phenomenon of dimensional reduction near a QCP was observed for the first time in the field-induced transition of the quantum dimer compound $\mathrm{BaCuSi}_{2} \mathrm{O}_{6}$ that is also known as Han purple (Sebastian, Tanedo et al., 2006). We note that this is a rather unusual behavior if we consider that dimensionality typically increases near quantum critical points due to amplification of weak couplings between lower-dimensional subsystems. Other works (Krämer et al., 2007; Rösch and Vojta, 2007; Laflorencie and Mila, 2009) emphasized the importance of the existence of inequivalent layers in $\mathrm{BaCuSi}_{2} \mathrm{O}_{6}$ (the intradimer exchange is different for $A$ and $B$ layers: $J_{A}=4.27 \mathrm{meV}$ and $J_{B}=4.72 \mathrm{meV}$ ) (Rüegg et al., 2007). However, a recent work by Mazurenko et al. (2014) has shown that the effective intralayer interaction is ferromagnetic, implying that the observed $d=2$ exponents at the BEC QCP must be attributed to a combination of non equivalent layers with a very weak interlayer interaction. Therefore, the phenomenon of dimensional reduction by frustration remains to be observed in real materials. Natural candidates are $S=1 / 2$ magnets with a bct structure and antiferromagnetic (frustrated) intralayer exchange.

In any case, the dimensional reduction at the BEC QCP of a highly frustrated quantum magnet is a nice example of the novel physical phenomena that arise even in one of the 
simplest types of QCP when we deviate from standard bosonic gases. Given that the BEC QCPs do not satisfy the Harris criterion $(d \nu<2)$ (Harris, 1974), disorder is another important ingredient for inducing novel quantum critical behavior and the so-called Bose glass phase (Fisher et al., 1989). Quantum magnets are ideal for exploring the effects of disorder and test the predictions of Fisher et al. (1989). Indeed, recent results in Br-doped DTN are showing a quite unexpected universal behavior for the QCP that separates the Bose glass from the BEC state (Yu et al., 2012). This is one of the most promising directions for the future of this field.

\section{Theoretical approaches}

\section{Perturbation theory}

Degenerate perturbation theory is the common tool for deriving low-energy effective Hamiltonians of quantum magnets in the presence of an external magnetic field. A low-energy effective Hamiltonian $\tilde{\mathcal{H}}$ is an operator whose spectrum is very similar to the low-energy spectrum of the original Hamiltonian $\mathcal{H}$. If we are only interested in the phenomena that take place well below a certain temperature $T_{0}$, it is enough to work with an effective Hamiltonian that reproduces the spectrum of $\mathcal{H}$ for energies lower than $k_{B} T_{0}$. The main advantage is that $\tilde{\mathcal{H}}$ operates in the smaller Hilbert space of the reduced number of relevant degrees of freedom.

As mentioned, there are three types of quantum paramagnets that form the majority of magnetic-field-induced BEC QCPs: those with weakly coupled spin dimers, those with strong single-ion anisotropy that quenches the local magnetic moment in the absence of an external field, and Haldane chains. Other examples include larger magnetic clusters like integer-spin trimers or tetramers. In the next section we describe the simplest examples of the dimer and single-ion anisotropy cases. In both cases we can apply the formalism of degenerate perturbation theory by expanding in a small parameter. This parameter corresponds to the ratio between exchange interactions responsible for long-range order and the energy scale of the zero-field gap, which could be an intradimer exchange interaction in the first case, or the single-ion anisotropy in the second case. Although the formalism is the same for both classes of magnets, we consider them separately because the effective Hamiltonians can be quite different.

Dimer systems consist of magnetic lattice structures whose units are pairs of spins that interact via a dominant antiferromagnetic exchange constant $J_{0}$. The single dimer spectrum consists of a singlet ground state that is separated from the triplet excited state by an energy difference of order $J_{0}$ (precisely equal to $J_{0}$ for $S=1 / 2$ dimers). The application of an external field lowers the energy of the $S^{z}=1$ triplet state. For high enough field, this triplet state becomes degenerate with the singlet ground state while the energy gap to the other two triplet states remains of order $J_{0}$. For $S=1$ dimers, we also need to consider the $S^{z}=2$ quintet whose energy rapidly decreases as a function of field and becomes degenerate with the single-dimer ground state at high enough fields. If we want to keep only contributions that are of first order in the

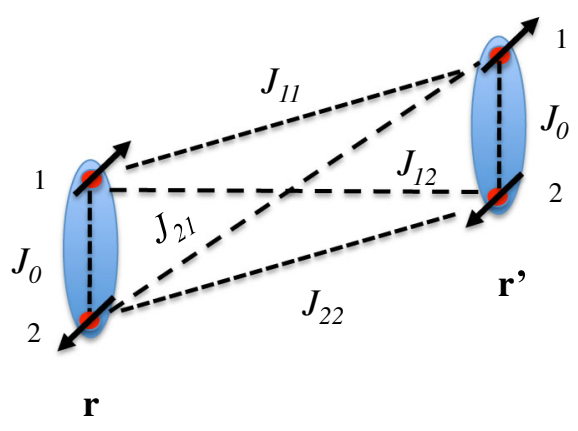

FIG. 3 (color online). Pair of weakly coupled dimers.

interdimer exchange constants $J_{l l^{\prime}}^{\mathbf{r}, \mathbf{r}^{\prime}}$, we need to project our original Hamiltonian into the low-energy subspace generated by the low-energy states (singlet and $S^{z}=1$ triplet for $S=1 / 2$ dimers, singlet, $S^{z}=1$ triplet, and $S^{z}=2$ quintet for $S=1$ dimers, and so on). If $\mathcal{P}$ is the projector into this subspace, we have that $\tilde{\mathcal{H}}=\mathcal{P} \mathcal{H} \mathcal{P}$. The effective twolevel local degree of freedom for $S=1 / 2$ dimers can be represented with a pseudospin $s=1 / 2$ variable or by the equivalent representation in terms of hard-core bosons. (We use lower-case letters for these pseudospin variables in order to distinguish them from the original spin variables, which are denoted by capital letters.) In other words, $\tilde{\mathcal{H}}$ describes a system of interacting $s=1 / 2$ effective moments or, equivalently, a gas of hard-core bosons.

The type of effective interactions that result from weakly coupled dimers can be obtained by considering a pair of dimers with coordinates $\mathbf{r}$ and $\mathbf{r}^{\prime}$ coupled by the most general set of isotropic interdimer exchange constants $J_{l l^{\prime}}$ (see Fig. 3). The corresponding exchange Hamiltonian is

$$
\begin{aligned}
\mathcal{H}_{d}^{r, r^{\prime}}= & J_{0}\left(\mathbf{S}_{\mathbf{r} 1} \cdot \mathbf{S}_{\mathbf{r} 2}+\mathbf{S}_{\mathbf{r}^{\prime} 1} \cdot \mathbf{S}_{\mathbf{r}^{\prime} 2}\right)+\sum_{l, l^{\prime}=1,2} J_{l l^{\prime}}^{\mathbf{r}, \mathbf{r}^{\prime}} \mathbf{S}_{\mathbf{r} l} \cdot \mathbf{S}_{\mathbf{r}^{\prime} l^{\prime}} \\
& -g_{z z} \mu_{B} H \sum_{\mathbf{r} l} S_{\mathbf{r} l}^{z},
\end{aligned}
$$

where $J_{0}$ is the intradimer exchange interaction, and the indices $l, l^{\prime}=\{1,2\}$ specify the particular spin in the dimer. As long as $J_{0} \gg\left|J_{l l^{\prime}}^{\mathbf{r}, \mathbf{r}^{\prime}}\right|$, we can use perturbation theory to include the effect of the interdimer exchange. The effective Hamiltonian to first order in $J_{l l^{\prime}}$ is

$$
\tilde{\mathcal{H}}_{d}^{\mathbf{r}, \mathbf{r}^{\prime}}=\mathcal{P} \mathcal{H}_{d}^{\mathbf{r}, \mathbf{r}^{\prime}} \mathcal{P}
$$

Since the perturbation corresponds to an interaction between spins on different dimers, the following relation holds:

$$
\mathcal{P} \mathbf{S}_{\mathbf{r} l} \cdot \mathbf{S}_{\mathbf{r}^{\prime} l^{\prime}} \mathcal{P}=\mathcal{P} \mathbf{S}_{\mathbf{r} l} \mathcal{P} \cdot \mathcal{P} \mathbf{S}_{\mathbf{r}^{\prime} l^{\prime}} \mathcal{P}
$$

Consequently, to obtain $\tilde{\mathcal{H}}_{d}$ we just need to replace the spin operators of Eq. (25) by the projected spin operators

$$
\tilde{\mathbf{S}}_{\mathbf{r} l}=\mathcal{P} \mathbf{S}_{\mathbf{r} l} \mathcal{P}
$$

where $\nu=x, y, z$. In particular, the projected spin operators for the system under consideration are 


$$
\tilde{S}_{\mathbf{r} l}^{z}=\frac{n_{\mathbf{r}}}{2}, \quad \tilde{S}_{\mathbf{r} l}^{+}=\frac{(-1)^{l}}{\sqrt{2}} b_{\mathbf{r}}^{\dagger}, \quad \tilde{S}_{\mathbf{r} l}^{-}=\frac{(-1)^{l}}{\sqrt{2}} b_{\mathbf{r}} .
$$

Here we have used the hard-core representation for operators acting on the low-energy subspace generated by the singlet and the $S^{z}=1$ triplet of each dimer. The singlet is mapped into the empty state of bosons, while the $S^{z}=1$ triplet corresponds to the state occupied by one boson. The notation for the hard-core boson operators is the same as in Sec. II.A. We note that the internal index $l$ of each dimer has disappeared after the projection because the two spins of the dimer are no longer independent variables after projection into the low-energy sector. This means that each dimer becomes an effective site in the lattice of $\tilde{\mathcal{H}}_{d}$. Therefore, the effective lowenergy model for the two-dimer system of Fig. 3 is a two-site Hamiltonian for a gas of hard-core bosons,

$$
\begin{aligned}
\tilde{\mathcal{H}}_{d}^{r, r^{\prime}}= & t_{r, r^{\prime}}\left(b_{\mathbf{r}}^{\dagger} b_{\mathbf{r}^{\prime}}+b_{\mathbf{r}^{\prime}}^{\dagger} b_{\mathbf{r}}\right)+V_{r, r^{\prime}}\left(n_{\mathbf{r}}-1 / 2\right)\left(n_{\mathbf{r}^{\prime}}-1 / 2\right) \\
& -\mu_{r, r^{\prime}}\left(n_{\mathbf{r}}+n_{\mathbf{r}^{\prime}}\right),
\end{aligned}
$$

where

$$
\begin{aligned}
t_{\mathbf{r}, \mathbf{r}^{\prime}} & =\frac{1}{4}\left(J_{11}^{\mathbf{r}, \mathbf{r}^{\prime}}+J_{22}^{\mathbf{r}, \mathbf{r}^{\prime}}-J_{12}^{\mathbf{r}, \mathbf{r}^{\prime}}-J_{21}^{\mathbf{r}, \mathbf{r}^{\prime}}\right), \\
V_{\mathbf{r}, \mathbf{r}^{\prime}} & =\frac{1}{4}\left(J_{11}^{\mathbf{r}, \mathbf{r}^{\prime}}+J_{22}^{\mathbf{r}, \mathbf{r}^{\prime}}+J_{12}^{\mathbf{r}, \mathbf{r}^{\prime}}+J_{21}^{\mathbf{r}, \mathbf{r}^{\prime}}\right), \\
\mu_{\mathbf{r}, \mathbf{r}^{\prime}} & =-J_{0}+g_{z z} \mu_{B} H-\frac{V_{\mathbf{r}, \mathbf{r}^{\prime}}}{2} .
\end{aligned}
$$

Here $t_{\mathbf{r}, \mathbf{r}^{\prime}}$ is the hopping amplitude between sites $\mathbf{r}$ and $\mathbf{r}^{\prime}, V_{\mathbf{r}, \mathbf{r}^{\prime}}$ is the amplitude of the corresponding off-site density-density interaction, and $\mu_{\mathbf{r}, \mathbf{r}^{\prime}}$ is the chemical potential.

We note that Eq. (30) has been derived for $H>0$. The effective Hamiltonian is identical for negative values of $H$ : $\tilde{\mathcal{H}}_{d}^{\mathbf{r}, \mathbf{r}^{\prime}}(H)=\tilde{\mathcal{H}}_{d}^{\mathbf{r}, \mathbf{r}^{\prime}}(-H)$ due to the time-reversal symmetry of $\mathcal{H}_{d}^{\mathbf{r}, \mathbf{r}^{\prime}}$ (the $S^{z}=1$ triplet must be replaced by the $S^{z}=-1$ triplet). Therefore, without loss of generality, we focus on the $H>0$ case. The extension of this derivation to an infinite and arbitrary lattice of dimers is straightforward. We just need to add the contributions from every pair of dimers that are connected by finite exchange constants $J_{l l^{\prime}}^{\mathrm{r}, \mathbf{r}^{\prime}}$,

$$
\begin{aligned}
\tilde{\mathcal{H}}_{d}= & \sum_{\left\langle\mathbf{r}, \mathbf{r}^{\prime}\right\rangle} t_{\mathbf{r}, \mathbf{r}^{\prime}}\left(b_{\mathbf{r}}^{\dagger} b_{\mathbf{r}^{\prime}}+b_{\mathbf{r}^{\prime}}^{\dagger} b_{\mathbf{r}}\right) \\
& +\sum_{\left\langle\mathbf{r}, \mathbf{r}^{\prime}\right\rangle} V_{\mathbf{r}, \mathbf{r}^{\prime}}\left(n_{\mathbf{r}}-1 / 2\right)\left(n_{\mathbf{r}^{\prime}}-1 / 2\right)-\mu \sum_{\mathbf{r}} n_{\mathbf{r}},
\end{aligned}
$$

with

$$
\mu=-J_{0}+g_{z z} \mu_{B} H-\frac{1}{2} \sum_{\mathbf{r}^{\prime}} V_{\mathbf{r}, \mathbf{r}^{\prime}},
$$

where the angular brackets $\left\langle\mathbf{r}, \mathbf{r}^{\prime}\right\rangle$ indicate that we are summing over the bonds that connect $\mathbf{r}$ and $\mathbf{r}^{\prime} . \tilde{\mathcal{H}}_{d}$ describes a gas of interacting bosons and the model is similar to the one obtained for the $S=1 / 2 \mathrm{XXZ}$ Hamiltonian discussed in Sec. II.A. The main difference is that $\tilde{\mathcal{H}}_{d}$ has two critical fields- $H_{c 1}$ and $H_{c 2}=H_{\text {sat }}$, instead of the single saturation field of $\mathcal{H}_{X X Z}$ (see Fig. 1). This is so because our system of weakly coupled dimers is a gapped quantum paramagnet for $H=0$.

$\tilde{\mathcal{H}}_{d}$ is invariant under the particle-hole transformation $b_{\mathbf{r}}^{\dagger} \rightarrow b_{\mathbf{r}}$ and $\mu \rightarrow-\mu$. This implies that the field-induced ordered phase is symmetric around the $\mu=0$ point that corresponds to $g_{z z} \mu_{B} H_{0}=J_{0}+\frac{1}{2} \sum_{\mathbf{r}^{\prime}} V_{\mathbf{r}, \mathbf{r}^{\prime}}$. $H_{0}$ is at the center of the field-induced dome (phase boundary of the ordered state) on the $H>0$ axis and it corresponds to the field that maximizes the ordering temperature $T_{N}$ (see Fig. 1). The critical field $H_{c 2}$ is mapped into $H_{c 1}$ under the particle-hole transformation, i.e., $H_{c 1}=H_{0}-\left(H_{c 2}-H_{c 1}\right) / 2$ and $H_{c 2}=H_{0}+\left(H_{c 2}-H_{c 1}\right) / 2$. We note that although this transformation is a symmetry of $\tilde{\mathcal{H}}_{d}$, it is not a symmetry of the original Hamiltonian $\mathcal{H}_{d}$. This is an example of an emergent symmetry (Batista and Ortiz, 2004), i.e., a transformation that leaves the low-energy spectrum of $\mathcal{H}_{d}$ invariant up to some order in the perturbative parameter. For the case under consideration, second-order contributions in the interdimer exchange already remove the particle-hole symmetry. Therefore, if the measured boundary of the BEC phase is approximately symmetric around $H_{0}$, we can infer that interdimer exchange is much weaker than $J_{0}$. This is, for instance, the case of $\mathrm{BaCuSi}_{2} \mathrm{O}_{6}$ (Jaime et al., 2004), where the phase diagram of the ordered region in $(T, H)$ is symmetric about $H_{0}$, but it is not the case of $\mathrm{Ba}_{3} \mathrm{Mn}_{2} \mathrm{O}_{8}$ (Samulon et al., 2009).

The most common phases for a gas of interacting bosons, like the one described by $\tilde{\mathcal{H}}_{d}$, are the Mott state, the BEC, and the crystal state (Rice, 2002). As is indicated in Table II, these states correspond to the quantum paramagnet, the $X Y$ antiferromagnet, and Ising-like order, respectively. The Mott state does not break any symmetry, it is gapped, and it has an integer number of bosons per site. For instance, the ground state of $\tilde{\mathcal{H}}_{d}$ is in the quantum paramagnet, or Mott state, for $H<H_{c 1}$ (every dimer is in a singlet state that corresponds to zero bosons per site) and $H>H_{c 2}$ (every dimer is in a $S^{z}=1$ triplet state that corresponds to one hard-core boson per site). The $X Y$ AFM, or BEC state, is stabilized between $H_{c 1}$ and $H_{c 2}$. Here the hopping amplitudes $t_{\mathbf{r}, \mathbf{r}^{\prime}}$ dominate over the offsite repulsions $V_{\mathbf{r}, \mathbf{r}^{\prime}}$. On the other hand, dominant repulsive terms can lead to a Bose crystal rather than a BEC when boson filling factors are commensurate. In this case the spectrum is usually gapped. The experimental signature of the crystal state is the appearance of plateaus in the field dependence of the uniform magnetization. The Shastry-Sutherland (Shastry and Sutherland, 1981) compound $\mathrm{SrCu}_{2}\left(\mathrm{BO}_{3}\right)_{2}$ is a prominent example of field-induced crystallization in a spin-dimer

TABLE II. Corresponding spin and boson variables under the spinparticle transformations discussed in the main text.

\begin{tabular}{lc}
\hline \hline Spin & Particle \\
\hline$\left\langle S_{j}^{z}\right\rangle$ & $\left\langle n_{j}^{b}\right\rangle$ \\
$g_{z z} \mu_{B} H$ & $\mu$ \\
$\left\langle m_{x} \pm i m_{y}\right\rangle$ & $\left\langle b_{j}^{\dagger}\right\rangle,\left\langle b_{j}\right\rangle$ \\
Superfluid density & Spin stiffness \\
Ising-like ordering & Crystalization \\
$X Y$-like ordering & BEC \\
Gapped quantum paramagnet & Mott insulator \\
\hline \hline
\end{tabular}


quantum paramagnet (Smith and Keszler, 1991; Kageyama, Onizuka et al., 1999; Kageyama, Yoshimura 1999; Misguich, Jolicoeur, and Girvin, 2001; Kodama et al., 2002; Shastry and Kumar, 2002; Miyahara and Ueda, 2003; Sebastian et al., 2008).

From Eqs. (31), one can see that the ratio $t_{\mathbf{r}, \mathbf{r}^{\prime}} / V_{\mathbf{r}, \mathbf{r}^{\prime}}$ is controlled by the degree of frustration between the different exchange interactions that connect a given pair of dimers. In particular, we have that $t_{\mathbf{r}, \mathbf{r}^{\prime}}=0$ when the four exchange couplings are the same (fully frustrated case). To understand the origin of this cancellation we need to note that a hopping process corresponds to the exchange of singlet and triplet states between the two dimers. While the triplet state is symmetric under the exchange of the two dimer sites 1 and 2, the singlet state is antisymmetric. The exchange terms $J_{12}^{\mathbf{r}, \mathbf{r}^{\prime}}$ and $J_{21}^{\mathbf{r}, \mathbf{r}^{\prime}}$ can be obtained from the terms $J_{11}^{\mathbf{r}, \mathbf{r}^{\prime}}$ and $J_{22}^{\mathbf{r}, \mathbf{r}^{\prime}}$ by exchanging the two sites of the second dimer. This implies that the $J_{12}^{\mathbf{r}, \mathbf{r}^{\prime}}$ and $J_{21}^{\mathbf{r}, \mathbf{r}^{\prime}}$ contributions to the effective hopping $t_{\mathbf{r}, \mathbf{r}^{\prime}}$ must have opposite signs relative to contributions from $J_{11}^{\mathbf{r}, \mathbf{r}^{\prime}}$ and $J_{22}^{\mathbf{r}, \mathbf{r}^{\prime}}$ [see Eqs. (31)]. On the other hand, the four amplitudes contribute with a positive sign to $V_{\mathbf{r}, \mathbf{r}^{\prime}}$ because this effective repulsion results from the Ising terms $S_{\mathbf{r} l}^{z} S_{\mathbf{r}^{\prime} l^{\prime}}^{z}$, which are all positive if there is a triplet state in each dimer. Therefore, the degree of frustration in the interdimer couplings is the key ingredient that determines whether the field-induced ordered state is a crystal or a BEC. In general, the fieldinduced ordered region can also include BEC and crystal phases in different intervals of magnetic fields. Moreover, these two phases can also coexist in certain windows of magnetic field leading to the so-called spin supersolid state on the lattice (Laflorencie and Mila, 2007; Sengupta and Batista, 2007a, 2007b; Schmidt et al., 2008; Albuquerque et al., 2011; Peters, McCulloch, and Selke, 2012). In other words, interdimer frustration provides a natural mechanism for realizing a gas of bosons with dominant off-site repulsions that are a precondition for stabilizing spin supersolid states. We note that this is specific to dimers, and is not the case for singlespin systems in the nearly isotropic Heisenberg magnets for which the hopping amplitude and off-site repulsion are of the same order (see Sec. II.A).

To complete the previous discussion, it is instructive to apply the Matsubara-Matsuda transformation (Matsubara and Matsuda, 1956) to $\tilde{\mathcal{H}}_{d}$,

$$
\tilde{\mathcal{H}}_{d}=\sum_{\left\langle\mathbf{r}, \mathbf{r}^{\prime}\right\rangle}\left[\frac{t_{\mathbf{r}, \mathbf{r}^{\prime}}}{2}\left(s_{\mathbf{r}}^{x} s_{\mathbf{r}^{\prime}}^{x}+s_{\mathbf{r}}^{y} s_{\mathbf{r}^{\prime}}^{y}\right)+V_{\mathbf{r}, \mathbf{r}^{\prime}} s_{\mathbf{r}^{z}}^{z} s_{\mathbf{r}^{\prime}}^{z}\right]-\mu \sum_{\mathbf{r}} s_{\mathbf{r}}^{z}
$$

The pseudospin-1/2 variable $\mathbf{s}_{\mathbf{r}}$ provides an alternative description of the two-level singlet-triplet system $\left(s_{\mathbf{r}}^{z}=1 / 2\right.$ corresponds to the triplet state while $s_{\mathbf{r}^{\prime}}^{z}=-1 / 2$ corresponds to the singlet state). A small $t_{\mathbf{r}, \mathbf{r}^{\prime}} / V_{\mathbf{r}, \mathbf{r}^{\prime}}$ ratio corresponds to a strong easy-axis exchange anisotropy in this effective $X X Z$ model. Therefore, by starting with an isotropic spin system, we managed to produce an effective low-energy model that can have a very anisotropic exchange interaction. The Zeeman anisotropy produced by the application of a large magnetic field has been converted into a strong effective exchange anisotropy by combining strong dimerization and interdimer frustration. As explained earlier, this property opens the door for realizing novel and exotic field-induced magnetic orderings in quantum dimer systems. There are other exotic states such as Bose metals (spin liquids) that can be stabilized in very frustrated lattices or Bose glasses that can appear in presence of disorder. Although this is a fascinating subject, a detailed discussion of these states of matter is beyond the scope of this review.

The effective Hamiltonians are more constrained in the case of magnets whose local moments are quenched by a strong single-ion anisotropy. The spins of these magnets have to be $S=1$ or higher because of the requirement of single-ion anisotropy. The simplest and prototypical example corresponds to $S=1$ and a large uniaxial anisotropy term $D\left(S^{z}\right)^{2}$, with $D \gg\left|J_{\mathbf{r}, \mathbf{r}^{\prime}}\right|\left(J_{\mathbf{r}, \mathbf{r}^{\prime}}\right.$ are the exchange constants between spins located at sites $\mathbf{r}$ and $\mathbf{r}^{\prime}$ of the magnetic lattice). The compound $\mathrm{NiCl}_{2}-4 \mathrm{SC}\left(\mathrm{NH}_{2}\right)_{2}$ (Paduan-Filho et al., 1981, 2009;Paduan-Filho, Gratens, and Oliveira, 2004b; Zapf et al., 2006; Zvyagin et al., 2007; Chiatti et al., 2008, 2009; Cox et al., 2008; Reyes, Paduan-Filho, and Continentino, 2008; Yin et al., 2008; Zvyagin et al. 2008; Sun et al., 2009; Zherlitsyn et al., 2009; Kohama et al., 2011; Zapf et al. 2011; Paduan-Filho, 2012; Psaroudaki et al., 2012), also known as DTN, provides a natural realization of this situation. If the dominant terms of the magnetic system are U(1) invariant, the spin Hamiltonian for a pair of neighboring ions has the general form

$$
\begin{aligned}
\mathcal{H}_{s}^{\mathbf{r}, \mathbf{r}^{\prime}}= & D\left[\left(S_{\mathbf{r}}^{z}\right)^{2}+\left(S_{r^{\prime}}^{z}\right)^{2}\right]-g_{z z} \mu_{B} H\left(S_{\mathbf{r}}^{z}+S_{\mathbf{r}^{\prime}}^{z}\right) \\
& +J_{\mathbf{r}, \mathbf{r}^{\prime}}\left(\gamma S_{\mathbf{r}^{\prime}}^{z} S_{\mathbf{r}^{\prime}}^{z}+S_{\mathbf{r}}^{x} S_{\mathbf{r}^{\prime}}^{x}+S_{\mathbf{r}}^{y} S_{\mathbf{r}^{\prime}}^{y}\right) .
\end{aligned}
$$

In this case, the low-energy subspace corresponds to the direct product of the single-ion subspaces generated by the $S^{z}=0$ and $S^{z}=1$ states (we keep assuming that the magnetic field is positive). By repeating the projection process similar to the one described by Eqs. (26), (27), (28), and using the new projected spin operators,

$$
\tilde{S}_{\mathbf{r}}^{z}=n_{\mathbf{r}}, \tilde{S}_{\mathbf{r}}^{+}=\sqrt{2} b_{\mathbf{r}}^{\dagger}, \tilde{S}_{\mathbf{r}}^{-}=\sqrt{2} b_{\mathbf{r}},
$$

( $n_{\mathbf{r}}=1$ corresponds to the $S^{z}=1$ state while $n_{\mathbf{r}}=0$ corresponds to the $S^{z}=0$ state) we obtain the effective low-energy Hamiltonian for $\mathcal{H}_{s}^{\mathbf{r}, \mathbf{r}^{\prime}}$,

$$
\begin{aligned}
\tilde{\mathcal{H}}_{s}^{\mathbf{r}, \mathbf{r}^{\prime}}= & t_{\mathbf{r}, r^{\prime}}\left(b_{\mathbf{r}}^{\dagger} b_{\mathbf{r}^{\prime}}+b_{\mathbf{r}^{\prime}}^{\dagger} b_{\mathbf{r}}\right)+V_{\mathbf{r}, \mathbf{r}^{\prime}}\left(n_{\mathbf{r}}-1 / 2\right)\left(n_{\mathbf{r}^{\prime}}-1 / 2\right) \\
& -\mu_{\mathbf{r}, \mathbf{r}^{\prime}}\left(n_{\mathbf{r}}+n_{\mathbf{r}^{\prime}}\right),
\end{aligned}
$$

where

$$
t_{\mathbf{r}, \mathbf{r}^{\prime}}=J_{\mathbf{r}, \mathbf{r}^{\prime}}, V_{\mathbf{r}, \mathbf{r}^{\prime}}=\gamma J_{\mathbf{r}, \mathbf{r}^{\prime}}, \mu_{\mathbf{r}, \mathbf{r}^{\prime}}=-D+g_{z z} \mu_{B} H-\frac{V_{\mathbf{r}, \mathbf{r}^{\prime}}}{2} .
$$

Although the effective Hamiltonian (37) has the same form as $\tilde{\mathcal{H}}_{d}$, it is clear from Eq. (38) that the Hamiltonian parameters are more restricted than in the case of dimerized spin systems [see Eqs. (31)]. The effective exchange anisotropy for the dimerized compounds can be easy-plane or easy-axis depending on the relative strength of the four interdimer exchange interactions $J_{l l^{\prime}}^{\mathbf{r}, \mathbf{r}^{\prime}}$. In contrast, since $\gamma \simeq 1$, the effective exchange anisotropy of $\tilde{\mathcal{H}}_{s}^{\mathbf{r}, \mathbf{r}^{\prime}}$ is easy-plane or $X Y$-like because 
the hopping amplitude is similar to the nearest-neighbor repulsion. We note that the exchange anisotropy of the original Hamiltonian (35) is usually small $(\gamma \simeq 1)$ for magnetic ions with relatively small spin-orbit coupling. Therefore, we expect that the field-induced BEC phase should be the most common type of ordering for this class of axially symmetric quantum paramagnets. That is indeed the case for DTN.

\section{Generalized spin-wave theory}

Once we have an adequate original or effective spin Hamiltonian $\mathcal{H}$ for describing the quantum magnet under consideration, we need to find an approach for describing the low-temperature magnetic ordering and low-energy excitations. The simplest and most popular approach is to propose a mean-field (MF) variational ground state of the form

$$
\left|\psi_{\mathrm{MF}}\left(\left\{\kappa_{\mathbf{r}}^{j}\right\}\right)\right\rangle=\otimes_{\mathbf{r}}\left|\psi_{\mathbf{r}}\left(\left\{\kappa_{\mathbf{r}}^{j}\right\}\right)\right\rangle
$$

where $\mathbf{r}$ is the coordinate of each unit cell and $\left|\psi_{\mathbf{r}}\left(\left\{\kappa_{\mathbf{r}}^{j}\right\}\right)\right\rangle$ is a local wave function for the spin degrees of freedom in the unit cell at $\mathbf{r}$. The variational parameters $\left\{\kappa_{\mathbf{r}}^{j}\right\}$ are determined by minimizing the mean energy $\left\langle\psi_{\mathrm{MF}}\left(\left\{\kappa_{\mathbf{r}}^{j}\right\}\right)|\mathcal{H}| \psi_{\mathrm{MF}}\left(\left\{\kappa_{\mathbf{r}}^{j}\right\}\right)\right\rangle$. It is clear that these parameters are a function of the applied magnetic field and the nature of $\left|\psi_{\mathrm{MF}}\left(\left\{\kappa_{\mathbf{r}}^{j}\right\}\right)\right\rangle$ must change qualitatively at the critical field $H_{c 1}$. In particular, if the transition corresponds to a BEC QCP, we have seen that this mean-field description will provide the correct exponents for $d \geq 2$. Low-energy excitations for this mean-field ground state are obtained by including Gaussian or quadratic fluctuations as is usually done in the traditional spin-wave treatment. However, the method that we describe here (Muniz, Kato, and Batista, 2013) differs from the traditional spin-wave approach. The main difference arises from the variational space of the mean-field wave function. In the traditional spin-wave theory, the most general mean-field wave function contains two variational parameters $\theta_{\mathbf{r}}$ and $\phi_{\mathbf{r}}$ per spin ( $\mathbf{r}$ is the coordinate of each spin). There is a one-to-one correspondence between these mean-field states and a classical spin configuration in which the orientation of the classical spin at site $\mathbf{r}$ is determined by the spherical angles $\theta_{\mathbf{r}}$ and $\phi_{\mathbf{r}}$. This parametrization is usually enough for mean-field descriptions of Heisenberg-like spin models with one spin per unit cell and weak single-ion anisotropy. However, a more complete parametrization is required for more general quantum magnets.

For instance, the mean-field description of the ground state of DTN in the absence of magnetic field is a direct product of the $S_{\mathbf{r}}^{z}=0$ state $\left|S_{\mathbf{r}}^{z}=0\right\rangle$ on every ion. This mean-field state has no classical counterpart because $\left\langle S_{\mathbf{r}}^{z}=0\left|S_{\mathbf{r}}^{\nu}\right| S_{\mathbf{r}}^{z}=0\right\rangle=0$ for $\nu=\{x, y, z\}$. This observation leads to a very natural question: What is the most general parametrization of $\left|\psi_{\mathrm{MF}}\left(\left\{\kappa_{\mathbf{r}}^{j}\right\}\right)\right\rangle$ ? The answer depends on the dimension $N$ of the local Hilbert space of each unit cell. The parametrization of an arbitrary state $\left|\psi_{\mathbf{r}}\left(\left\{\kappa_{\mathbf{r}}^{j}\right\}\right)\right\rangle$ for the unit cell $\mathbf{r}$ requires a total of $N-1$ complex variational parameters (note that the wave function is defined up to a phase). A pair of arbitrary states is connected by a unitary transformation that belongs to the $\mathrm{SU}(N)$ group, in contrast to the reduced $\mathrm{SU}(2)$ group that connects all possible variational states of the traditional spin-wave approach. If we are dealing with a system like DTN with one spin $S=1$ per unit cell, we need four variational parameters per unit cell (two complex numbers) (Muniz, Kato, and Batista, 2013). The physical origin of this freedom is that a local Hilbert space of dimension $N$ admits local order parameters that are more general than the magnetization. The local order parameter of our $S=1$ example has eight independent components: besides the three components of the local magnetization vector $\left(S_{\mathbf{r}}^{x}, S_{\mathbf{r}}^{y}, S_{\mathbf{r}}^{z}\right)$, there are the five nematic components of the symmetric and traceless tensor $3\left(S^{z}\right)^{2}-2, \quad\left(S^{x}\right)^{2}-\left(S^{y}\right)^{2}, S^{x} S^{y}+S^{y} S^{x}, S^{x} S^{z}+S^{y} S^{z}$, and $S^{z} S^{y}+S^{y} S^{z}$. In general, the $N^{2}-1$ components of the local order parameter are generators of the $\mathrm{SU}(N)$ group. Strong inhomogeneities in the exchange constants or strong easy axis anisotropies can stabilize states with nonzero mean values of components of the local order parameter that are orthogonal to the magnetization. For instance, the $\left|S_{\mathbf{r}}^{z}=0\right\rangle$ state that is relevant for DTN has a net nematic component but no net magnetization.

Since the components of the most general local order parameter are generators of the $\mathrm{SU}(N)$ group, it is clear that these generators provide a more appropriate language for including the Gaussian fluctuations. We note that these quantum fluctuations, induced by the exchange interaction between different magnetic moments, lead to small deviations of the local order parameter around its mean value. Schwinger bosons (SBs) provide a useful representation of the generators of the $\mathrm{SU}(N)$ group in the fundamental representation (Auerbach, 1998),

$$
S_{\mathbf{r}}^{m m^{\prime}}=b_{\mathbf{r} m}^{\dagger} b_{\mathbf{r} m^{\prime}},
$$

where the indices $1 \leq m, m^{\prime} \leq N$ run over the $N$ states that form the basis of the local Hilbert space and the SBs satisfy the constraint

$$
\sum_{m} b_{\mathbf{r} m}^{\dagger} b_{\mathbf{r} m}=N \mathcal{S}
$$

$\mathcal{S}=1 / N$ for the case of interest because we are working with the fundamental representation of $\mathrm{SU}(N)$. However, under special circumstances it could be more convenient to work with a higher representation of $\mathrm{SU}(N)$. The operators $S^{m m^{\prime}}$ satisfy the $\operatorname{su}(N)$ algebra,

$$
\left[S_{\mathbf{r}}^{m m^{\prime}}, S_{\mathbf{r}^{\prime}}^{l l^{\prime}}\right]=\left(\delta_{m^{\prime} l} S_{\mathbf{r}}^{m l^{\prime}}-\delta_{m l^{\prime}} S_{\mathbf{r}}^{l m^{\prime}}\right) \delta_{\mathbf{r r}^{\prime}}
$$

One useful property of the fundamental representation is that any local operator can be expressed as a linear combination of the identity and the generators $S^{\mathrm{mm}}$. In particular, we are interested in expressing the spin operators as a linear combination of the generators $S^{m m^{\prime}}$,

$$
S_{l \mathbf{r}}^{\nu}=\sum_{m m^{\prime}} b_{m \mathbf{r}}^{\dagger} \mathcal{S}_{l m m^{\prime}}^{\nu} b_{m^{\prime} \mathbf{r}}
$$

where $l$ is an internal index of the unit cell and $\nu=\{x, y, z\}$. $\mathcal{S}_{l}^{\nu}$ is then the matrix associated to the spin operator $S_{l \mathbf{r}}^{\nu}$ in the $\mathrm{SB}$ representation of generators of $\mathrm{SU}(N)$. Similarly,

$$
\left(S_{l \mathbf{r}}^{\nu}\right)^{n}=\sum_{m m^{\prime}} b_{m \mathbf{r}}^{\dagger} \mathcal{O}_{l m m^{\prime}}^{\nu, n} b_{m^{\prime} \mathbf{r}}
$$


where $\mathcal{O}^{\nu, n}$ is the matrix associated with the $\left(S_{l \mathbf{r}^{\prime}}^{\nu}\right)^{n}$ and $\mathcal{O}_{l}^{\nu, 1}=\mathcal{S}_{l}^{\nu}$.

The Hamiltonian $\mathcal{H}$ under consideration is always a polynomial function of the spin operators $S_{l \mathbf{r}^{\prime}}^{\nu}$. Therefore, the SB representation of $\mathcal{H}$ is obtained by replacing the operators $\left(S_{l \mathbf{r}^{\prime}}^{\nu}\right)^{n}$ with the bilinear form (43)

$$
\mathcal{H}\left(\left\{S_{l \mathbf{r}}^{\nu}\right\}\right) \rightarrow \mathcal{H}\left(\left\{\sum_{m m^{\prime}} b_{m \mathbf{r}}^{\dagger} \mathcal{S}_{l m m^{\prime}}^{\nu} b_{m^{\prime} \mathbf{r}}\right\}\right) .
$$

Equation (44) implies that Hamiltonian terms that involve spins from the same unit cell are bilinear forms in the SBs. However, exchange terms between spins on different sites are biquadratic because each site brings a bilinear form.

It is also important to note that any local state can be expressed as a linear combination of the states $b_{\mathbf{r} m}^{\dagger}|\emptyset\rangle$. This is true in particular for the local states $\left|\psi_{\mathbf{r}}\left(\left\{\kappa_{\mathbf{r}}^{j}\right\}\right)\right\rangle$ that appear in the lowest energy mean-field or product state (39),

$$
\left|\psi_{\mathbf{r}}\left(\left\{\kappa_{\mathbf{r}}^{j}\right\}\right)\right\rangle=\sum_{j=1, N} \kappa_{\mathbf{r}}^{j} b_{j}^{\dagger}|\emptyset\rangle .
$$

The rest of the procedure is formally similar to the traditional spin-wave approach. The first step is to apply a unitary transformation [rotation in $\mathrm{SU}(N)$ ] that transforms $b_{1 \mathbf{r}}^{\dagger}$ into $\tilde{b}_{1 \mathbf{r}}^{\dagger}=\sum_{j=1, N} \kappa_{\mathbf{r}}^{j} b_{j \mathbf{r}}^{\dagger}$,

$$
\tilde{b}_{m \mathbf{r}}^{\dagger}=\sum_{l} \mathcal{U}_{m l}^{\mathbf{r}} b_{l \mathbf{r}}^{\dagger}
$$

It is clear that the unitary transformation $\mathcal{U}^{\mathbf{r}}$ is not unique. The mean-field state (39) can be regarded as a condensation of the new $\tilde{b}_{1 \mathbf{r}}^{\dagger}$ bosons,

$$
\left|\psi_{\mathrm{MF}}\left(\left\{\kappa_{\mathbf{r}}^{j}\right\}\right)\right\rangle=\otimes_{\mathbf{r}} \tilde{b}_{\mathbf{r}}^{\dagger}|\emptyset\rangle .
$$

In other words, the mean-field approximation of the ground state is the state with $\tilde{n}_{1 \mathbf{r}}=\tilde{b}_{1 \mathbf{r}}^{\dagger} \tilde{b}_{1 \mathbf{r}}=1$ and $\tilde{n}_{m \mathbf{r}}=0$ $(1<m \leq N)$. If we assume that fluctuations will induce small changes in these occupation numbers, we can introduce the following generalization of the Holtstein-Primakoff transformation:

$$
\tilde{b}_{1 \mathbf{r}}^{\dagger} \simeq \sqrt{\mathcal{S} N-\sum_{j=2, N} \tilde{b}_{j \mathbf{r}}^{\dagger} \tilde{b}_{j \mathbf{r}}}
$$

which follows from the constraint (41). The rest of the procedure is very straightforward. In the first place we need to express $\mathcal{H}$ as a function of the new SB operators,

$$
\mathcal{H}\left(\left\{\sum_{m m^{\prime}} b_{m \mathbf{r}}^{\dagger} \mathcal{O}_{l m m^{\prime}}^{\nu, n} b_{m^{\prime} \mathbf{r}}\right\}\right) \rightarrow \mathcal{H}\left(\left\{\sum_{m m^{\prime}} \tilde{b}_{m \mathbf{r}}^{\dagger} \tilde{\mathcal{O}}_{l m m^{\prime}}^{\nu, n} \tilde{b}_{m^{\prime} \mathbf{r}}\right\}\right),
$$

with $\tilde{\mathcal{O}}_{l}^{\nu, n}=\mathcal{U}^{\mathbf{r} \dagger} \mathcal{O}_{l}^{\nu, n} \mathcal{U}^{\mathbf{r}}$. In the second place, we need to keep the Hamiltonian terms up to quadratic order in the SB operators $\tilde{b}_{m \mathbf{r}}^{\dagger}$ and $\tilde{b}_{m^{\prime} \mathbf{r}}\left(1<m, m^{\prime} \leq N\right)$,

$$
\mathcal{H} \simeq \mathcal{H}^{(0)}+\mathcal{H}^{(1)}+\mathcal{H}^{(2)}+\cdots,
$$

where $\mathcal{H}^{(0)}=\left\langle\psi_{\mathrm{MF}}\left(\left\{\kappa_{\mathbf{r}}^{j}\right\}\right)|\mathcal{H}| \psi_{\mathrm{MF}}\left(\left\{\kappa_{\mathbf{r}}^{j}\right\}\right)\right\rangle$ is the mean-field energy. The linear contributions cancel, e.g., $\mathcal{H}^{(1)}=0$, because we minimized the mean-field energy as a function of the variational parameters $\kappa_{\mathbf{r}}^{j}$. Finally, $\mathcal{H}^{(2)}$ is the quadratic contribution that can be diagonalized by going to momentum space and applying a standard Bogoliubov transformation. This approximation can be regarded as a $1 / N \mathcal{S}$ expansion, which is a generalization of the traditional $1 / S$ expansion.

It is important to stress that the local order parameter space of the traditional spin-wave approach and the $\mathrm{SU}(N)$ case for $\mathcal{S}=1 / N$, that we just introduced, are completely different. In the former case, the local order parameter is the local magnetization and small oscillations around its equilibrium value can be described by a single Holstein-Primakoff boson (Holstein and Primakoff, 1940). In the second case, the local order parameter space is larger and consequently we need to introduce a larger number, $N-1$, of Holstein-Primakoff bosons in order to describe the small oscillations around its equilibrium value. Therefore, the two approximations are different even when the local order parameter is the local magnetization (Muniz, Kato, and Batista, 2013).

Our generalized spin-wave approach is indeed useful for describing quantum paramagnets in a magnetic field because the local order parameter evolves from a nonmagnetic situation, in absence of an external field, to a magnetically ordered state for $H>H_{c 1}$. For instance, in the case of weakly coupled dimers, the unit cell has two spins and the local Hilbert space consists of one singlet and three triplets. This implies that $N=4$, i.e., we need to introduce four SB bosons, one for creating the singlet state and three for creating the triplets. In the absence of a magnetic field, the optimal meanfield wave function (39) is a direct product of singlets in each dimer and consequently we need to condense the boson that creates the singlet state. This so-called bond operator representation (Chubukov, 1989; Sachdev and Bhatt, 1990) is then a particular case of the generalized spin-wave approach. A nice application of this formalism to the case of $\mathrm{TlCuCl}_{3}$ can be found in Matsumoto et al. (2002, 2004).

Another example of application of the generalized spinwave approach to DTN ( $S=1$ magnetic moment quenched by strong single-ion anisotropy) is given by Zapf et al. (2006); Kohama et al. (2011); and Zhang et al. (2013). The relevant $S=1$ spin Hamiltonian is a particular case of Eq. (35),

$$
\mathcal{H}=\sum_{\mathbf{r}, \nu} J_{\nu} \mathbf{S}_{\mathbf{r}} \cdot \mathbf{S}_{\mathbf{r}+\mathbf{e}_{\nu}}+D \sum_{\mathbf{r}}\left(S_{\mathbf{r}}^{z}\right)^{2}-h \sum_{\mathbf{r}} S_{\mathbf{r}}^{z},
$$

where $\mathbf{e}_{\nu}$ are the primitive vectors of the lattice, $\nu=\{a, b, c\}$, and $h=g_{z z} \mu_{B} H$. The dominant single-ion uniaxial anisotropy $D=8.9 \mathrm{~K}$ splits the Ni $S=1$ triplet into an $S^{z}=0$ ground state and an $S^{z}= \pm 1$ excited doublet. The antiferromagnetic exchange coupling between $\mathrm{Ni}$ ions is $J_{c}=2.2 \mathrm{~K}$ along the $c$ axis and $J_{a}=0.18 \mathrm{~K}$ along the $a$ and $b$ axes, while the gyromagnetic factor along the $c$ axis is $g_{z z}=2.26$ (Zvyagin et al., 2007). DTN is a quasi-one-dimensional magnet because $J_{c} / J_{a b} \gtrsim 10$.

The dimension of the local Hilbert space is $N=3\left(S^{z}=1\right.$, $0,-1)$. Therefore, it is convenient to use $\mathrm{SU}(3) \mathrm{SBs} b_{1 \mathbf{r}}^{\dagger}, b_{0 \mathbf{r}}^{\dagger}$, $b_{1 \mathbf{r}}^{\dagger}$ that create eigenstates of $S_{\mathbf{r}}^{z}$ with eigenvalues $1,0,-1$, respectively. The SBs obey the constraint (41) 


$$
\sum_{m=-1,0,1} b_{\mathbf{r} m}^{\dagger} b_{\mathbf{r} m}=1
$$

The spin operators in this representation are

$$
S_{\mathbf{r}}^{z}=n_{\mathbf{r} \uparrow}-n_{\mathbf{r} \downarrow}, S_{\mathbf{r}}^{+}=\left(S_{\mathbf{r}}^{-}\right)^{\dagger}=\sqrt{2}\left(b_{\mathbf{r} \uparrow}^{\dagger} b_{\mathbf{r} 0}+b_{\mathbf{r} 0}^{\dagger} b_{\mathbf{r} \downarrow}\right),
$$

where the indices $\{\uparrow, 0, \downarrow\}$ correspond to $m=\{1,0,-1\}$ and $n_{\mathbf{r} m}=b_{\mathbf{r} m}^{\dagger} b_{\mathbf{r} m}$. Instead of using the Holstein-Primakoff approximation (49) (Holstein and Primakoff, 1940), we enforce the constraint at a mean-field level by introducing spatially uniform Lagrange multiplier $\mu$ :

$$
\hat{\mathcal{H}}=\mathcal{H}+\mu \sum_{\mathbf{r}}\left(b_{\mathbf{r} \uparrow}^{\dagger} b_{\mathbf{r} \uparrow}^{\dagger}+b_{\mathbf{r} \downarrow}^{\dagger} b_{\mathbf{r} \downarrow}^{\dagger}+b_{\mathbf{r} 0}^{\dagger} b_{\mathbf{r} 0}^{\dagger}-1\right) .
$$

As discussed next, this procedure has one advantage and one disadvantage relative to the more traditional HolsteinPrimakoff approach (Holstein and Primakoff, 1940). The lowfield quantum paramagnet is described by the mean-field state,

$$
\left|\psi_{\mathrm{MF}}\right\rangle=\otimes_{\mathbf{r}} b_{0 \mathbf{r}}^{\dagger}|\emptyset\rangle .
$$

By using the spin representation (54) with the mean-field value for $\left\langle b_{0 \mathbf{r}}^{(\dagger)}\right\rangle=s$ and neglecting terms of order higher than quadratic in the other two bosonic operators $b_{\uparrow(\downarrow) \mathbf{r}}^{(\dagger)}$, we obtain the generalized spin-wave Hamiltonian in the harmonic approximation

$$
\hat{\mathcal{H}}=E_{0}+\sum_{\mathbf{k}, \sigma}\left[A_{\mathbf{k} \sigma} \hat{b}_{\mathbf{k} \sigma}^{\dagger} \hat{b}_{\mathbf{k} \sigma}+\frac{B_{\mathbf{k}}}{2}\left(\hat{b}_{\mathbf{k} \sigma}^{\dagger} \hat{b}_{-\mathbf{k} \bar{\sigma}}^{\dagger}+\text { H.c. }\right)\right],
$$

with $A_{\mathbf{k} \sigma}=\left(\mu+s^{2} \epsilon_{\mathbf{k}}-h_{\sigma}\right)$ and $B_{\mathbf{k}}=s^{2} \epsilon_{\mathbf{k}}$, where $E_{0}=$ $N(\mu-D)\left(s^{2}-1\right)$ is the bare ground state energy, $N$ is the number of sites, $\sigma=\{\uparrow, \downarrow\}, h_{\sigma}= \pm h, \bar{\sigma}=-\sigma, \hat{b}_{\mathbf{k} \sigma}^{(\dagger)}$ are the Fourier-transformed bosonic operators, and $\epsilon_{\mathbf{k}}=2 \sum_{\nu} J_{\nu} \cos k_{\nu}$. The anomalous terms indicate that bosons with opposite $S^{z}$ are created and annihilated in the ground state. These are the quantum fluctuations that lead to renormalization of the quasiparticle dispersion relation. The Hamiltonian (57) is diagonalized by the Bogoliubov transformation

$$
\hat{b}_{\mathbf{k} \sigma}=u_{\mathbf{k}} \beta_{\mathbf{k} \sigma}+v_{\mathbf{k}} \beta_{-\mathbf{k} \bar{\sigma}}^{\dagger},
$$

where $u_{\mathbf{k}} v_{\mathbf{k}}=B_{\mathbf{k}} / 2 \omega_{\mathbf{k}}^{0}, \quad u_{\mathbf{k}}^{2}+v_{\mathbf{k}}^{2}=\left(\mu+s^{2} \epsilon_{\mathbf{k}}\right) / \omega_{\mathbf{k}}^{0}$, and $\omega_{\mathbf{k}}^{0}=\sqrt{\mu^{2}+2 \mu s^{2} \epsilon_{\mathbf{k}}}$. The resultant diagonal form of $\hat{\mathcal{H}}$ is

$$
\hat{\mathcal{H}}=\tilde{E}_{0}+\sum_{\mathbf{k}}\left[\left(\omega_{\mathbf{k}}^{0}-h\right) \beta_{\mathbf{k} \uparrow}^{\dagger} \beta_{\mathbf{k} \uparrow}+\left(\omega_{\mathbf{k}}^{0}+h\right) \beta_{\mathbf{k} \downarrow}^{\dagger} \beta_{\mathbf{k} \downarrow}\right] .
$$

Thus, the low-energy spectrum for $h<h_{c 1}$ is

$$
\tilde{\omega}_{\mathbf{k}}^{<} \equiv \omega_{\mathbf{k}}^{0}-h=\sqrt{\mu^{2}+2 \mu s^{2} \epsilon_{\mathbf{k}}}-h .
$$

The band $\tilde{\omega}_{\mathbf{k}}^{<}$has a minimum at the antiferromagnetic wave vector $\mathbf{Q}=(\pi, \pi, \pi)$ with the gap $\Delta^{<}=\omega_{\mathbf{Q}}^{0}-h$, whose vanishing point defines the critical field $h_{c 1}=g \mu_{B} H_{c 1}=$ $\omega_{\mathbf{Q}}^{0}$. The ground state energy is also affected by quantum fluctuations,

$$
\tilde{E}_{0}=E_{0}+\sum_{\mathbf{k}}\left(\omega_{\mathbf{k}}^{0}-\mu-s^{2} \epsilon_{\mathbf{k}}\right)
$$

The saddle point conditions $\partial \tilde{E}_{0} / \partial s=\partial \tilde{E}_{0} / \partial \mu=0$ lead to the self-consistent equations for the parameters $s$ and $\mu$,

$$
s^{2}=2-\frac{1}{N} \sum_{\mathbf{k}} \frac{\mu+s^{2} \epsilon_{\mathbf{k}}}{\omega_{\mathbf{k}}^{0}}, \quad D=\mu+\frac{\mu}{N} \sum_{\mathbf{k}} \frac{\epsilon_{\mathbf{k}}}{\omega_{\mathbf{k}}^{0}} .
$$

Using the Hamiltonian parameters for DTN given by Zvyagin et al. (2007), the resulting values are $s^{2}=0.92$ and $\mu=10.3 \mathrm{~K}$. The value of $s^{2}$ corresponds to a $\sim 10 \%$ correction relative to the mean-field solution (56) and it implies that quantum fluctuations are rather strong. This is indeed expected for a quasi-one-dimensional magnet like DTN. By introducing the Lagrange multiplier $\mu$, we allow $\left\langle b_{0 \mathbf{r}^{\prime}}^{(\dagger)}\right\rangle=s$ to deviate significantly from one in presence of strong quantum fluctuations. This is important because zeropoint fluctuations contribute to the stabilization of the $T=0$ quantum paramagnet. Indeed, the introduction of the Lagrange multiplier is necessary to reproduce the value of $h_{c 1}$ that is obtained from controlled quantum Monte Carlo simulations and that is also experimentally observed. On the other hand, the Holstein-Primakoff approach restricts $\left\langle b_{0 \mathbf{r}^{\prime}}^{(\dagger)}\right\rangle$ to be much closer to 1 and incorrectly predicts a magnetically ordered $H=0$ state. However, the main disadvantage of introducing the Lagrange multiplier is that the resulting approach is no longer a $1 / N \mathcal{S}$ expansion (only a subset of higher-order terms in $1 / N \mathcal{S}$ is included). The main negative consequence is the lack of a gapless Goldstone mode that must exist in the magnetically ordered state above $h_{c 1}$. In other words, if we introduce the Lagrange multiplier to describe the fluctuations around the magnetically ordered mean-field state for $h>h_{c 1}$, we obtain that the low-energy spectrum of excitations is gapped. Consequently, the continuous quantum phase transition from the quantum paramagnet to the canted $X Y$ antiferromagnet (BEC QCP) is not captured by this approximation, while it is correctly described within the Holstein-Primakoff scheme (1/NS expansion).

\section{Dilute gas}

As far as we know, Batyev and Braginskii (1984) were the first to exploit the analogy between a spin system and a Bose gas in order to apply the well-developed dilute gas approximation (Beliaev, 1958) to an antiferromagnet near the saturation field. We have seen in Sec. II.A that a spin $S=1 / 2$ antiferromagnet near the saturation field can be mapped into a dilute gas of hard-core bosons. The hard-core constraint can be replaced by an infinitely repulsive on-site interaction that has to be added to the off-site interaction potential (which arose from the Ising terms of the exchange interactions). This mapping has been described in detail in Sec. II.A [see Eq. (9)]. The goal of this section is to review the dilute gas approach near the field-induced QCP and briefly describe the successful applications of this approach to several quantum magnets. As mentioned, this is one of the very few controlled approaches for magnetic systems that are near the quantum limit. In particular, it is useful for determining the nature of the 
magnetically ordered state of frustrated quantum magnets near the saturation field (Nikuni and Shiba, 1995).

In Sec. II.D.1 we derived an effective model for hard-core bosons by keeping the two lowest-energy eigenstates of each unit cell. In this description, the low-field quantum paramagnet corresponds to the vacuum of hard-core bosons, while the magnetically ordered state right above $H_{c 1}$ corresponds to a condensate of a dilute gas of hard-core bosons. The general form of this effective Hamiltonian is

$$
\mathcal{H}=\sum_{\mathbf{q}}\left(\epsilon_{\mathbf{q}}-\mu\right) b_{\mathbf{q}}^{\dagger} b_{\mathbf{q}}+\frac{1}{2 N} \sum_{\mathbf{q}, \mathbf{k}, \mathbf{k}^{\prime}} V_{\mathbf{q}} b_{\mathbf{k}}^{\dagger} b_{\mathbf{k}^{\prime}}^{\dagger} b_{\mathbf{k}^{\prime}-\mathbf{q}} b_{\mathbf{k}+\mathbf{q}},
$$

where $\epsilon_{\mathbf{Q}_{j}}=0$ and $\mathbf{Q}_{j}$ is a wave vector that minimizes $\epsilon_{\mathbf{q}}$ $(1 \leq j \leq M)$. In principle, there can be more than one wave vector $\mathbf{Q}_{j}$ that minimizes $\epsilon_{\mathbf{q}}$. This is indeed the case for highly frustrated antiferromagnets such as the $X X Z$ model on the triangular lattice (Nikuni and Shiba, 1995) for which $M=2$. $M=1$ in absence of frustration, implying that the bosons must condense in a single wave vector state. This is for instance the case of the the antiferromagnetic $X X Z$ model on a cubic lattice in which the bosons condense at $\mathbf{Q}=(\pi, \pi, \pi)$ leading to a canted $X Y$ AFM phase in terms of the original spin language. The situation is less clear for frustrated systems with $M>1$ because the bosons can condense in a single-particle state that is an arbitrary linear combination of the $M$ different $\mathbf{Q}_{j}$ states. All of these states are degenerate at the noninteracting level. In these cases, the interaction term of Eq. (63) plays a more important role because it determines the optimal linear combination that minimizes the repulsion between bosons. Therefore, we need a controlled approach for treating the interaction terms. Semiclassical approaches do not include the full effect of quantum fluctuations and consequently can lead to an incorrect ordered state in the quantum limit that we are currently considering. Fortunately, such a controlled approach exists and was originally introduced by Beliaev in the 1950s (Beliaev, 1958). We note that the approach was introduced for bosonic gases that usually have a unique single-particle ground state. However, it can be easily extended to degenerate situations, such as the ones presented by frustrated spin magnets, for which this formalism has a much bigger impact (Stone et al., 2008a; Samulon et al., 2010). The advantage of treating a spin system as a gas of bosons becomes very clear at this point.

The hard-core constraint is imposed by an on-site repulsion $U \rightarrow \infty$. The bare four-point boson vertex is given by $V_{\mathbf{q}}=U+\gamma_{\mathbf{q}}$. The Bethe-Salpeter equation for the scattering function with zero total frequency is

$$
\Gamma_{\mathbf{q}}\left(\mathbf{k}, \mathbf{k}^{\prime}\right)=V_{\mathbf{q}}-\int_{-\pi}^{\pi} \frac{d^{3} p}{8 \pi^{3}} V_{\mathbf{q}-\mathbf{p}} \frac{\Gamma_{\mathbf{p}}\left(\mathbf{k}, \mathbf{k}^{\prime}\right)}{\epsilon_{\mathbf{k}+\mathbf{p}}+\epsilon_{\mathbf{k}^{\prime}-\mathbf{p}}}
$$

This corresponds to the exact solution of the two-particle problem and consequently provides an effective interaction vertex that is asymptotically exact for $\rho \rightarrow 0$ [see Abrikosov, Gorkov, and Dzyaloshinski (1975); and Fetter and Walecka (2003)]. In terms of Feynman diagrams, Eq. (64) corresponds to adding all the ladder diagrams shown in Fig. 4 that give the dominant contribution in an expansion in $a \rho^{1 / d}$, where $a$ is the

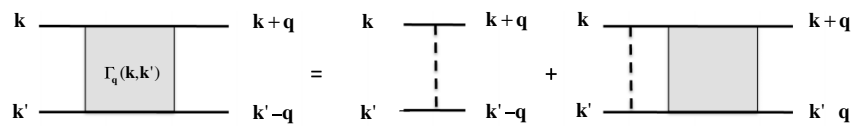

FIG. 4 (color online). Diagrammatic representation of Eq. (64). The ladder diagrams give the dominant contribution in an expansion in $a \rho^{1 / d}$, where $a$ is the scattering length of the interaction potential $V_{\mathbf{q}}$.

scattering length of the interaction potential $V_{\mathbf{q}}$ and $a \rho^{1 / d}$ is the so-called lattice gas parameter.

By taking the limit $U \rightarrow \infty$ and integrating both sides of Eq. (64), we obtain

$$
\begin{aligned}
\langle\Gamma\rangle\left(\mathbf{k}, \mathbf{k}^{\prime}\right) & =\int_{-\pi}^{\pi} \frac{d^{3} p}{8 \pi^{3}} \Gamma_{\mathbf{p}}\left(\mathbf{k}, \mathbf{k}^{\prime}\right) \\
& =U\left[1-\int_{-\pi}^{\pi} \frac{d^{3} p}{8 \pi^{3}} \frac{\Gamma_{\mathbf{p}}\left(\mathbf{k}, \mathbf{k}^{\prime}\right)}{\epsilon_{\mathbf{k}+\mathbf{p}}+\epsilon_{\mathbf{k}^{\prime}-\mathbf{p}}}\right] .
\end{aligned}
$$

By replacing this expression into Eq. (64) and taking the $U \rightarrow \infty$ limit in (65), we obtain the system of equations

$$
\begin{aligned}
\Gamma_{\mathbf{q}}\left(\mathbf{k}, \mathbf{k}^{\prime}\right) & =\gamma_{\mathbf{q}}+\langle\Gamma\rangle\left(\mathbf{k}, \mathbf{k}^{\prime}\right)-\int_{-\pi}^{\pi} \frac{d^{3} p}{8 \pi^{3}} \gamma_{\mathbf{q}-\mathbf{p}} \frac{\Gamma_{\mathbf{p}}\left(\mathbf{k}, \mathbf{k}^{\prime}\right)}{\epsilon_{\mathbf{k}+\mathbf{p}}+\epsilon_{\mathbf{k}^{\prime}-\mathbf{p}}} \\
1 & =\int_{-\pi}^{\pi} \frac{d^{3} p}{8 \pi^{3}} \frac{\Gamma_{\mathbf{p}}\left(\mathbf{k}, \mathbf{k}^{\prime}\right)}{\epsilon_{\mathbf{k}+\mathbf{p}}+\epsilon_{\mathbf{k}^{\prime}-\mathbf{p}}}
\end{aligned}
$$

where we have used $\left\langle\gamma_{\mathbf{p}}\right\rangle=0$. This system of integral equations can be reduced to a linear system of algebraic equations by expanding $\Gamma_{\mathbf{q}}\left(\mathbf{k}, \mathbf{k}^{\prime}\right)$ in lattice harmonics of the wave vector q. Examples of this expansion for particular lattices can be found in Batyev and Braginskii (1984) and Nikuni and Shiba (1995).

The next step is to take the long-wave length limit of Eq. (63) by expanding around each of the wave vectors $\mathbf{Q}_{j}$ that minimizes the single-particle dispersion,

$$
\begin{aligned}
\mathcal{H}_{\mathrm{eff}}= & \sum_{\mathbf{q} ; j=1, M ; \nu}\left(\frac{q_{\nu}^{2}}{2 m_{j}^{\nu}}-\mu\right) b_{\mathbf{Q}_{j}+\mathbf{q}}^{\dagger} b_{\mathbf{Q}_{j}+\mathbf{q}} \\
& +\frac{1}{2 N} \sum_{q, k, k^{\prime} ; j, j^{\prime}, l, l^{\prime}} \Gamma_{\mathbf{Q}_{l}-\mathbf{Q}_{j}}\left(\mathbf{Q}_{j}, \mathbf{Q}_{j^{\prime}}\right) b_{\mathbf{Q}_{j}+\mathbf{k}}^{\dagger} b_{\mathbf{Q}_{j^{\prime}}+k^{\prime}}^{\dagger} \\
& \times b_{\mathbf{Q}_{l^{\prime}+\mathbf{k}^{\prime}-\mathbf{q}}} b_{\mathbf{Q}_{l}+\mathbf{k}+\mathbf{q}} \delta\left(\mathbf{Q}_{j}+\mathbf{Q}_{j^{\prime}}-\mathbf{Q}_{l}-\mathbf{Q}_{l^{\prime}}-\mathbf{G}\right),
\end{aligned}
$$

where $\mathbf{G}$ is a reciprocal lattice vector, $\nu=\{x, y, z\}$, and $q, k, k^{\prime} \ll 1$. The effective masses $m_{j}^{\nu}$ are given by

$$
\frac{1}{m_{j}^{\nu}}=\left.\frac{\partial^{2} \epsilon_{\mathbf{q}}}{\partial q_{\nu}^{2}}\right|_{\mathbf{q}=\mathbf{Q}_{j}}
$$

Here we assumed that the mass tensor for each $\mathbf{Q}_{j}$ is diagonal in order to simplify the expression of $\mathcal{H}_{\text {eff }}$. The optimal magnetic ordering right above $H_{c 1}$ is obtained from a meanfield variational treatment of the interaction term of $\mathcal{H}_{\text {eff }}$. The corresponding variational parameters consist of one amplitude and one phase for each $\mathbf{Q}_{j}:\left\langle b_{\mathbf{Q}_{j}}^{\dagger}\right\rangle=A_{j} e^{i \phi_{j}}$. The optimal 
magnetic ordering is then determined by the relative magnitude of the effective interactions vertices $\Gamma_{\mathbf{Q}_{l}-\mathbf{Q}_{j}}\left(\mathbf{Q}_{j}, \mathbf{Q}_{j^{\prime}}\right)$. By diagonalizing the resulting quadratic mean-field Hamiltonian it is possible to obtain the low energy excitations and compute different thermodynamic quantities near the field-induced QCP. This procedure has been successfully applied to a long list of quantum magnets including $\mathrm{TlCuCl}_{3}$ (Misguich and Oshikawa, 2004; Sirker, Weiße, and Sushkov, 2004), $\mathrm{BaCuSi}_{2} \mathrm{O}_{2}$ (Batista et al., 2007; Schmalian and Batista, 2008), DTN (Kohama et al., 2011; Weickert et al., 2012), and $\mathrm{Ba}_{3} \mathrm{Mn}_{2} \mathrm{O}_{8}$ (Samulon et al., 2009, 2010; Suh et al., 2011; Kamiya and Batista, 2013).

In general, there are no controlled analytical techniques for solving the problem far away from the dilute limit. The $d=1$ case is an exception because one can still apply the bosonization technique even for high concentrations of bosons (Giamarchi, 2004). For spin-ladder compounds like $\mathrm{TlCuCl}_{3}$, one can combine the bosonization method (to compute the single-ladder susceptibility) with the random phase approximation (RPA) to include the small interladder coupling. Thus it is possible to obtain the thermodynamic phase diagram of weakly coupled ladder compounds (Bouillot et al., 2011). Unfortunately, this approach is no longer valid for $d=2,3$ systems that have similar couplings along the different dimensions. In these cases it is necessary to implement controlled numerical techniques to provide an accurate or quantitative description of the properties that are typically measured in quantum magnets.

\section{Numerical approaches}

The analytical approaches that we considered in the previous subsections are useful as long as certain control parameters remain small enough to guarantee the validity of the corresponding approximations. For instance, the dilute gas approach gives quantitatively accurate results for low enough boson concentrations. However, this ceases to be true if we move far enough away from the QCP. Fluctuations induced by higher-order diagrams in the lattice gas parameter have to be included in order to obtain a quantitatively accurate description of this nonuniversal regime. The generalized spin-wave approach is based on a mean-field approximation which assumes that quantum fluctuations are small relative to the average value of the order parameter. However, this assumption is typically not correct for low-dimensional or highly frustrated quantum magnets. Therefore, in the absence of a small parameter that justifies the validity of our analytical approach, it is convenient to use a controlled numerical technique to complement the analytical results. Quantitatively accurate estimations of the full $(H, T)$ phase diagram and different thermodynamic properties are particularly useful to determine the validity of the model under consideration.

There are two types of numerical approaches that are typically applied to the study of quantum magnets: quantum Monte Carlo (QMC) simulations and density matrix renormalization group (DMRG) calculations (White, 1992). QMC methods (Prokofev, Svistunov, and Tupitsyn, 1998; Sandvik, 1999; Syljuåsen and Sandvik, 2002; Kawashima and Harada, 2004) allow for computing static correlation functions of models for interacting quantum spin systems in any spatial dimension. The main limitation of the QMC techniques is that they are limited to systems that do not include off-diagonal frustrating interactions such as frustrated hopping terms in the case of lattice gases or frustrated $X Y$ interaction terms in the case of quantum spin systems. There are some particular Hamiltonians that include off-diagonal frustration but their low-energy effective model is not frustrated. This is the case of the Hamiltonian given in Eq. (25). Although the interdimer exchange interactions are always frustrated, there is a region of exchange parameters for which the sign of the hopping amplitudes $t_{\mathbf{r}, \mathbf{r}^{\prime}}$ of the effective Hamiltonian remains negative [see Eq. (31)]. In cases like this one, one can still use a QMC approach for simulating the effective low-energy model. Another important limitation of QMC methods is the difficulty of computing dynamical correlation functions.

Controlled QMC calculations of different thermodynamic quantities away from a QCP are important for the experimental determination of critical exponents. In the first place, it is necessary to determine the window of parameters, such as temperature and magnetic field, where the universal powerlaw behaviors are dominant. In other words, there is always a crossover temperature $T_{U}$ above which corrections to scaling become important. A clear example of this situation is illustrated by the early measurements of the critical exponent associated with the phase boundary $H_{c 1}(T)$ that divides the paramagnetic and field-induced canted $X Y$ AFM phases of several quantum paramagnets,

$$
H_{c 1}(T)-H_{c 1}(0) \propto T^{\phi}
$$

for low enough temperature. According to our Eq. (24), $\phi=d / z$ for a BEC QCP. The question is: how low is "low enough"? Early fits of experimental data over arbitrary windows of temperature led to quite unexpected values of the exponent $\phi$. For instance, values like $\phi=1.7$ (1) (Oosawa, Ishii, and Tanaka, 1999), $\phi=2.2(1)$ (Nikuni et al., 2000), $\phi=2.0(1)$ (Tanaka et al., 2001), $\phi=2.3(1)$ (Oosawa et al., 2002), and $\phi=1.67(1)$ (Shindo and Tanaka, 2004) were reported by early experiments in the same compound, $\mathrm{TlCuCl}_{3}$, while $\phi=2.6(2)$ (Paduan-Filho, Gratens, and N. F. Oliveira, 2004a; Paduan-Filho, Gratens, and Oliveira, 2004b) was reported for DTN. All of these estimates are higher than the mean-field exponent $\phi=3 / 2$ that is expected for $d=3(D=d+z=5)$. The origin of this large discrepancy was explained by Nohadani et al. (2004) and Kawashima (2005) who performed large-scale QMC simulations for computing the thermodynamic phase boundary. Nohadani et al. (2004) studied a 3D Heisenberg model of coupled $S=$ $1 / 2$ dimers and showed that the exponent $\phi$ approaches the expected value of $3 / 2$ when the window of temperatures is reduced. Kawashima computed the phase boundary $H_{c 1}(T)$ of the $S=1 / 2 X Y$ model [see Eq. (6)] in $d=3$. A scaling analysis of his numerical results shows that the expected exponent of $\phi=3 / 2$ gives the best fit in a low-temperature window of $T<\simeq 0.4 T_{c \max }$, where $T_{c \max }$ is the maximum magnetic ordering temperature as a function of the applied field. However, an exponent $\phi=2$ gives the best fit if we try to fit the phase boundary with the power law (69) over a temperature window of $0.7 T_{c \max }$. This example illustrates the importance of complementing analytical calculations with 
careful numerical simulations of the model under consideration. Large-scale QMC simulations of a 3D Heisenberg model of coupled spin-1/2 dimers also confirmed the expected exponents for the field dependence of the staggered (order parameter) and uniform magnetization (Nohadani, Wessel, and Haas, 2005). QMC simulations have been useful for obtaining the phase boundaries and several thermodynamic properties of $\mathrm{BaCuSi}_{2} \mathrm{O}_{2}$ (Jaime et al., 2004; Sebastian et al., 2005; Batista et al., 2007), $\mathrm{NiCl}_{2}-4 \mathrm{SC}\left(\mathrm{NH}_{2}\right)_{2}$ (DTN) (Zapf et al., 2006, 2008; Zvyagin et al., 2007; Kohama et al., 2011), and (HPIP) $)_{2}-\mathrm{CuBr}_{4}$ (Thielemann, Rüegg, Kiefer et al., 2009; Bouillot et al., 2011). These calculations were essential for validating the spin models that describe these quantum magnets.

The second method that has been applied to the description of many quantum paramagnets in a magnetic field is the DMRG (White, 1992). This method is appropriate for onedimensional systems and it is not limited to nonfrustrated models. It is particularly useful for describing quantum paramagnets comprising weakly coupled frustrated ladders. Although the method is mainly adequate for one-dimensional systems such as a single ladder, this alone can provide valuable information if the interladder exchange coupling is weak enough. This method has evolved to the extent that dynamical properties such as the frequency and momentum dependence of the magnetic structure factor can be computed very accurately (Kühner and White, 1999). The DMRG method has also been recently applied to the computation of the field-induced magnetization curve of $\mathrm{SrCu}_{2}\left(\mathrm{BO}_{3}\right)_{2}$ (Moukouri, 2008; Manmana et al., 2011; Jaime et al., 2012), a 2D frustrated paramagnet whose field-induced magnetization plateaus have been the subject of intensive research during the last fifteen years. The recent work by Bouillot et al. (2011) provides a clear example of combination of state of the art numerical techniques for computing static and dynamical properties of weakly coupled ladders. The work is motivated by recent experiments on the compound (HPIP $)_{2}-\mathrm{CuBr}_{4}$. While single-ladder static and dynamical properties are computed with the DMRG technique, the thermodynamic phase diagram for weakly coupled ladders is obtained from QMC simulations.

\section{E. Theoretical summary}

Here we summarize the key points of the theory section, and the minimum conditions for observing BEC-like behavior in magnetic systems. The most important prerequisite for observing BEC in quantum magnets is boson number conservation. This corresponds to uniaxial symmetry of the magnetic spin Hamiltonian. The number of bosons in these magnetic realizations is the total magnetization $M_{z}$ along the field direction, while the number of condensed particles is proportional to the square of the ordered transverse component ( $X Y$ staggered magnetization in the case of simple antiferromagnets). Since $M_{z}$ is the infinitesimal generator of global spin rotations along the $z$ axis, the Hamiltonian is invariant under finite global spin rotations along the same axis if it commutes with $M_{z}$ [U(1) symmetry]. As we saw in Sec. II.B, the uniaxial symmetry can be broken in real quantum magnets by the effective spin-spin interactions induced by spin-orbit coupling or dipole-dipole interactions. These symmetry-breaking terms can be small enough to be neglected at temperatures above tens of millikelvins. However, the small symmetry-breaking interactions will ensure that $M_{z}$ will fluctuate on short time scales due to terms in the Hamiltonian that connect states of different $M_{z}$. Thus, supercurrents and other metastable excited states will relax in these systems.

To minimize spin-orbit couplings that tend to pin spins along certain lattice directions, lattices can be chosen with greater than twofold symmetry, e.g., fourfold or sixfold in a plane. If present, DM interactions are normally the strongest uniaxial-symmetry-breaking terms. However, these terms can be avoided by choosing crystals with high enough symmetry (Moriya, 1960). Dipole-dipole interactions will also break uniaxial symmetry. These are unavoidable, but their influence can be reduced by selecting certain classes of magnets. For instance, uniaxial symmetry-breaking terms of spin dimer systems can be minimized by choosing compounds where the dimers are parallel to each other and the interdimer distance $a$ is significantly larger than the distance $l_{D}$ between the two magnetic ions in the same dimer. If the applied magnetic field is parallel to the dimers, then the intradimer dipolar interaction does not break the U(1) symmetry, while the already weak interdimer dipolar coupling is further reduced at low energies (singlet-triplet subspace) by a prefactor of order $l_{D} / a$. This observation could explain why most of the quantum magnets that have been used for studying BEC-related phenomena consist of weakly coupled spin dimers.

Once the uniaxial symmetry is established, down to some well-characterized energy scale, we must apply the magnetic field along the symmetry axis. Any magnetic realization of BEC-like physics must have at least two low-energy levels per unit cell with different magnetization (eigenvalue of $S^{z}$ ). We can assign each spin level to a different boson occupation. The raising and lowering spin operators are mapped into creation and annihilation bosonic operators that satisfy the same commutation relations. Note that spin operators from different ions commute with each other. ${ }^{1}$ The conservation of the total magnetization $M_{z}$ implies conservation of the particle number while the applied magnetic field $H_{z}$ acts as a chemical potential that tunes the boson number. The ground state of a quantum paramagnet is the empty state in the bosonic language and there is a gap for creating a single boson excitation (e.g., the gap between the spin levels). The application of a magnetic field closes the gap at $H=H_{c 1}$ and populates the ground state with bosons. In the dilute limit, the bosons normally condense into a BEC state. The BEC state corresponds to $X Y$ magnetic ordering in the spin language and this phase extends up to a second critical field $H_{c 2}$ if the kinetic energy dominates over the off-site interactions between bosons. While the ordered $(X Y)$ spin component is perpendicular to the applied field and corresponds to

\footnotetext{
${ }^{1}$ It is important to note that this mapping connects only the (bosonic) spin degrees of freedom of a system of localized electrons (the orbital degree of freedom is quenched) with a gas of spinless bosons. Therefore, the mapping does not violate the spin-statistics theorem.
} 
the density of condensed bosons, there is also a uniform spin component along the field direction (canted $X Y$ ordering) that corresponds to the overall boson density.

We note that for two-level systems, bosons are subject to the constraint of a hard-core repulsion, e.g., a maximum of one boson can occupy each effective orbital. This constraint is essential to guarantee that a two-level system is mapped into another two-level system (the mapping must not change the dimension of the Hilbert space). In a mean-field description, each two-level system is in a linear superposition of the two states with different magnetization, i.e., a linear superposition of states with zero and one boson. As the magnetic field increases, the moments become more polarized along the field direction and lose their $x y$ component. The ordered moment is finally suppressed at the second critical point $H=H_{c 2}$, where the spins become fully polarized. Above this second critical field, the ground state contains one hard-core boson in each site and can be regarded as a Mott insulator in the bosonic language.

The two QCPs at $H_{c 1}$ and $H_{c 2}$ belong to the BEC universality class in dimension $D=d+z$. The dynamical exponent is $z=2$ because the single-boson dispersion is quadratic at the BEC QCPs: $\omega \propto k^{z}$ for $k \ll 1$ with $z=2$. This quadratic dispersion is a direct consequence of the fact that the driving parameter (magnetic field) couples to a conserved quantity (total magnetization $M_{z}$ ): the quadratic shape of the single-particle excitations of the quantum paramagnet cannot be modified when $H$ approaches $H_{c 1}$ because these excitations have the same $S^{z}$ eigenvalue. Therefore, the only effect of the applied field is to close the gap while keeping the dispersion unaltered all the way up to $H_{c 1} \cdot M_{x y}$ is zero outside of the range $H_{c 1}<H<H_{c 2}$. Inside that interval it is dome shaped, reaching a peak somewhere between the two critical fields. The $X Y$ ordering requires a spontaneous symmetry breaking that chooses both a size and an orientation of the moments, i.e., the order parameter is a two-dimensional vector. Thus, this ordering can be suppressed either by suppressing the amplitude of the order parameter or by increasing its phase fluctuations. BEC-like transitions correspond to the first case where the amplitude is suppressed, while transitions induced by phase fluctuations lead to the so-called $\mathrm{O}(2)$ universality class that has a dynamical exponent $z=1$. The critical exponents expected for the BEC QCP are summarized in Table III. The dispersion

TABLE III. Temperature dependencies of the thermodynamic quantities: Phase boundary, magnetization $M(T)$, thermal expansion $\Delta L / L, \alpha(T)$, specific heat $C(T)$, thermal Grüneisen parameter $\Gamma(T)$, and magnetic Grüneisen parameter $\Gamma_{\text {mag }}(T)$ at the field-induced QCP at $H=H_{c}$ as the temperature $T \rightarrow 0$. The variable $d$ denotes the spatial dimensionality of the system. The exponents of the Ising-like $\mathrm{QCP}$ are given for $d=3$.

\begin{tabular}{lcc}
\hline \hline Property & $X Y$ AFM order & Ising (3D) \\
\hline Phase boundary $H_{c}(T)-H_{c}(0)$ & $T^{d / 2}, \nu=2 / \mathrm{d}$ & $T^{2}$ \\
Magnetization $M\left(H_{c}, T\right)$ & $T^{d / 2}$ & $T^{2}$ \\
Thermal expansion $\frac{\Delta L}{L}\left(H_{c}, T\right)$ & $T^{d / 2}$ & $T^{2}$ \\
Coefficient of thermal & $T^{(d / 2)-1}$ & $T$ \\
$\quad$ expansion $\alpha\left(H_{c}, T\right)$ & & \\
Specific heat $C\left(H_{c}, T\right)$ & $T^{d / 2}$ & $T^{3}$ \\
Grüneisen parameter $\Gamma \propto \alpha / C$ & $T^{-1}$ & $T^{-2}$ \\
Magnetic Grüneisen $\Gamma_{\text {mag }} \propto \alpha / M$ & $T^{-1}$ & $T^{-2}$ \\
\hline \hline
\end{tabular}

relation of the bononic quasiparticles (magnons in the spin language) becomes linear, $\omega \propto k$ for $k \ll 1$, in the ordered state that exists between $H_{c 1}$ and $H_{c 2}$. This is the Goldstone mode that is expected from the spontaneous breaking of the $\mathrm{U}(1)$ symmetry.

\section{EXPERIMENTS}

\section{A. Compounds}

In the following we summarize experiments on quantum magnets that have been described in terms of BEC. The quantum magnets described in this section benefit from the fact that BEC behavior can be accessed for values of the tuning parameters, such as temperature, magnetic field, and pressure, that are readily accessible in many condensed matter physics laboratories (see Fig. 5). Indeed, Table I shows magnetic ordering temperatures compatible with standard liquid ${ }^{4} \mathrm{He}$ as well as ${ }^{3} \mathrm{He}-{ }^{4} \mathrm{He}$ dilution refrigerators, when magnetic fields produced by either superconducting, resistive, resistive hybrid, or resistive pulsed magnets are applied. Simultaneously, the relatively small exchange interactions that make these systems amenable to liquid helium studies can be tuned as a function of laboratory-produced external pressures.

As we saw in the previous sections, the mapping between spins and bosons requires at least two different low-energy spin levels. Then, each spin level corresponds to a different boson occupation and the gap between these levels can be tuned with magnetic field. In most quantum magnets exhibiting BEC, a nonmagnetic ground state at zero magnetic field is separated from a magnetic excited state by a gap $\Delta$. This gap can be tuned to zero by applied magnetic fields, resulting in a magnetic-field-induced QCP at $H_{c 1}$ that belongs to the BEC universality class.

There are several different ways to create the zero-field gap. The most widely studied method involves $S=1 / 2$ dimers, and has been investigated in $\mathrm{BaCuSi}_{2} \mathrm{O}_{6}, \mathrm{TlCuCl}_{3}$, $\mathrm{KCuCl}_{3}, \mathrm{~Pb}_{2} \mathrm{~V}_{3} \mathrm{O}_{9}, \mathrm{Ba}_{3} \mathrm{Cr}_{2} \mathrm{O}_{8}, \mathrm{Sr}_{3} \mathrm{Cr}_{2} \mathrm{O}_{8},(\mathrm{CuCl}) \mathrm{LaNb}_{2} \mathrm{O}_{7}$, Sul- $\mathrm{Cu}_{2} \mathrm{Cl}_{4}$, and PHCC among others (see Table I). In these spin dimer materials, two closely coupled $S=1 / 2$ spins form a dimer with antiferromagnetic coupling $J_{0}$. Thus, the

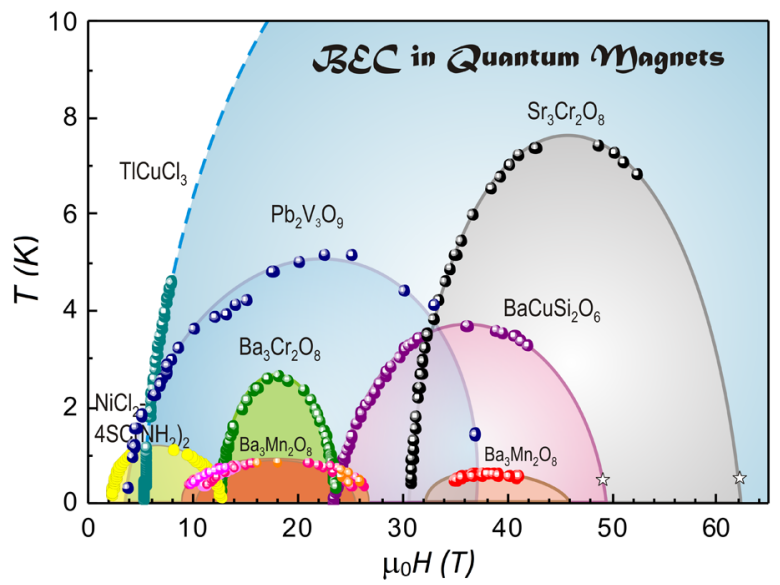

FIG. 5 (color online). $\quad(T, H)$ phase diagram for several quantum magnets studied in the context of BEC. 
single-dimer ground state is a singlet and the lowest-energy excitation is an $S=1$ triplet consisting of $\left|S^{z}=-1\right\rangle$, $\left|S^{z}=0\right\rangle$, and $\left|S^{z}=-1\right\rangle$ states, which have an energy $J_{0}$ relative to the singlet. The presence of interdimer interactions disperses the triplet levels into a band of excitations and the spin gap of the coupled system is reduced relative to the single-dimer gap $J_{0}$. This is shown for $\mathrm{TlCuCl}_{3}$ in Fig. 6. The branch of $\left|S^{z}=1\right\rangle$ excitations decreases linearly in magnetic field via the Zeeman effect, thereby creating a degeneracy between the lowest energy $\left|S^{z}=1\right\rangle$ excitation and the singlet ground state that leads to a divergent susceptibility at the wave vectors that minimize the triplet dispersion relation. Long-range magnetic order occurs between $H_{c 1}$ and $H_{c 2}$ and the spins align with the magnetic field above $H_{c 2}$ creating a saturated paramagnet.

Zero-field gaps also exist in systems of nondimerized $S=1$ spins. Single-ion anisotropy terms, like $D\left(S^{z}\right)^{2}$, can become important for $S \geq 1$ systems. One example of a system of $S=1$ spins and strong easy-plane single-ion anisotropy is $\mathrm{NiCl}_{2}-4 \mathrm{SC}\left(\mathrm{NH}_{2}\right)_{2}$ (DTN) (see Fig. 7). The $D\left(S^{z}\right)^{2}$ term $(D>0)$ splits the $S=1$ triplet of each $\mathrm{Ni}^{2+}$ ion into an $\mid S^{z}=$ $0\rangle$ ground state and a $\left|S^{z}= \pm 1\right\rangle$ doublet separated by a gap $D$. Unlike the isotropic $S=1 / 2$ dimer systems, the direction of the magnetic field is now important because the $D$ term already breaks the rotational $\mathrm{SU}(2)$ symmetry and so $\mathbf{H}$ must be applied along the direction of $D$ to retain uniaxial symmetry. As $\mathbf{H}$ angles away from $\hat{z}$, the BEC description becomes increasingly less valid and there is a crossover from a BEC QCP to an Ising QCP.

BEC has also been studied in systems of dimers with higher spin. For example, $\mathrm{Ba}_{3} \mathrm{Mn}_{2} \mathrm{O}_{8}$ and $\mathrm{F}_{2} \mathrm{PNNNO}$ are $S=1$ dimer compounds. In $\mathrm{Ba}_{3} \mathrm{Mn}_{2} \mathrm{O}_{8}$ (see Fig. 8), antiferromagnetic coupling of $S=1 \mathrm{Mn}$ spins within a dimer with strength $J_{0}$ produces an $|S=0\rangle$ singlet ground state, an $S=1$ spin triplet with a gap of $J_{0}$, and finally an $S=2$ spin quintuplet with a gap $3 J_{0}$ above the $|S=0\rangle$ ground state. As shown in Fig. 8, the energies of the $S^{z}=1$ triplet and $S^{z}=2$ quintuplet spin levels decrease linearly in magnetic field and lead to two domes of antiferromagnetic order, the first where the $S^{z}=1$ triplets condense, between $H_{c 1} \leq H \leq H_{c 2}$, and the second where the $S^{z}=2$ quintuplets condense, between $H_{c 3} \leq H \leq H_{c 4}$.

$\mathrm{Cs}_{2} \mathrm{CuCl}_{4}$ is an example of a compound that is already magnetically ordered in zero field (see Fig. 9), in contrast to all others discussed in this section, that have a zero-field gap. The BEC QCP is observed at the saturation field $H_{c}$ when the gap opens. The $\mathrm{Cu}^{2+} S=1 / 2$ moments form an anisotropic triangular lattice with two different antiferromagnetic couplings.

Tetramer or larger superstructures can also provide a route to BEC. Sul- $\mathrm{Cu}_{2} \mathrm{Cl}_{4}$ is such an example of a four-leg spin ladder compound. In another material, $(\mathrm{CuCl}) \mathrm{LaNb}_{2} \mathrm{O}_{7}$, it was initially thought that antiferromagnetically coupled tetramers could occur, resulting in a nonmagnetic collective ground state with a finite gap $\Delta$. Further studies found that an $S=1 / 2$ dimer description is more appropriate (Tsirlin and Rosner, 2010).

Finally, systems with infinite coupled chains can form gaps. For example, $\mathrm{IPA}-\mathrm{CuCl}_{3}$ is a Haldane system where effective $S=1$ spins are created by dimers of ferromagnetically

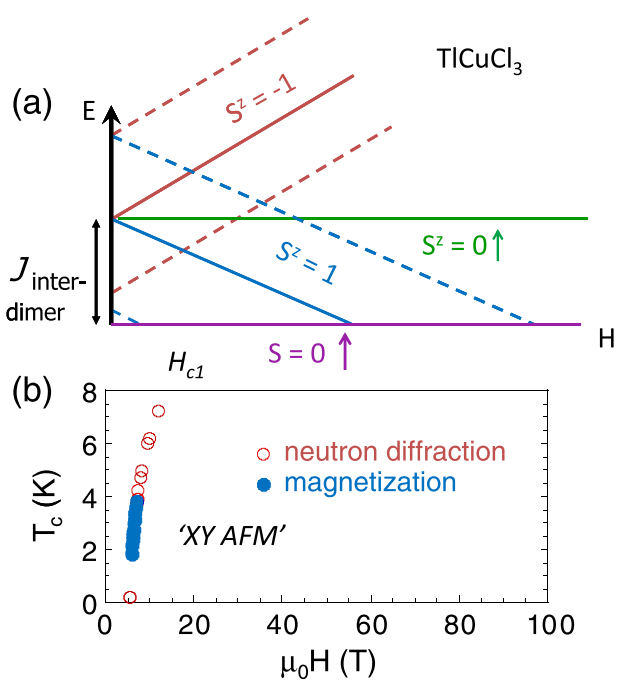

FIG. 6 (color online). (a) Cartoon of the spin levels of $\mathrm{TlCuCl}_{3}$ showing the zero-field gap due to the interdimer interactions $J_{\text {interdimer. }}$ The levels are dispersed, forming bands that evolve in magnetic fields due to the Zeeman interaction. Long-range order occurs in the region $H_{c 1}<H<H_{c 2}$. (b) Phase diagram of $\mathrm{TlCuCl}_{3}$ from elastic neutron diffraction (open symbols) and magnetization measurements (solid symbols). The complete phase diagram extends to approximately $100 \mathrm{~T}$, and the antiferromagnetically ordered phase occurs underneath and to the right of the data points. Adapted from Tanaka et al., 2001.

coupled $S=1 / 2$ spins. The effective $S=1$ moments are antiferromagnetically coupled along chains. While the interchain coupling is weak enough to observe a Haldane phase (quantum paramagnet) at zero field, it is sufficiently strong to create 3D magnetic ordering above a critical magnetic field $H_{c 1}$.

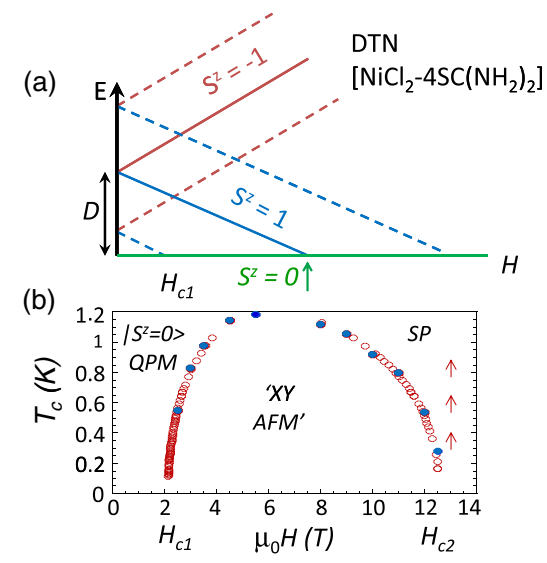

FIG. 7 (color online). (a) Cartoon of the spin levels of DTN $\left[\mathrm{NiCl}_{2}-4 \mathrm{SC}\left(\mathrm{NH}_{2}\right)_{2}\right]$ showing the $S^{z}= \pm 1$ excited doublet and $S^{z}=0$ ground state, separated by a zero-field gap $D$ due to single-ion anisotropy. As with Fig. 6, the spin levels are shown as bands due to magnetic dispersion. (b) Phase diagram from specific heat (solid symbols) and magnetocaloric effect (open symbols) showing a dome-shaped region of quasi- $X Y$ antiferromagnetic order (XYAFM), the low-field quantum paramagnet (QPM), and the high-field spin saturated phase (SP). Adapted from Zapf et al., 2006. 

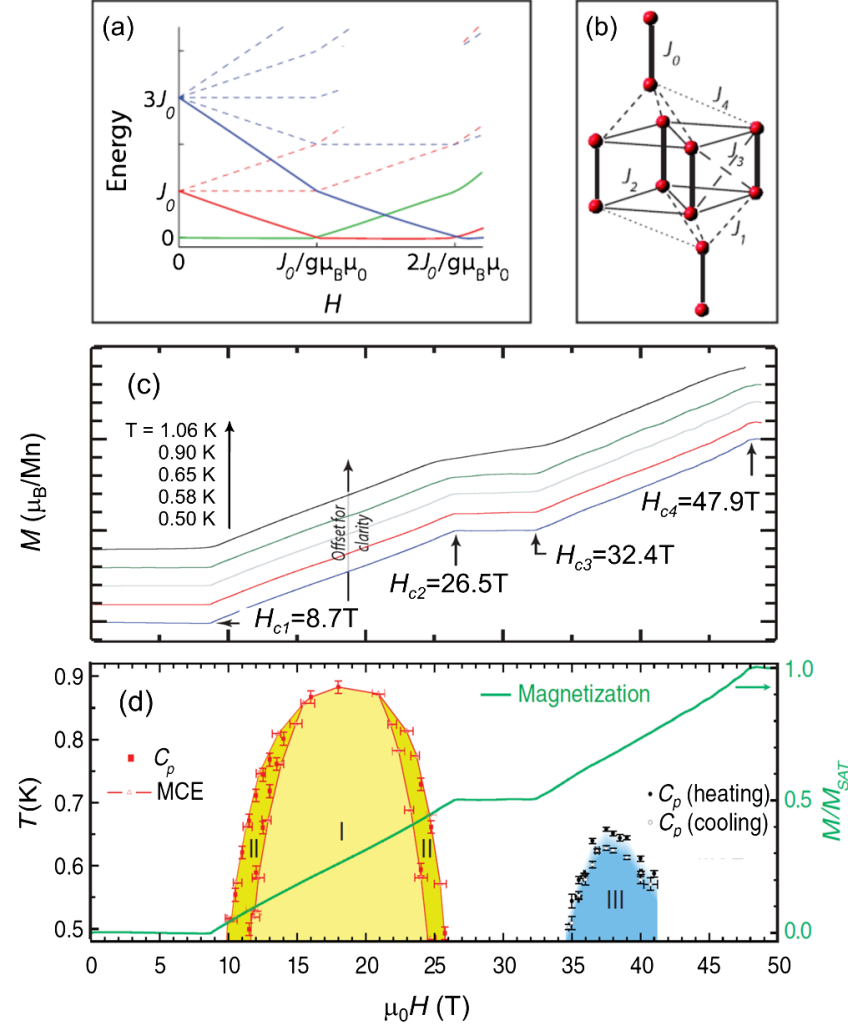

FIG. 8 (color online). The $S=1$ antiferromagnetically coupled dimer compound $\mathrm{Ba}_{3} \mathrm{Mn}_{2} \mathrm{O}_{8}$ forms an $S=0$ ground state, an $S=1$ excited triplet separated by the intradimer antiferromagnetic coupling $J_{0}$, and an $S=2$ quintuplet separated by $3 J_{0}$ from the ground state. (a) Evolution of the spin levels with applied magnetic field. (b) Schematic of the $S=1$ dimers in the lattice. (c) Magnetization $M$ vs magnetic field $H$ at various temperatures and (d) temperature-magnetic-field phase diagram determined from specific heat and magnetocaloric effect (MCE) showing two regions of $\mathrm{BEC}$, one where the lowest triplet level crosses the ground state, and one where the lowest quintuplet level crosses. The magnetization $M$ is overlaid. From Samulon et al., 2009.

\section{B. Measurements}

Experimental characterizations of BEC materials generally fall into three categories: (1) Determination of the $(T, H)$ phase diagram and estimation of the various parameters such as exchange interactions and zero-field splittings between spin levels. (2) Verification of uniaxial symmetry, e. g., using electron spin resonance and inelastic neutron scattering. (3) Determination of the power-law temperature dependences of thermodynamic properties like specific heat, magnetization, and the critical fields, and comparison to predictions for the BEC QCP.

In the literature reviewed here, the experimental characterizations of prospective quantum magnets typically start with a measurement of the magnetic susceptibility $(\chi)$ as a function of temperature to estimate the magnitude of the spin gap, or the lack of thereof. Some simple models can be used to assess the magnitude of exchange interactions from $\chi(T)$, although the results are very sensitive to the details of the model assumed. Measurements of magnetization $(M)$, specific
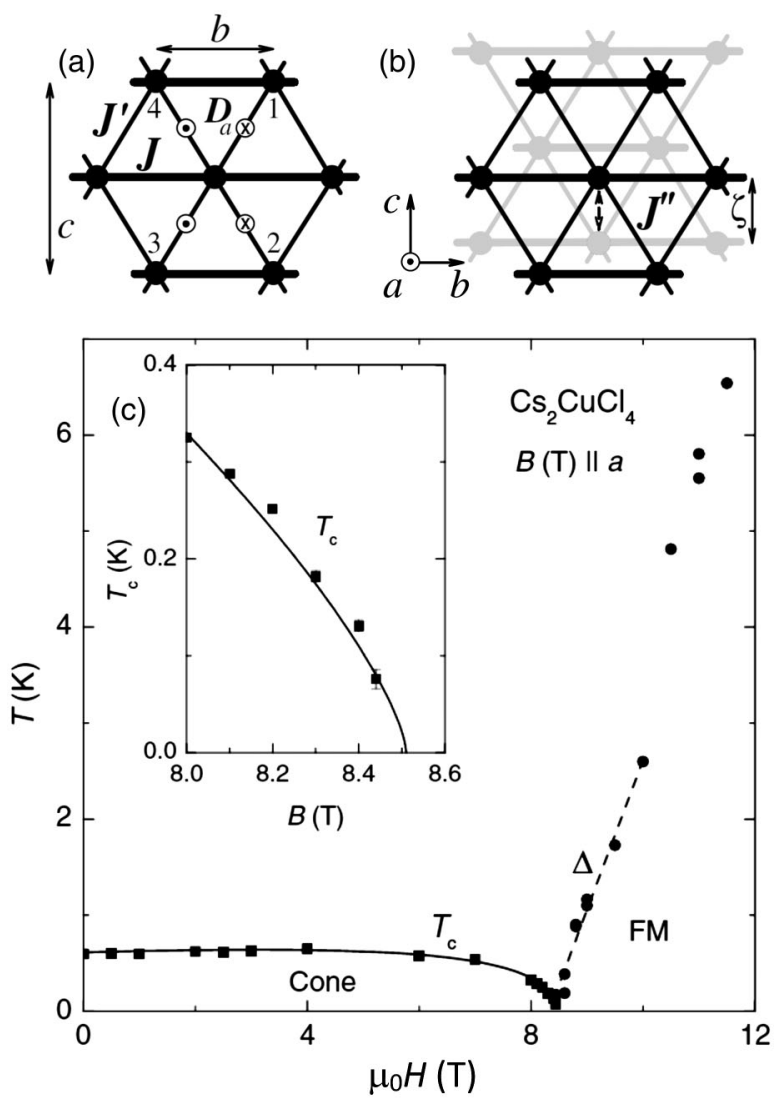

FIG. 9. (a),(b) Frustrated triangular arrangement of $S=1 / 2 \mathrm{Cu}$ spins in $\mathrm{Cs}_{2} \mathrm{CuCl}_{4}$ with couplings $J$ and $J^{\prime}$ in the $b-c$ plane and $J^{\prime \prime}$ between planes. Dzyaloshinskii-Moriya (DM) couplings $\mathbf{D}_{a}$ are also shown pointing into (dots) and out of (crosses) the $b c$ plane. (c) Temperature-magnetic-field phase diagram for magnetic fields along the $a$ axis showing a conelike magnetic state below the critical field $8.5 \mathrm{~T}$, and the opening of a gap above $8.5 \mathrm{~T}$ (Coldea et al., 2002; Radu et al., 2005).

heat $(C)$, thermal conductivity $(\kappa)$, and magnetostriction or thermal expansion $(\Delta L / L)$ as a function of magnetic field and/or temperature measurements are often used to confirm the existence of the zero-field energy gap and get a first glimpse at the shape, extension, and possible anisotropy of the phase diagram. NMR and $\mu \mathrm{SR}$ are probes of local magnetic fields, and can reveal important effects such as structural and magnetic modulations of the magnetic spins especially at fields higher than where neutron scattering is practical. Inelastic neutron scattering (INS) performed in singlecrystalline samples and electron spin resonance (ESR) are essential to directly determine the spin gap as well as the strength and sign of all relevant exchange interactions in the system. These tools will also verify the size of any uniaxial symmetry-breaking terms. Once this characterization has been done, the magnitude of the spin gap, exchange interactions, and symmetry-breaking terms can then be used to construct a minimal model which, in turn, is used to predict or replicate the $(T, H)$ phase diagram and physical properties. An important, although not very frequent, characterization tool is Young's modulus, to determine elastic properties and predict the effect of external pressure $(P)$. 


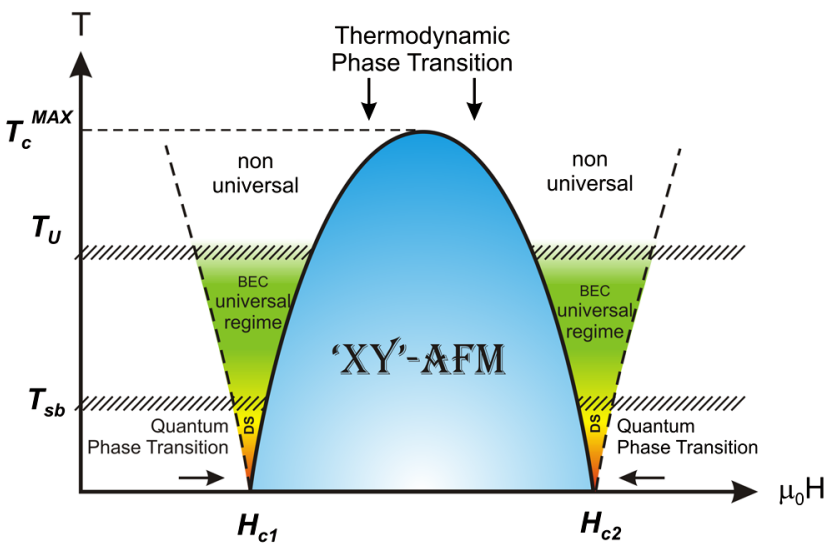

FIG. 10 (color online). Conceptual $(T, H)$ phase diagram for a quantum magnet with a field-induced QCP belonging to the BEC universality class. The region of magnetic ordering is delimited by the critical fields $H_{c 1}$ and $H_{c 2}$ and the maximum ordering temperature $T_{\mathrm{cmax}}$ in the $(T, H)$ plane. The energy scale $T_{s b}$ is the lower limit for observing a BEC QCP, and is given by uniaxialsymmetry-breaking terms below which discrete symmetry (DS) becomes important to the QCP. $T_{U}$ is the maximum temperature expected for universal properties that are derived from lowtemperature approximations to the boson distribution function, such as $T^{3 / 2}$ scaling of thermodynamic properties for a $d=3$ BEC QCP.

One can then circle back and test the predictions for a BEC QCP, especially the power-law scalings of different physical properties including the specific heat, magnetization, NMR relaxation rate, and critical fields at low temperatures. Some of these exponents are listed in Table III. Figure 10 is a typical phase diagram that shows the regions in $(T, H)$ space where the universal power-law scalings from Table III should occur. These regions are very close to $H_{c 1}$ and $H_{c 2}$ and they are bounded by $T_{s b}$ and $T_{U} . T_{s b}$ is the crossover temperature below which uniaxial-symmetry-breaking terms become significant and induce a crossover from the BEC-QCP scaling to a different universality class associated with the spontaneous breaking of a discrete symmetry. In particular, the asymptotic behavior of the QCP is Ising-like in dimension $D=d+1$ for compounds where the uniaxial symmetry axis for the spins acquires a twofold symmetry at low temperatures, and $X Y$-like in $D=d+1 \quad(d>1)$ when the uniaxial symmetry axis acquires a $p$-fold symmetry and $p \geq 4$ (Siegert and Everts, 1989; Miyashita, 1997; Pleimling, Neubert, and Siems, 1998). $T_{U}$ is the crossover temperature between universal and nonuniversal behavior at higher temperatures. The BEC description of the phase transition is still valid above $T_{U}$; however, the power laws are not observed because they are approximations for low $T$. Higher-order contributions of the expansion in the lattice gas parameter become important and the behavior of a given thermodynamic property depends on the microscopic details of each system. The exact value of $T_{U}$ depends on the property, e.g., magnetization, specific heat, or thermal expansion, that is being measured. Thus ideally the region between $T_{s b}$ and $T_{U}$ should extend over at least a decade of temperature $\left(T_{s b} \ll T_{U}\right)$ so the power laws can be accurately measured.
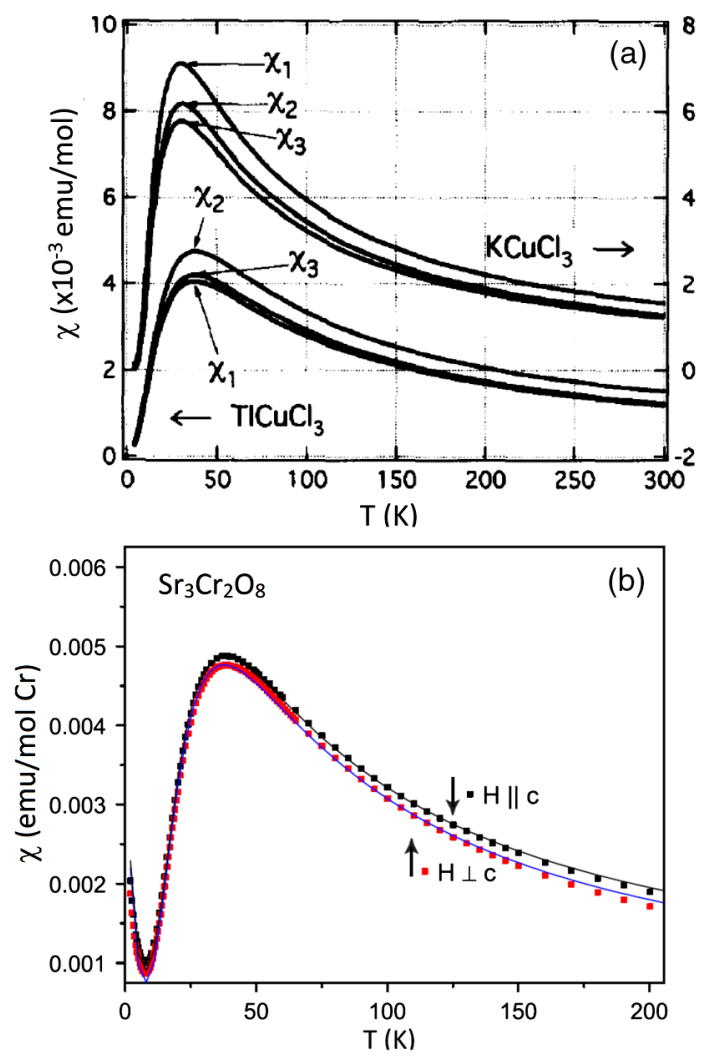

FIG. 11 (color online). (a) The temperature dependence of the magnetic susceptibilities of $\mathrm{KCuCl}_{3}$ and $\mathrm{TlCuCl}_{3}$ measured at $H=5 \mathrm{kOe} . \chi_{1}$ is the susceptibility for $H$ perpendicular to the cleavage plane, while $\chi_{2}$ and $\chi_{3}$ are the maximum and minimum susceptibilities when $H$ is applied in the cleavage plane, respectively. From Tanaka et al., 1997. (b) Magnetic susceptibility vs temperature $\chi(T)$ of $\mathrm{Sr}_{3} \mathrm{Cr}_{2} \mathrm{O}_{8}$ measured in a magnetic field of $10 \mathrm{kG}$ showing fits with Eq. (72) for $H \| c$ and $H \perp c$. The $g$ factors are fixed in the fits to the values obtained by ESR $g_{c}=$ 1.938 and $g_{a b}=1.950$. The fitting indicates $0.8 \%$ free $S=1 / 2$ moments, with the rest being dimerized, and the estimated exchange constants are $\Delta=J=61.5(3) \mathrm{K}$ (intradimer) and $J^{\prime}=$ 12(2) K (interdimer). From Aczel, Kohama, Marcenat et al., 2009.

The phase diagram in Fig. 10 also shows the region of ordering in the $(T, H)$ plane labeled $X Y$-AFM. In real magnets, this state is not a true $X Y$ AFM because the spins will be pinned along an easy axis in the $X Y$ plane. Even though uniaxial-symmetry-breaking terms that produce this pinning are very small and can be neglected at the BEC QCP, once the spins order, a phenomenon known as correlated pinning occurs. The small uniaxial-symmetrybreaking terms then act in the same way on every spin and the spins are rigidly coupled to each other in the ordered state. Therefore, the anisotropy energy is multiplied by the number of ordered spins and the spins cannot rotate freely in the $X Y$ plane.

\section{Magnetization}

Quantum magnets that exhibit BEC typically have a zerofield gap $\Delta$, which produces a prominent maximum in the magnetic susceptibility versus temperature near the gap energy (see Fig. 11). The location of the maximum is dictated 
by the crossover from an exponential increase for temperatures $k_{B} T \ll \Delta$ to a Curie-Weiss-type decrease for $k_{B} T \gg \Delta$. Strictly speaking, the magnetic susceptibility of such systems must be numerically computed taking into account the dispersion of magnetic excitations that is given by all relevant exchange interactions. However, the zeroth-order approximation is to assume that moments in different magnetic units do not interact with each other. A magnetic unit is a dimer, tetramer, or single spin depending on the compound. In this approach, we can discuss the magnetic energy levels of each magnetic unit cell that have energy $E_{j}$ and magnetization $m_{j}$ along the quantization axis defined by the orientation of the magnetic field. The magnetization per magnetic unit is then given by

$$
M=\frac{\sum_{j} g \mu_{B} m_{j} e^{-\left(E_{j}-g \mu_{B} m_{j} \mu_{0} H\right) / k_{B} T}}{\sum_{j} e^{-\left(E_{j}-g \mu_{B} m_{j} \mu_{0} H\right) / k_{B} T}} .
$$

The magnetic susceptibility is then calculated as $\chi=$ $d M / d H \simeq M / H$ for small $H$. In this way, for noninteracting spin $S=1 / 2$ dimers we obtain $\chi_{p}=2\left(\mu_{B}^{2} g^{2} / k_{B} T\right) /$ $\left(3+e^{\Delta / k_{B} T}\right)$, where $\Delta=J_{0}$ is the energy of the excited triplet state given by the intradimer exchange. The effect of exchange interactions between unit cells can be included at the mean-field level as an effective molecular field (Hara et al., 1971; Aczel, Kohama, Marcenat et al., 2009),

$$
\chi_{p}=\frac{2\left(g \mu_{B}\right)^{2}}{\left(3+e^{\Delta / k_{B} T}\right) k_{B} T+\frac{1}{2} \sum_{\nu \nu^{\prime} \eta} J_{\nu, \nu^{\prime}}^{\eta}} .
$$

The index $\eta$ runs over all dimers that have a finite exchange $J_{\nu \nu^{\prime}}^{\eta}$ with a given dimer, while $\nu$ and $\nu^{\prime}$ are internal indices for the two ions in each dimer (see Fig. 3).

Impurities are always present in a real compound, and these lead to an additional contribution $\chi_{\mathrm{imp}}=\nu_{\mathrm{imp}} \mu_{\mathrm{imp}}^{2} / k_{B} T$, where $\nu_{\text {imp }}$ is the concentration of magnetic impurities and $\mu_{\text {imp }}$ is their magnetic moment. Finally, there is a constant Van Vleck contribution $\chi_{0}$ that is present in any magnet and has to be added to reproduce the measured susceptibility. The final result is

$$
\chi=\chi_{p}+\frac{\nu_{\mathrm{imp}} \mu_{\mathrm{imp}}^{2}}{k_{B} T}+\chi_{0} .
$$

Ideally, data along different crystallographic directions are fitted, keeping all $J$ 's constant. This type of behavior is observed in quantum magnets with gapped zero-field ground states and was seen in most compounds in Table I. It is worth pointing out that, while useful to identify materials with potentially interesting zero-field gaps, these fits only serve as a rough estimate for the dominant interactions and $g$-factor anisotropy. A much better job can be done with inelastic neutron scattering and electron spin resonance techniques, which are discussed in upcoming sections.

Since an external magnetic field $H$ can be used to close the magnetic energy gap and induce magnetic order, an excellent way to characterize quantum magnets is the measurement of properties as a function of $H$. Magnetization $M(H)$ data allow us to estimate the strength of the effective AFM interdimer
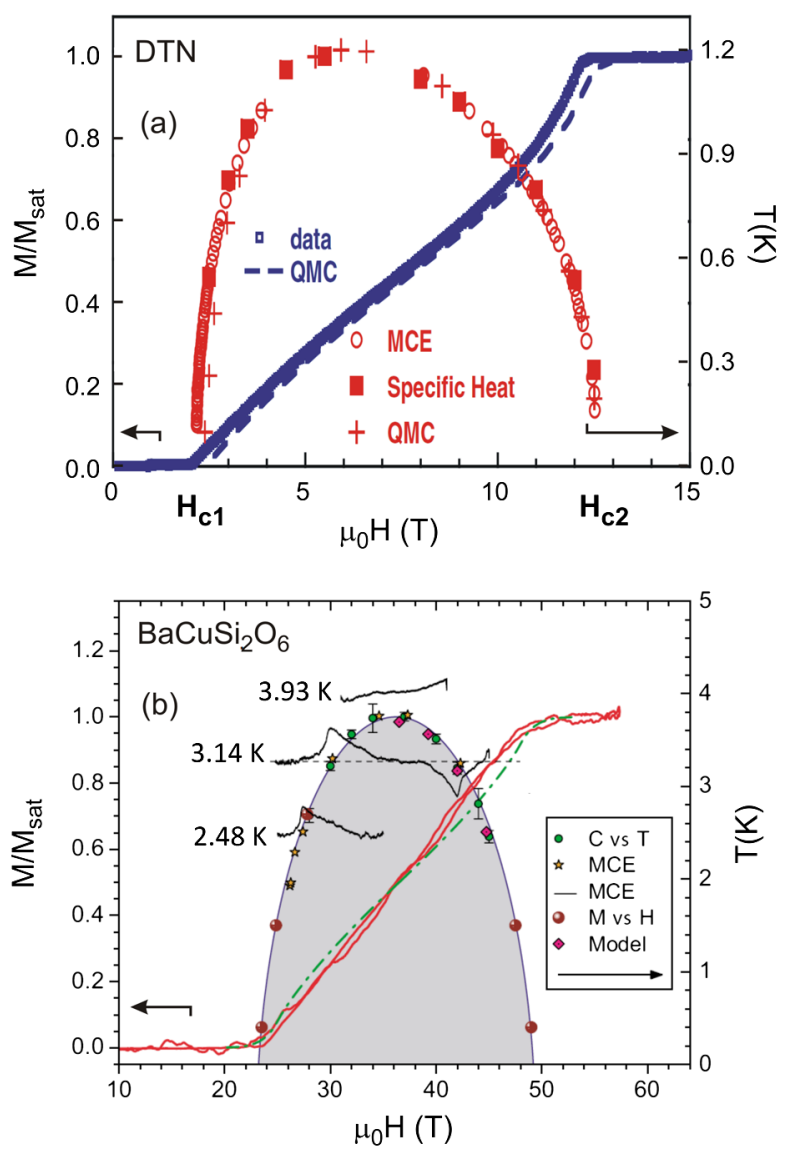

FIG. 12 (color online). Magnetization (left vertical axis) versus magnetic field $M(H)$ measured at low temperatures for (a) DTN $\left[\mathrm{NiCl}_{2}-4 \mathrm{SC}\left(\mathrm{NH}_{2}\right)_{2}\right]$ and (b) $\mathrm{BaCuSi}_{2} \mathrm{O}_{6}$. Critical fields $H_{c 1}$ and $H_{c 2}$ delimit the region of magnetic-field-induced long-range order. A temperature (right vertical axis) vs magnetic field $T_{c}(H)$ phase diagram is superimposed on the magnetization data for both materials. Adapted from Jaime et al., 2004, and Zvyagin et al., 2007.

exchange, because the width of the region between $H_{c 1}$ (onset of magnetization) and $H_{c 2}$ (saturation of magnetization) is related to the dispersion of the magnetic excitations of the quantum paramagnet. Figure 12 illustrates such measurement with early results in DTN and $\mathrm{BaCuSi}_{2} \mathrm{O}_{6}$. The critical magnetic fields are $\left(H_{c 1}=2 \mathrm{~T}, H_{c 2}=12.5 \mathrm{~T}\right)$ in the former and $\left(H_{c 1}=23.5 \mathrm{~T}, H_{c 2}=49 \mathrm{~T}\right)$ in the latter. $M(H)$ is zero between 0 and $H_{c 1}$, then increases roughly linearly between $H_{c 1}$ and $H_{c 2}$ as the spins cant in the direction of the applied magnetic field, and then saturates above $H_{c 2}$. This characteristic $M(H)$ behavior is another indicator of a potential BEC candidate. The $(T, H)$ phase diagram obtained from magnetization as well as specific heat $C$ and magnetocaloric effect delineates the region where magnetic order is observed. Qualitatively, a broad $H_{c 2}-H_{c 1}$ ordered region implies stronger AFM exchange interactions between magnetic units. We will see in Sec. III.B.5 that $H_{c 1} \rightarrow 0$ leads to enhanced zero-point fluctuations that modify the boson mass and create strong phase boundary asymmetry (particle-hole asymmetry) (Kohama et al., 2011).

The temperature dependence of the longitudinal magnetization $M_{z}(T)$ at constant magnetic field $H_{c 1}<H<H_{c 2}$, 
calculated by Nikuni et al. (2000) using a Hartree-Fock approximation for treating a gas of interacting bosons (Popov, 1987), is an indicator for a BEC QCP. Nikuni's results for $M_{z}(T)$ near the phase boundary are

$$
\begin{gathered}
\frac{n(T)}{n\left(T_{c}\right)}=\left(\frac{T}{T_{c}}\right)^{3 / 2}, \quad T \geq T_{c}, \\
\frac{n(T)}{n\left(T_{c}\right)}=2-\left(\frac{T}{T_{c}}\right)^{3 / 2}, \quad T<T_{c} .
\end{gathered}
$$

These expressions result in a minimum in $M(T)$ at $T=T_{c}$, and qualitatively explain the experimental results in several compounds including $\mathrm{TlCuCl}_{3}$ (Oosawa, Ishii, and Tanaka, 1999), $\mathrm{NiCl}_{2}-4 \mathrm{SC}\left(\mathrm{NH}_{2}\right)_{2}$ (Paduan-Filho, Gratens, and Oliveira, 2004b), and $\mathrm{Pb}_{2} \mathrm{~V}_{3} \mathrm{O}_{9}$ (Waki et al., 2004) (see Fig. 13).

\section{Specific heat}

The specific heat generally shows a sharp $\lambda$-like anomaly at the 3D ordering transition, which can be used to map the phase diagram. An example of specific heat measurements is shown in Fig. 14(a) for $\mathrm{TlCuCl}_{3}$ (Oosawa, Katori, and Tanaka, 2001). These measurements reveal a magnetic anomaly that grows and shifts in temperature as the magnetic field is increased above $H_{c 1}$. In Fig. 14(b) we show low-temperature specific heat measurements for $\mathrm{BaCuSi}_{2} \mathrm{O}_{6}$ covering a broad range of magnetic fields (Jaime et al., 2004). Due to the high critical magnetic fields in $\mathrm{BaCuSi}_{2} \mathrm{O}_{6}$, measurements were performed in a calorimeter made out of plastic materials (Jaime et al., 2000) using both a superconducting magnet and the $45 \mathrm{~T}$ hybrid magnet at the National High Magnetic Field Laboratory. In $\mathrm{BaCuSi}_{2} \mathrm{O}_{6}$, the $\lambda$-like anomalies in $C(T)$ are the same at low and high magnetic fields, i.e., there is a mirror symmetry about the field $\mu_{0} H=36 \pm 1 \mathrm{~T}$. This symmetry of the specific heat matches the mirror symmetry of the phase diagram. As explained in Sec. II.D.1, the mirror symmetry offers an experimental hint for an emergent particlehole symmetry in $\mathrm{BaCuSi}_{2} \mathrm{O}_{6}$ that results from the fact that higher-energy spin levels $\left|S^{z}=-1\right\rangle$ and $\left|S^{z}=0\right\rangle$ that break the mirror symmetry are so high above the ground state that they can be neglected.

Figure 15 shows the specific heat of $\mathrm{Cs}_{2} \mathrm{CuCl}_{4}$ (Radu et al., 2005). Unlike $\mathrm{TlCuCl}_{3}$, this material is gapless and ordered in zero field and the BEC QCP is reached where the gap opens at $H_{\text {sat. }}$. In addition to the $\lambda$-like peak due to the onset of long-range order, these data show a broad maximum at higher temperatures. This higher-temperature feature results from short-range intrachain spin correlations due to the quasi-1D nature of the compound. The overall shape of $C(T)$ above $T_{N}$ can be fitted by a numerical QMC simulation of a spin Hamiltonian determined from fits of INS data. In the gapped state for $H>H_{c}$ (not shown), the magnetic portion of the specific heat can be fitted by $C_{p} \sim e^{-\Delta / k_{B} T} / T$ at $T>0.4 \mathrm{~K}$ where the magnons are quasi-2D, and it can be fitted by $C_{p} \sim e^{-\Delta / k_{B} T} / \sqrt{(T)}$ below $0.15 \mathrm{~K}$ where the magnons becomes 3D. From these fits the value of the
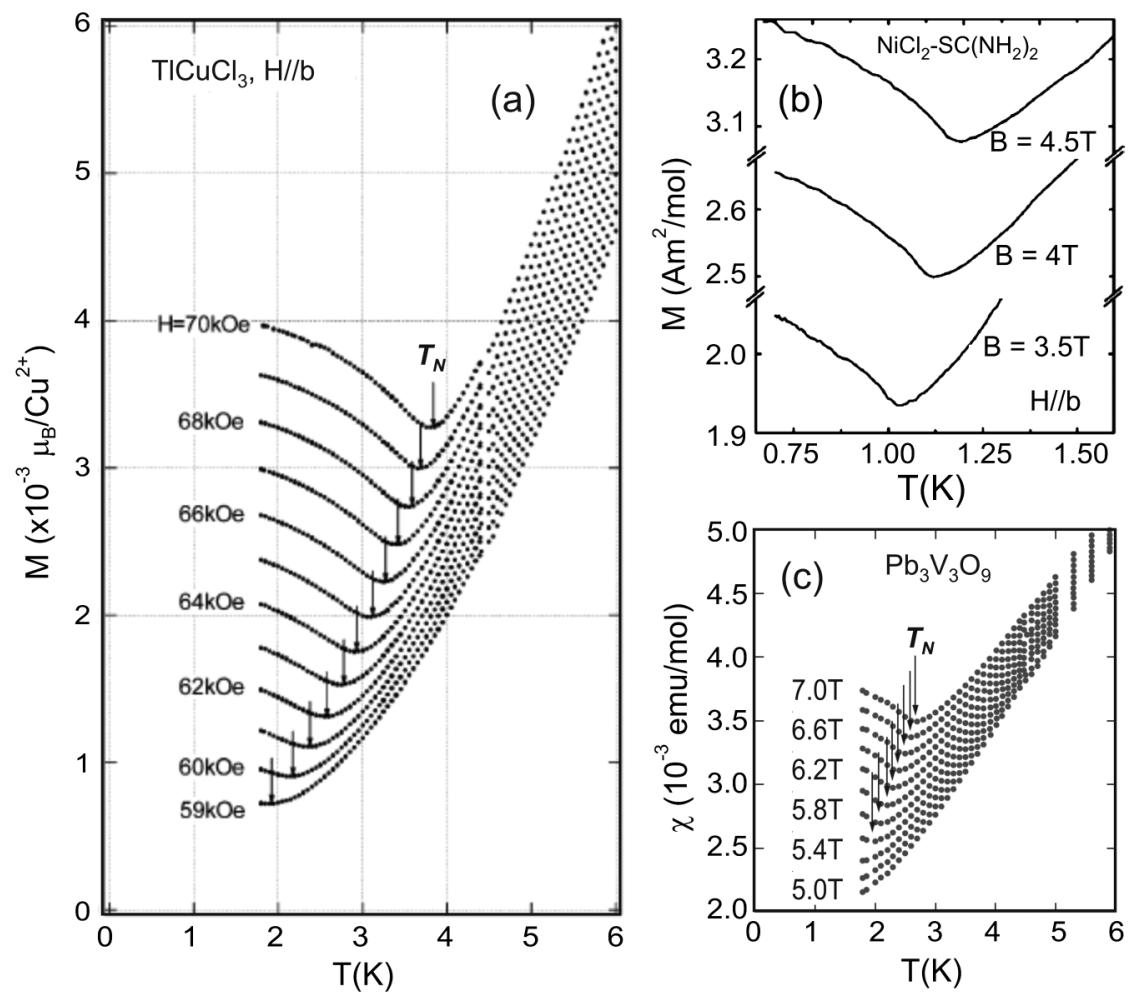

FIG. 13. Magnetization vs temperature $M(T)$ measured at constant magnetic fields for (a) $\mathrm{TlCuCl}_{3}$ (Oosawa, Ishii, and Tanaka, 1999), (b) DTN $\left[\mathrm{NiCl}_{2}-4 \mathrm{SC}\left(\mathrm{NH}_{2}\right)_{2}\right]$ (Paduan-Filho, Gratens, and Oliveira, 2004b), and (c) $\mathrm{Pb}_{2} \mathrm{~V}_{3} \mathrm{O}_{9}$ (Waki etal., 2004). A clear minimum in $M(T)$ is observed in all three compounds, as expected for a BEC. 

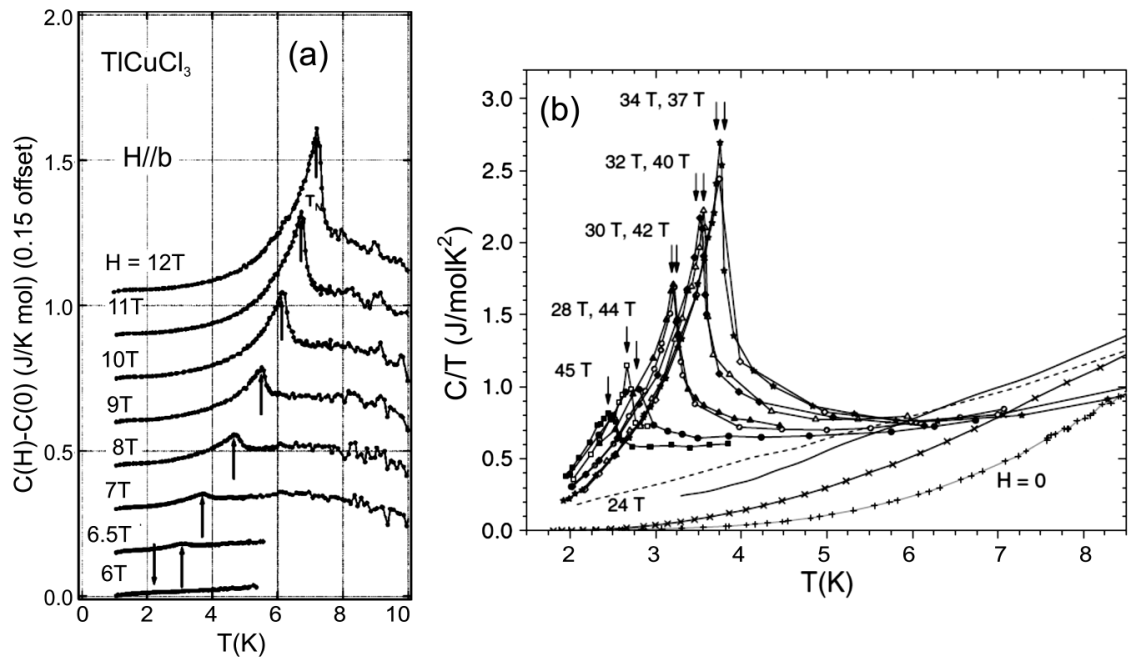

FIG. 14. (a) Magnetic contribution to the specific heat $C$ vs temperature $T$ measured in $\mathrm{TlCuCl}_{3}$. From Oosawa, Katori, and Tanaka, 2001. (b) Total $C(T) / T$ for $\mathrm{BaCuSi}_{2} \mathrm{O}_{6}$ for a number of magnetic fields $H<H_{c 2}$. These data show a remarkable symmetry for magnetic fields around $H\left(T_{c \max }\right)$ due to particle-hole symmetry. From Jaime et al., 2004.

gap can be extracted (Radu et al., 2005). With careful specific heat measurements approaching the critical field, it was shown that the gap closes at $H_{\text {sat }}$ to within the resolution of the measurements.
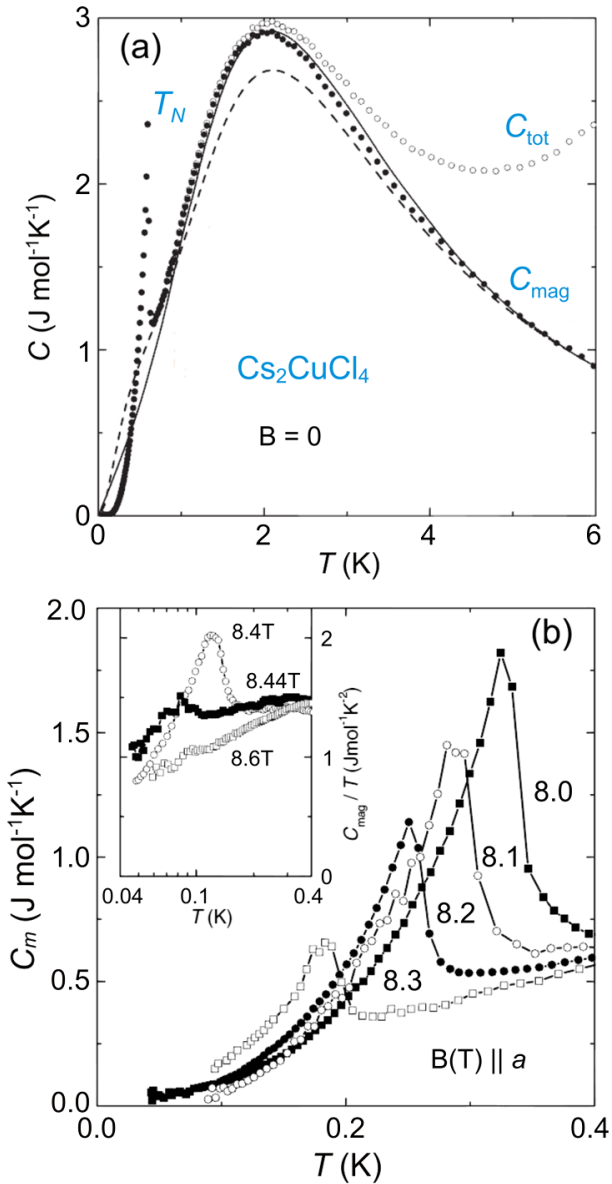

FIG. 15 (color online). (a) High-temperature specific heat vs temperature $C(T)$ measured in $\mathrm{Cs}_{2} \mathrm{CuCl}_{4}$ from which the spin energy gap is estimated. (b) Magnetic contribution to the specific heat measured for a number of applied magnetic fields showing a $\lambda$-like 3D ordering peak. From Radu et al., 2005.
In addition to $C(T), C(H)$ can also be directly compared to the results of QMC simulations of quantum magnets. One of the main advantages of this measurement is that the nonmagnetic contributions to $C(H)$ do not play an important role, and the effect of $H$ is directly observed (Kohama et al., 2011), as shown in Fig. 16 for DTN. In this compound the rising $\left|S^{z}=-1\right\rangle$ state is still low in energy near $H_{c 1}$ (see Fig. 7) and therefore breaks the mirror symmetry of the phase diagram, so the specific heat peak at the higher critical field $H_{c 2}$ is significantly larger than the peak at $H_{c 1}$. These data show broad shoulders in $C(T)$ below $H_{c 1}$ and above $H_{c 2}$ due to the opening of a spin gap in these paramagnetic regions.

\section{Magnetocaloric effect}

The magnetocaloric effect (MCE) is a sometimes underappreciated tool for extracting information about quantum magnets. It can be used to determine the phase diagram, often

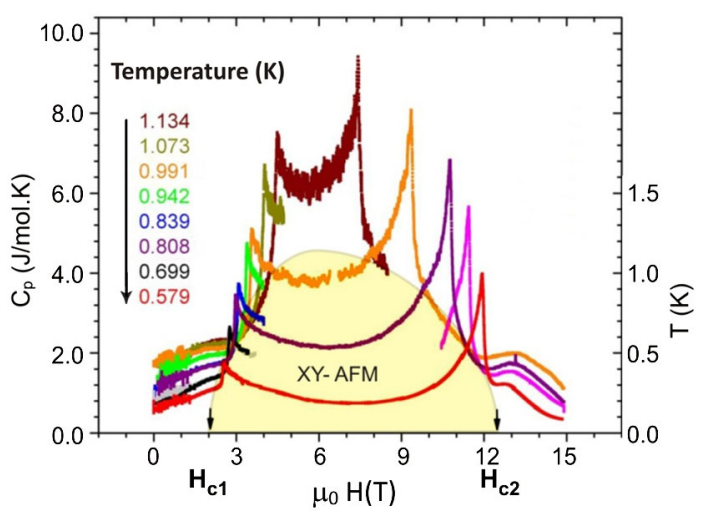

FIG. 16 (color online). Specific heat $C$ vs magnetic field $H$ for DTN $\left[\mathrm{NiCl}_{2}-4 \mathrm{SC}\left(\mathrm{NH}_{2}\right)_{2}\right]$ obtained using an ac technique. $C(H)$ measured at several temperatures show sharp anomalies at the critical magnetic fields $H_{c 1}(T)$ and $H_{c 2}(T)$. The contribution from the sample holder was not subtracted. The light shaded region is the $X Y$ AFM phase in the $(H, T)$ phase diagram of DTN From Kohama et al., 2011. 
much more quickly than the specific heat. MCE data can also be used to extract the entropy versus temperature and magnetic field, and thereby predict the magnetization and the rest of the thermodynamic parameters of a material. It provides useful information about the nature of phase transitions, e.g., whether they are first or second order, and it can be used to estimate the entropy involved in any phase transition or crossover. When a sample is subjected to a changing magnetic field $H$, its entropy changes as the spin configuration evolves. The entropy increases when the spin gap becomes smaller than $k_{B} T$ while it decreases above the ordering transition $\left[H>H_{c 1}(T)\right]$. Thus, these two regimes can be readily distinguished. Under adiabatic or semiadiabatic conditions, the changes in spin entropy are balanced by opposite changes in the lattice entropy, which in turn affect the temperature. For example, the MCE is central to the principle of magnetic cooling and adiabatic demagnetization refrigerators. A typical MCE measurement measures the temperature of the lattice via a thermometer that is in intimate thermal contact with the sample, as the magnetic field is varied. The sample and thermometer should be somewhat isolated from the external environment, and depending on the degree of this isolation, the MCE can be measured in different limits, e.g., adiabatic, quasiadiabatic or equilibrium. Understanding these limits is critical to interpreting the data.

If the sample is linked to an infinite thermal bath by a thermal conductivity $\kappa$ that has no thermal mass of its own, then the time constant for the temperature of the sample to relax to the bath is $\tau_{1}=C_{\text {sample }} / \kappa$. If we define $\tau_{\exp }$ as the time scale under which the magnetic field is swept, e.g., the time needed to traverse a phase transition, adiabatic conditions correspond to $\tau_{1} \gg \tau_{\text {exp }}$, and semiadiabiatic conditions correspond to $\tau_{1} \sim \tau_{\exp }$. The equilibrium limit corresponds to $\tau_{1} \ll \tau_{\exp }$, but $\kappa$ is not infinitely large, so temperature changes will still be observed. This is a useful limit as we will see in the coming discussion. The relaxation time between the sample and the thermometer, $\tau_{2}$, is an additional parameter to consider. We will assume in this section that $\tau_{2} \ll \tau_{1}, \tau_{\text {exp. }}$. The analysis would become more complicated for $\tau_{2} \sim \tau_{1}$ or $\tau_{2} \sim \tau_{\text {exp }}$ because we need to take into account the delayed response of the thermometer to the sample's heat changes.

Figure 17(a) illustrates the different experimental regimes in a MCE experiment. Typical curves are shown for a fieldinduced phase transition from disorder to order, under adiabatic, semiadiabatic, and equilibrium conditions, as well as the fully thermalized condition $(\kappa \rightarrow \infty)$, where $T$ does not change with $H$.

Typical $T(H)$ curves are shown in Fig. 17(b) for a quantum paramagnet that exhibits a field-induced ordering transition. The main effect on the paramagnetic region comes from the linear reduction of the spin gap as a function of the applied field. Consequently, one would expect to observe the first anomaly in the magnetocaloric effect when the spin gap becomes of the order of $k_{B} T$. We note that this anomaly should be distinguishable from the second one that appears at the ordering transition as long as $k_{B} T$ is a significant fraction of $k_{B} T_{c \max }$. In the following we describe how these different anomalies become manifest in the adiabatic, semiadiabatic, and equilibrium regimes.

Under adiabatic conditions, and assuming $\tau_{2}$ is negligibly small, the Langevin expression for $T(H)$ is

$$
\left(\frac{\partial T}{\partial H}\right)_{S}=-\frac{T}{C_{H}}\left(\frac{\partial M}{\partial T}\right)_{H}
$$
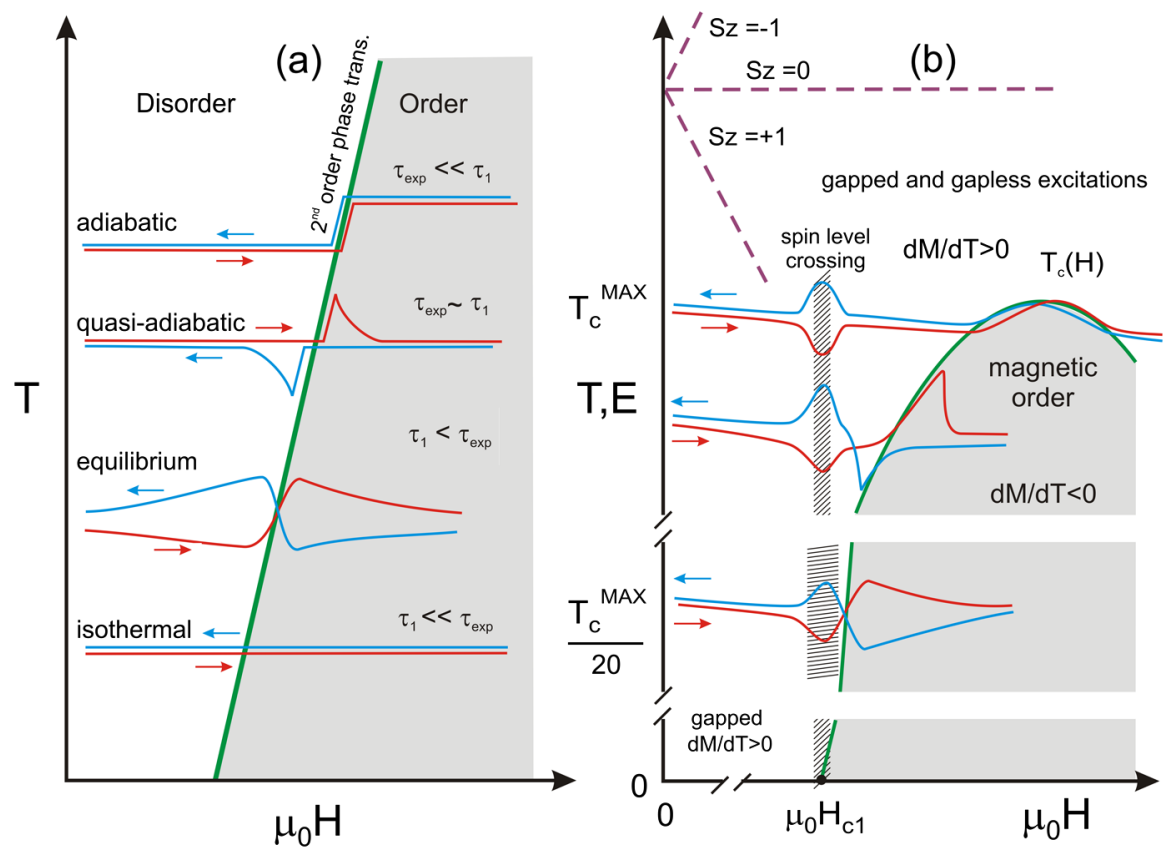

FIG. 17 (color online). (a) Typical $T(H)$ traces at a field-induced disorder-order transition. From top to bottom, curves are shown for the adiabatic $\left(\tau_{1} \gg \tau_{\text {exp }}\right)$, semiadiabatic $\left(\tau_{1} \sim \tau_{\exp }\right)$, quasi-isothermal $\left(\tau_{1} \ll \tau_{\text {exp }}\right)$, and completely thermalized $(\kappa \rightarrow \infty)$, where $\tau_{1}=C_{\text {sample }} / \kappa$. Note that the size of the jumps are not to scale-the jump in the adiabatic case would be largest. (b) $T(H)$ traces at a field-induced ordering transition in a system that also has spin levels that cross, e.g., the quantum magnets with BEC QCPs. 
where $T$ is the temperature, $H$ is the magnetic field, $S$ is the entropy, $M$ is the magnetization, and $C_{H}$ is the sample specific heat at constant magnetic field. From Eq. (75) and the magnetization data shown in Fig. 13 one can extract the $T(H)$ curve that is expected for $\mathrm{TlCuCl}_{3}$ in the adiabatic limit.

The $T(H)$ curve should exhibit an upward jump at the fieldinduced ordering transition. If all phase transitions are of second order, $T(H)$ will jump down at the same transition when the field is swept down. In fact the entire $T(H)$ curve will be reversible, e.g., identical on magnetic field upsweeps and downsweeps. Any entropy gained in sweeping the field up will be lost on sweeping the field down. Irreversibility in this adabiatic limit indicates a first-order phase transition with latent heat, or irreversible heat releases from, e.g., sudden structural changes. In the adiabatic limit [see Fig. 17(a)], $T(H)$ can directly be used to extract the entropy and the magnetization from Eq. (75). Figure 17(b) shows that $H_{c 1}$ corresponds to a minimum in $T(H)$ for an ordering transition under adiabatic conditions. This is consistent with the fact that $\delta M / \delta T$ also shows a minimum at $H_{c 1}$ (see Fig. 13), and indicates that entropy is maximized at the phase transition. We note that entropy is reduced deep inside the ordered $T \ll T_{c}$ and paramagnetic phases $k_{B} T \ll \Delta(H)$. Representative data in the adiabatic regime are shown in Fig. 18 for DTN.

Under semiadiabatic conditions, the sample relaxes back to the bath temperature with a relaxation time $\tau_{1}$. This can be a difficult limit in which to interpret the data because the temperature changes due to relaxation can occur on the same time scales as the temperature changes due to the changing
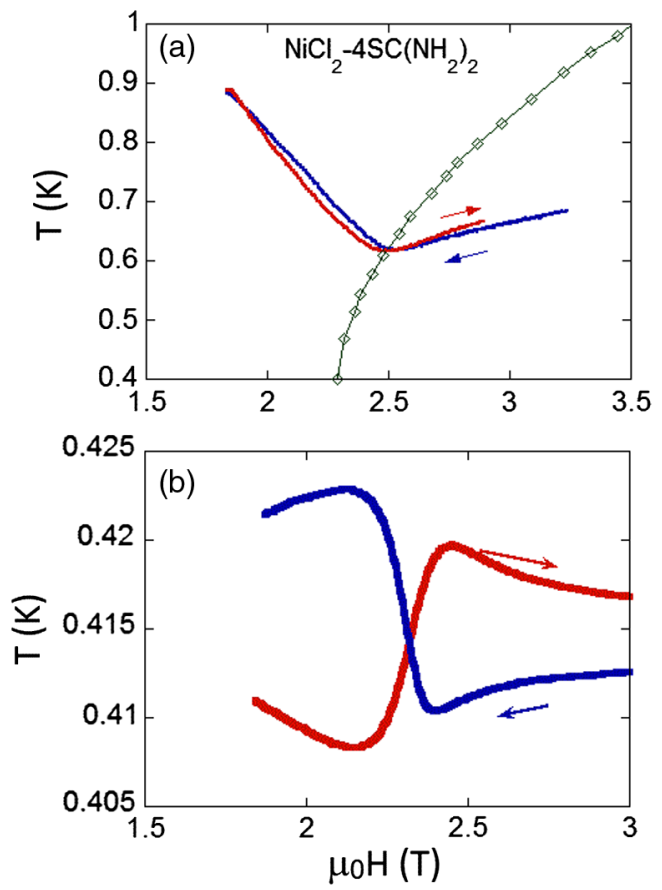

FIG. 18 (color online). (a) Magnetocaloric effect for DTN $\left[\mathrm{NiCl}_{2}-4 \mathrm{SC}\left(\mathrm{NH}_{2}\right)_{2}\right]$ in the adiabatic limit, near $H_{c 1}$ (unpublished). (b) Magnetocaloric effect in the equilibrium limit near $H_{c 1}$ (in which the data were incorrectly interpreted as showing irreversible behavior). The different limits are achieved by varying the sample size and the magnetic field sweep rate from Zapf et al. (2006). magnetic field. The parameters $\kappa$ (thermal conductance of the thermal link) and $\tau_{1}$ (characteristic time constant) need to be well-characterized in order to extract quantitative information about the entropy. $T(H)$ is governed by (Kohama et al., 2010)

$$
\kappa \Delta T=\kappa \tau_{1}\left(\frac{\partial T}{\partial H}\right)\left(\frac{d H}{d t}\right)+T\left(\frac{\partial S}{\partial H}\right)_{T}\left(\frac{d H}{d t}\right) .
$$

Note that Eq. (76) assumes that the thermal link $\kappa$ has no thermal mass. The curves in Figs. 17(a) and 17(b) show that in this limit $T(H)$ shows signs of exponential behavior governed by $\tau_{1} . T(H)$ is not reversible in this regime, even though there are no first-order phase transitions. Data in this regime are shown in Fig. 19 for $\mathrm{BaMn}_{2} \mathrm{O}_{8}$ (Samulon et al., 2009). $\mathrm{BaCuSi}_{2} \mathrm{O}_{6}$ (Jaime et al., 2004) is another example for which MCE data have been published in the semiadiabatic limit. There are some indications that the $T(H)$ curve wants to follow the $T_{c}(H)$ critical field line in certain regimes (see Figs. 19 and 20). One possibility is a stabilization at the critical field. The sample heats up as the magnetic field is increased and the sample transitions into the ordered phase, which could drive it back across the phase boundary into the disordered region. At that point the sample tries to cool again, which brings it back toward the phase boundary. This effect might be realized when the lattice specific heat is small compared to the spin specific heat, so that heating just inside the ordered phase is large. Furthermore, $T(H)$ given in Eq. (76) contains two terms under semiadiabatic conditions. These terms can add just inside the phase boundary so as to amplify the heating. Whether this phase-boundary-hugging effect in $T(H)$, hinted at in the semiadiabatic data of Fig. 19, occurs deserves further study.

Finally, we consider the equilibrium regime $\tau_{1} \ll \tau_{\text {exp }}$. Note that this limit does not mean that the sample's temperature remains constant as the field is swept. A MCE effect will occur for any noninfinite $\kappa$ and can once again be used to

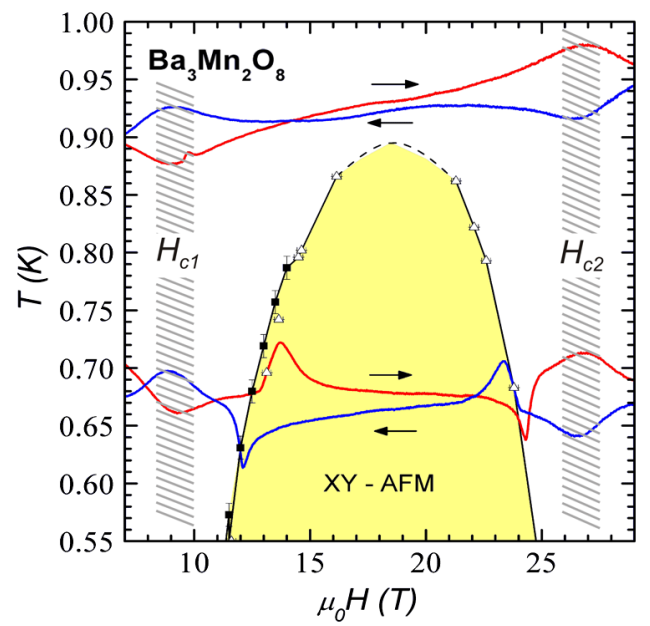

FIG. 19 (color online). Magnetocaloric effect and specific heat vs temperature were used to build the $(H, T)$ phase diagram for $\mathrm{Ba}_{3} \mathrm{Mn}_{2} \mathrm{O}_{8}$, shown here for $H \| c$. The magnetocaloric effect was measured under semiadiabatic conditions and shows an anomaly near the spin level crossing, and another set of anomalies at $H_{c 1}$ and $H_{c 2}$. Adapted from Samulon et al., 2008. 

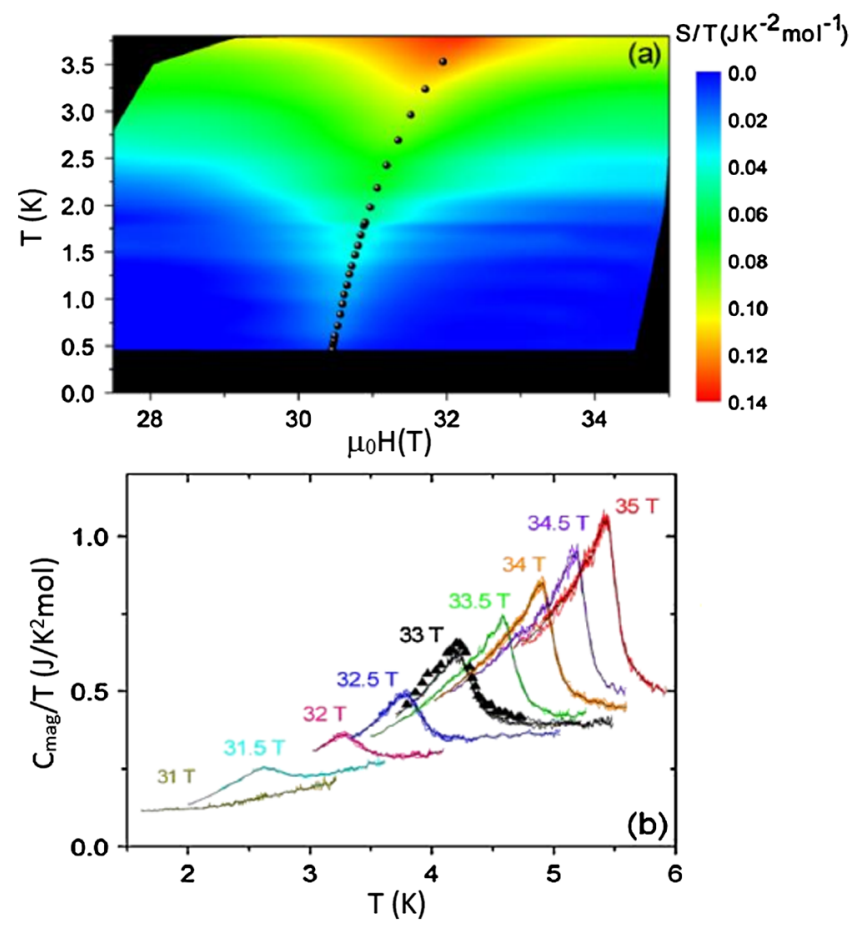

FIG. 20 (color online). (a) Phase diagram of $\mathrm{Sr}_{3} \mathrm{Cr}_{2} \mathrm{O}_{8}$ near $H_{c 1}$, with the entropy extracted from Eq. (75) shown as a contour plot. (b) Phase diagram and magnetocaloric effect for $\mathrm{Sr}_{3} \mathrm{Cr}_{2} \mathrm{O}_{8}$ in the equilibrium limit for $T<4 \mathrm{~K}$ and the nearly adiabatic limit for $T>4 \mathrm{~K}$ from Aczel, Kohama, Marcenat et al. (2009).

extract the entropy. The equation governing this regime is $P=\kappa \Delta T$, where $P$ is the heat rate associated with the changing entropy, which in turn escapes over the thermal link $\kappa$ at a rate determined by the temperature difference $\Delta T$ between the sample and the bath. We call it the "equilibrium limit" because the sample temperature is never governed by $\tau_{1}$ or $\tau_{2}$-there is never a time when the sample temperature is relaxing back to the bath at an exponential rate. Substituting $P=d Q / d t=(d Q / d H)(d H / d t)=C(d T / d H)(d H / d t)$ and employing Eq. (75) we obtain

$$
T(H)=-\frac{T}{\kappa} \frac{d H}{d t}\left(\frac{\partial M}{\partial T}\right)_{H}
$$

Once again, the magnetization, entropy, and the rest of the thermodynamic quantities can be extracted from $T(H)$. Typical curves are shown in Figs. 17(a) and 17(b). Note that $\partial M / \partial T$ is now proportional to $T(H)$, whereas in the adiabatic case $\partial M / \partial T$ was proportional to $\partial T / \partial H$. Thus, in the equilibrium limit the $T(H)$ curves look like derivatives with respect to $H$ of the curves in the adiabatic case. Note also that the $T(H)$ traces on upsweep and downsweep are inverses of each other because $d H / d t$ that appears in Eq. (77) changes sign on the downsweep. By contrast, $T(H)$ was reversible without any inversion in the adiabatic case.

Measurements in the equilibrium or near-equilibrium limit were performed, for example, in the compounds DTN (Zapf et al., 2006), (HPIP) $)_{2}-\mathrm{CuBr}_{4}$ (Thielemann, Rüegg, Kiefer et al., 2009), $\mathrm{BaCr}_{2} \mathrm{O}_{8}$ (Aczel, Kohama, Jaime et al., 2009), and $\mathrm{Sr}_{3} \mathrm{Cr}_{2} \mathrm{O}_{8}$ (for $T<4 \mathrm{~K}$ ) (Aczel, Kohama, Marcenat et al., 2009). This equilibrium limit requires very slow sweep rates such as are found in superconducting or dc resistive magnets. MCE data for DTN taken in a superconducting magnet in the equilibrium limit are shown in Fig. 18(b). MCE data in the equilibrium limit for $\mathrm{Sr}_{3} \mathrm{Cr}_{2} \mathrm{O}_{8}$ in a dc resisitive magnet are shown in Fig. 20 for $T>3 \mathrm{~K}$. Here the entropy is extracted from $T(H)$ and shown in a contour plot.

Thus the magnetocaloric effect can map the phase diagram, determine the order of the phase transitions, and extract the entropy and thereby the rest of the thermodynamic properties of the sample. Additional theoretical and experimental discussions of the MCE at general phase transitions and QCPs can be found in Garst and Rosch (2005), Silhanek et al.(2006), and Wolf et al. (2010).

\section{Thermal conductivity}

As discussed in Sec. II.B, quantum magnets should not exhibit the transport properties of superfluid ${ }^{4} \mathrm{He}$ due to the absence of an actual superfluid response for times longer than $\tau_{m}$. The first experimental exploration of thermal conductivity $(\kappa)$ by magnons (Kudo et al., 2004) revealed some enhancement at the transition to the ordered state of $\mathrm{TlCuCl}_{3}$ both in the $T$ dependence and in the $H$ dependence. These findings were later echoed by a qualitative discussion of data in $\mathrm{Pb}_{2} \mathrm{~V}_{3} \mathrm{O}_{9}$ (Sato et al., 2010). If the temperature is significantly smaller than the spin gap of the quantum paramagnet, the number of magnetic excitations as a function of magnetic field has maxima at the critical fields $H_{c 1}$ and $H_{c 2}$. This is so because the dispersion relation is gapless and quadratic $(z=2)$ at the BEC QCPs, gapless and linear inside the ordered region, and gapped on the paramagnetic sides. In other words, the low-energy density of states has maxima at the critical fields. Therefore, the observation of maxima in the low-temperature $\kappa(H)$ near the critical fields indicates that magnons give the predominant contribution to $\kappa$, and the maxima occur because the number of carriers (magnons) is maximized around those fields. We note, on the other hand, that the phonon contribution should have minima around the critical fields because the scattering of phonons by magnetic excitations should be proportional to the density of magnetic excitations. Therefore, the thermal conductivity is dominated by magnons if the $\kappa(H)$ curve measured at low temperature has maxima around the critical fields, while it is dominated by phonons if it has minima. Measurements of $\kappa(H)$ in the $S=1$ DTN (Ke et al., 2011; Kohama et al., 2011) show a strong enhancement at the critical fields $H_{c 1}(T)$ and $H_{c 2}(T)$, consistent with thermal transport dominated by magnons, whereas most other systems in this paper show a local minimum in $\kappa$ consistent with thermal transport dominated by phonons.

The dominant scattering mechanism for the magnon contribution to the thermal conductivity is unclear without a quantitative analysis. However, it is clear that scattering with impurities should dominate at low enough temperatures because the density of magnons and phonons goes to zero for $T \rightarrow 0$. Detailed measurements of $\kappa(T)$ in DTN and $\mathrm{Ba}_{3} \mathrm{Mn}_{2} \mathrm{O}_{8}$ (Sun et al., 2009; Ke et al., 2011; Kohama et al., 2011) have shown 

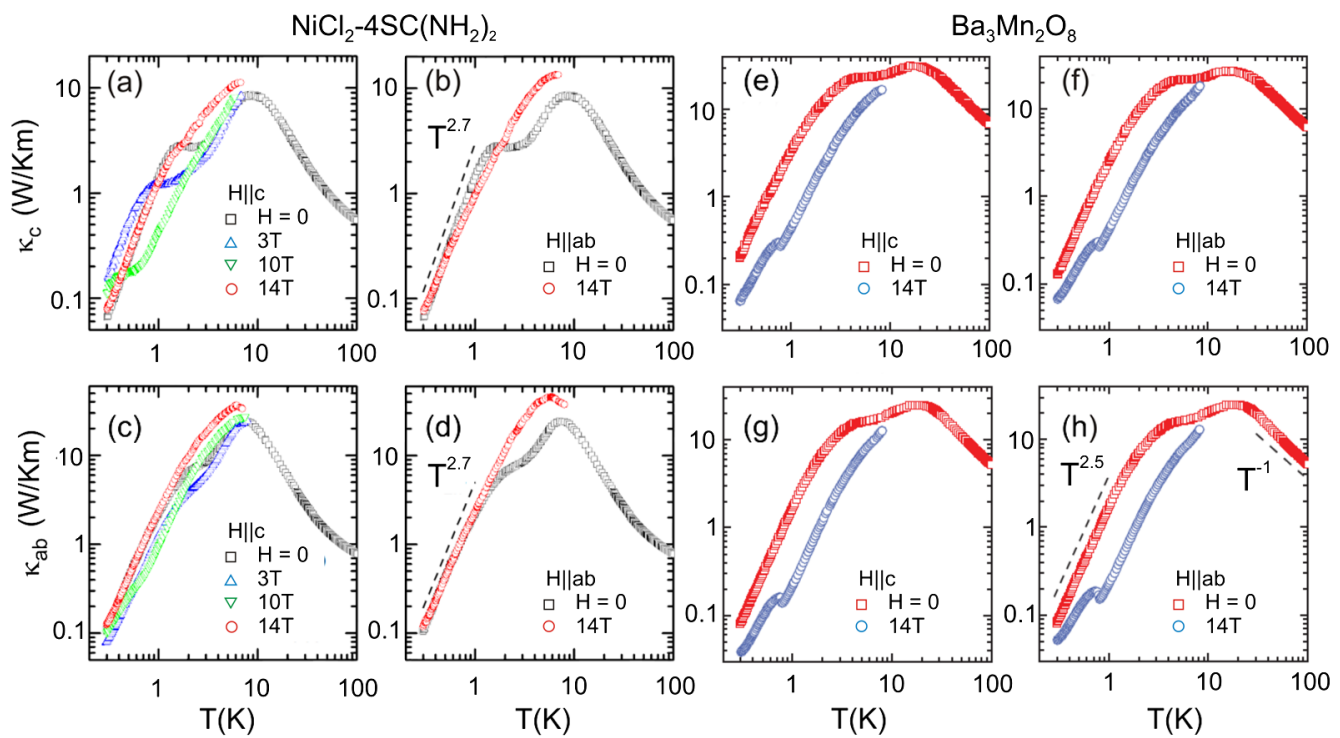

FIG. 21 (color online). Thermal conductivity $\kappa$ vs temperature $T$ along the crystallographic $a$ and $c$ axes measured in DTN $\left[\mathrm{NiCl}_{2}-4 \mathrm{SC}\left(\mathrm{NH}_{2}\right)_{2}\right]$ and $\mathrm{Ba}_{3} \mathrm{Mn}_{2} \mathrm{O}_{8}$ for different magnetic field values (Ke et al., 2011; Kohama et al., 2011).

intriguing behaviors. The thermal conductivity $\kappa(T)$ measured for field orientation parallel and perpendicular to the crystallographic $c$ axis (see Fig. 21) show similar characteristics for both systems, i.e., a broad maximum at $T \sim 8-18 \mathrm{~K}$ likely due to phonon heat conduction and a shoulder at lower temperatures that moves toward $T=0$ as the applied magnetic field increases. This shoulder was interpreted as originating from resonant magnetic scattering. Below this shoulder, $\kappa$ obeys a power-law temperature dependence with an exponent between 2.5 and 2.7, somewhat smaller than the cubic dependence expected for phonons in the boundary scattering limit. All BEC-like systems show evidence of the $X Y$-AFM transition for magnetic fields $H_{c 1}<H<H_{c 2}$ and $\kappa(T)$ is enhanced below the ordering temperature $T_{c}(H)$ (see Fig. 21).

\section{Evidence of quantum fluctuations: Asymmetry of the phase diagram}

An interesting aspect of magnetic realizations of bosonic gases is the possibility of probing the effect of quantum fluctuations on the boson mass. The boson mass is a property of the single-particle dispersion relation, $1 / \mathrm{m}^{*}=$ $\partial^{2} \epsilon /\left.\partial k^{2}\right|_{k=Q}$. In particular, we have seen already that the dispersion relation is gapped and quadratic in the paramagnetic regions $H<H_{c 1}$ and $H>H_{c 2}$ (e.g., the bosons have a finite mass outside of the ordered phase) and becomes linear and gapless (e.g., massless) inside the ordered phase. [A Goldstone mode appears inside the ordered phase due to the spontaneous breaking of the continuous $U(1)$ symmetry.] The bosonic masses in the two paramagnetic regions do not need to be the same because the zero-point fluctuations below $H_{c 1}$ can be much stronger than the corresponding fluctuations above $H_{c 2}$. To understand this statement we need to notice that the fully polarized product state that appears above $H_{c 2}$ is the exact ground state because it is the only state which has the maximum value of the magnetization $M_{z}$ along the field direction. The conservation of $M_{z}$ implies that this state cannot be mixed with any other state. Being a product state, we can say that the paramagnetic ground state above $H_{c 2}$ has no intersite zero-point fluctuations. In contrast, the total magnetization is $M^{z}=0$ below $H_{c 1}$ and the exact ground state is a linear combination of an infinite number of product states. For instance, if we are considering a dimerized quantum magnet, the direct product of singlet states on each dimer is mixed by the Hamiltonian with states in which a pair of singlets connected by interdimer exchange become a pair of triplets with net magnetization equal to zero. This means that a triplet (boson) created below $H_{c 1}$ moves in a background of quantum fluctuations. These zero-point AFM fluctuations have the effect of reducing the bosonic mass. Indeed, we know that this mass should vanish when the zero-point AFM fluctuations diverge, i.e., when the zero-field spin gap is closed by the application of pressure. We have seen already that the corresponding pressure-induced QCP has dynamical exponent $z=1$, implying that the bosons become massless because the single-particle dispersion relation is linear in momentum. Therefore, the bare mass $m$ for $H>H_{c 2}$ should be much larger than the effective mass $m^{*}$ for $H<H_{c 1}$, whenever the ratio $H_{c 1} /\left(H_{c 2}-H_{c 1}\right)$ is much smaller than 1 .

This effect was studied in $\mathrm{Ba}_{3} \mathrm{Mn}_{2} \mathrm{O}_{8}$, which has two ordered phases due to triplet and quintuplet condensation (Samulon et al., 2009). The $T-H$ phase diagram in Fig. 8(d) shows slight but noticeable asymmetries in the dome shape of the ordered phases. The specific heat versus temperature and magnetic field $C(T, H)$ shown in Fig. 22 illustrates the asymmetry between $H_{c 3}$ and $H_{c 4}$ in this material, with larger peaks and thus higher effective bosonic masses near $H_{c 4}$.

The asymmetry is even more pronounced in DTN because of the small ratio $H_{c 1} /\left(H_{c 2}-H_{c 1}\right) \sim 1 / 5$ (Kohama et al., 2011). The mass tensors for $H<H_{c 1}$ and $H>H_{c 2}$ are obtained from the single-boson dispersions in each paramagnetic state,

$$
\frac{1}{m_{\nu \nu}^{*}}=\left.\frac{\partial^{2} \tilde{\omega}_{\mathbf{k}}^{<}}{\partial k_{\nu}^{2}}\right|_{\mathbf{k}=\mathbf{Q}}, \quad \frac{1}{m_{\nu \nu}}=\left.\frac{\partial^{2} \tilde{\omega}_{\mathbf{k}}^{>}}{\partial k_{\nu}^{2}}\right|_{\mathbf{k}=\mathbf{Q}} .
$$




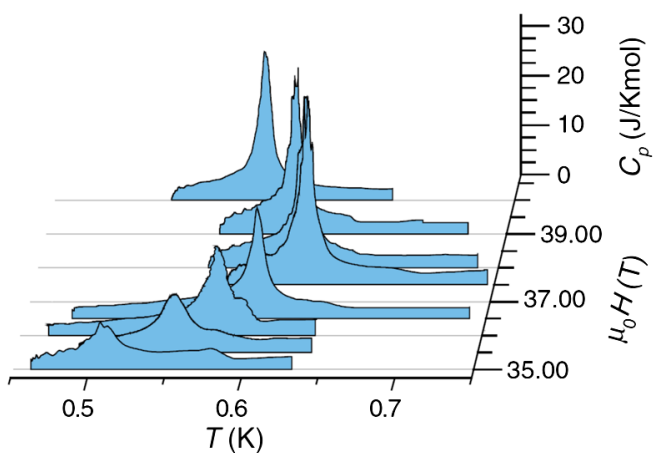

FIG. 22 (color online). Specific heat $C_{p}$ vs temperature $T$ and magnetic field $H$ of $\mathrm{Ba}_{3} \mathrm{Mn}_{2} \mathrm{O}_{8}$ showing the ordered transitions between $H_{c 3}$ and $H_{c 4}$ in this material. From Samulon et al., 2009.

where the $<$ sign holds for $H<H_{c 1}$ and the $>$ sign holds for $H>H_{c 2}$. The dispersion relation $\tilde{\omega}_{\mathbf{k}}^{<}$was derived in Sec. II.D.2 [see Eq. (60)], while the exact dispersion relation above $H>H_{c 2}$ is

$$
\tilde{\omega}_{\mathbf{k}}^{>}=\epsilon_{\mathbf{k}}-\epsilon_{\mathbf{Q}}+h-h_{c 2} .
$$

Then, the mass renormalization factor is given by

$$
\frac{m_{\nu \nu}}{m_{\nu \nu}^{*}}=s^{2} \frac{\mu}{\omega_{\mathbf{Q}}^{0}} \approx \frac{H_{c 2}}{4 H_{c 1}}\left(1+\sqrt{1+\frac{8 H_{c 1}^{2}}{H_{c 2}^{2}}}\right) .
$$

For the parameters of the Hamiltonian from Zvyagin et al. (2007), we obtain $m_{\nu \nu} / m_{\nu \nu}^{*} \simeq 3.2$. The large asymmetry between $m$ and $m^{*}$ is reflected in different physical properties at $H_{c 1}$ and $H_{c 2}$ as shown in Figs. 23 and 16, such as the shape of the phase boundary, the specific heat (Zapf et al., 2006), magnetization (Paduan-Filho, Gratens, and Oliveira, 2004b), magnetostriction (Zapf et al., 2008; Weickert et al., 2012), ESR (Zvyagin et al., 2007), and sound velocity (Chiatti et al.,

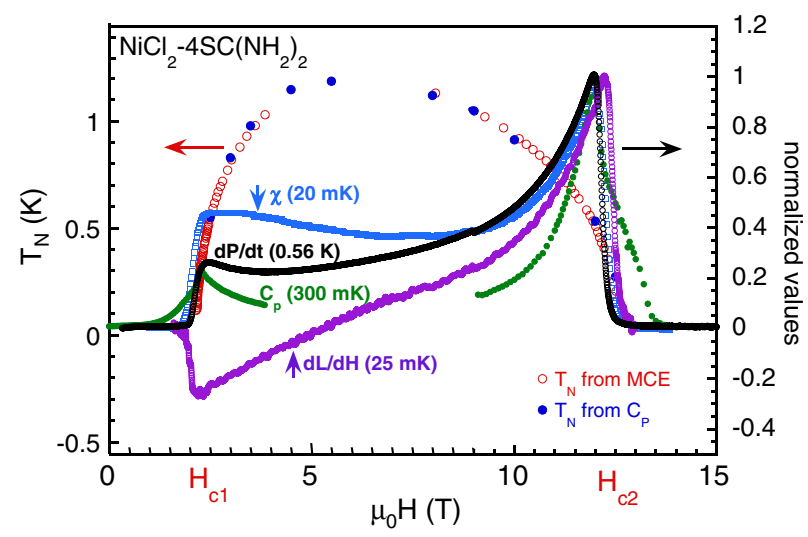

FIG. 23 (color online). Asymmetry between physical properties of DTN $\left[\mathrm{NiCl}_{2}-4 \mathrm{SC}\left(\mathrm{NH}_{2}\right)_{2}\right]$ at $H_{c 1}$ and $H_{c 2}$. Here $\chi$ is the magnetic susceptibility, $C_{p}$ is the specific heat, $d L / d H$ is the magnetostriction, $d P / d t$ is the magnetoelectric current due to changes in the electric polarization of the sample, and $T_{N}$ is the Néel temperature determined from the magnetocaloric effect (open symbols) and the specific heat (solid symbols) (Zapf et al., 2006; 2011; Yu et al. 2008; Kohama et al., 2011).

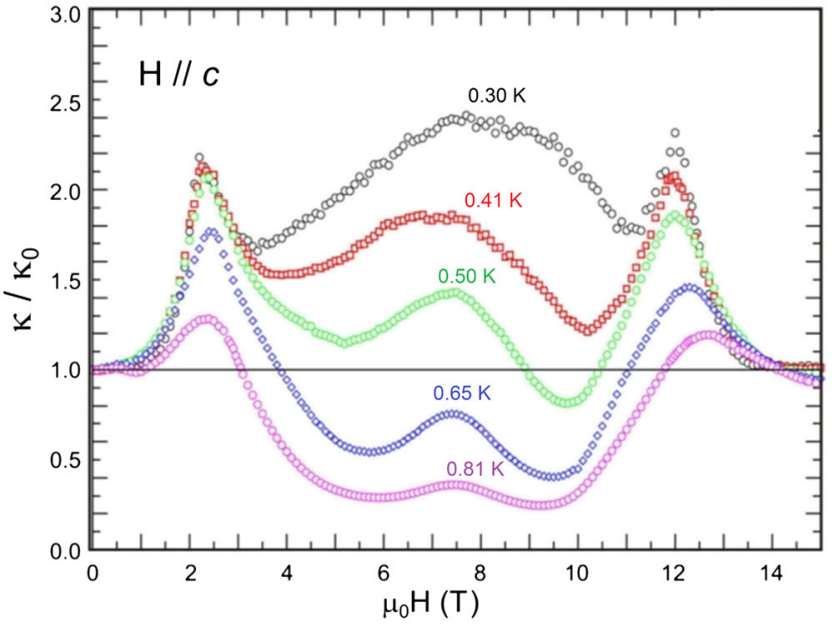

FIG. 24 (color online). Thermal conductivity $\kappa$, along the $c$ axis as function of $\mathbf{H} \| c$ for DTN $\left[\mathrm{NiCl}_{2}-4 \mathrm{SC}\left(\mathrm{NH}_{2}\right)_{2}\right] . \kappa$ is measured at constant temperatures between $T=0.3$ and $0.81 \mathrm{~K}$, showing a clear enhancement at the critical fields $H_{c 1}$ and $H_{c 2}$ From Kohama et al., 2011.

2009; Zherlitsyn et al., 2009). Kohama et al. (2011) showed that the large asymmetry between the peaks in the lowtemperature specific heat $C(H)$ in the vicinity of $H_{c 1}$ and $H_{c 2}$ is closely described by analytical and QMC calculations. The mass renormalization also explains similar asymmetries observed in other properties of DTN. In a remarkable contrast to these properties, peaks in the low-temperature thermal conductivity $\kappa$ near $H_{c 1}$ and $H_{c 2}$ do not show any substantial asymmetry (see Fig. 24). Kohama et al. (2011) provided an explanation to this dichotomy by demonstrating that the leading boson-impurity scattering amplitude is also renormalized by quantum fluctuations, effectively canceling the mass renormalization effect in $\kappa$.

\section{Magnetic effects on the crystal lattice}

Important physical properties related to the spin-chargelattice coupling, such as sound velocity, magnetostriction, and electric polarization, were studied in several BEC quantum magnets. We note magnetostructural coupling is universal in magnetically ordered systems and does not automatically create first-order behavior or create significant terms in the Hamiltonian that would violate the conditions for BEC. Johannsen et al.(2005) analyzed the magnetoelastic coupling of $\mathrm{TlCuCl}_{3}$ based on high-resolution measurements of the thermal expansion and the magnetostriction. By combining linear thermal expansion measurements along different crystallographic axes in constant magnetic fields with published specific heat data and Ehrenfest relations, they found that the phase boundaries $T_{c}(p)$ and $H_{c 1}(p)$ between the low-field paramagnetic phase and the ordered phase are extremely sensitive to pressure and change by about $185 \%$ GPa depending on the direction of uniaxial pressure. This drastic effect can be traced back to the pressure dependence of the intradimer exchange, whereas changes of the interdimer couplings play a minor role. Substitution of $\mathrm{Tl}$ for K, however, causes the opposite effect, i.e., mostly a reduction in the dispersion. 

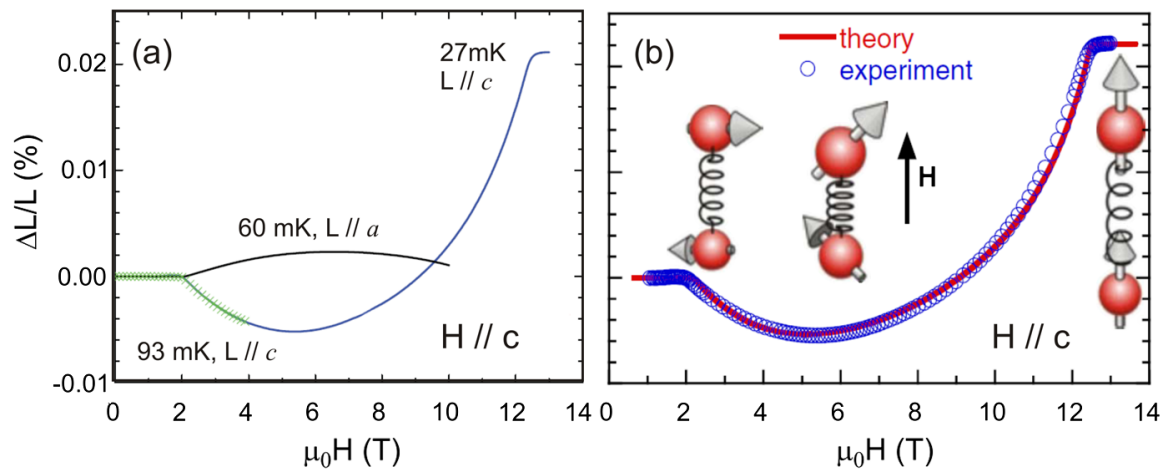

FIG. 25 (color online). (a) Magnetostriction $\Delta L / L$ measured along the $c$ and $a$ crystallographic axes vs magnetic field $\mathbf{H}$ applied along the $c$ axis. (b) Experimental (open circles) and numerically simulated (line) magnetostriction $\Delta L / L$ of $\mathrm{DTN}\left[\mathrm{NiCl}_{2}-4 \mathrm{SC}\left(\mathrm{NH}_{2}\right)_{2}\right]$ along the $c$ axis vs magnetic field (Zapf et al., 2008; Weickert et al., 2011).

Zapf et al. (2008) and Weickert et al. (2012) investigated the magnetostriction of DTN and found a lattice response to the field-induced $X Y$ AFM phase between the critical fields $H_{c 1}$ and $H_{c 2}$ (see Fig. 25). The longitudinal magnetostriction $\Delta L / L_{c}$ measured along the crystallographic $c$ axis shows a gradual non-symmetry-breaking shrinking above $H_{c 1}$ followed by a minimum and an expansion as the field approaches $H_{c 2}$ (Zapf et al., 2008). The volume stops changing for external magnetic fields above $H_{c 2}$. This behavior has been interpreted as a result of a strong $\mathrm{Ni}-\mathrm{Ni}$ distance dependence in $J_{c}$. Since the AFM exchange constant increases as the Ni-Ni distance shrinks, the AFM component of the spin ordering induces magnetostriction, while the field-induced FM component produces magneto expansion. Indeed, the AFMstaggered component dominates at low fields $H \gtrsim H_{c 1}$, because it increases like $\sqrt{H-H_{c 1}}(\beta=1 / 2$ is the meanfield exponent expected for a BEC QCP in dimension $D=3+2$ ), while the uniform component increases linearly in $H-H_{c 1}$ (see Sec. II.C). This observation explains why the lattice shrinks for low fields and expands for high fields. These measurements are extremely well reproduced by the results of quantum Monte Carlo simulations of the Hamiltonian given in Eq. (52), by assuming that the main contribution to the magnetostriction arises from the change in the exchange constant $J_{c}$ as a function of the lattice parameter along the $c$ axis. Under this assumption, the relative change of the sample length along the $c$ axis $\Delta L_{c} / L_{c}$ is proportional to the correlation function between two neighboring spins along the same axis: $\left\langle\mathbf{S}_{\mathbf{r}} \cdot \mathbf{S}_{\mathbf{r}+\mathbf{e}_{\mathbf{c}}}\right\rangle$ (Zapf et al., 2008). From the proportionality constant between the measured $\Delta L_{c}(H) / L_{c}(H)$ curve and the simulated correlation function $\left\langle\mathbf{S}_{\mathbf{r}} \cdot \mathbf{S}_{\mathbf{r}+\mathbf{e}_{\mathbf{c}}}\right\rangle(H)$, Zapf et al. (2008) obtained the dependence of $J_{c}$ on inter- $\mathrm{Ni}^{2+}$ distance $d J_{c} / d c=2.5 \mathrm{~K} / \AA$ by also using the value of the Young's modulus $E_{33}=7.5 \pm 0.7 \mathrm{GPa}$ that was measured for this purpose. Similar measurements and analysis were performed for (HPIP) $)_{2}-\mathrm{CuBr}_{4}$ (Anfuso et al., 2008), although in the 1D Luttinger liquid regime, and excellent agreement was found with spin-spin correlation function calculations for a two-leg spin ladder model.

Ultrasonic measurements provide a powerful experimental technique to study various phase transitions and critical phenomena. It is also a well-established tool for the investigation of low-dimensional spin systems. Spin-lattice interactions are responsible for the attenuation of acoustic waves and influence the sound velocity in magnetic crystals. These interactions are connected either with a strain modulation of the exchange interactions or with a magnetostrictive coupling of a single-ion type. Chiatti et al. performed measurements of the relative change of the sound velocity and attenuation in $\mathrm{NiCl}_{2}-4 \mathrm{SC}\left(\mathrm{NH}_{2}\right)_{2}$ using a phase-sensitive detection technique, based on a standard pulse-echo method (Chiatti et al., 2008, 2009; Zherlitsyn et al., 2009). The acoustic $c 33$ mode manifests a pronounced softening accompanied by energy dissipation of the sound wave in the vicinity of the quantum critical points. The acoustic anomalies are traced up to $T>T_{N}$, where the thermodynamic properties are well described by fermionic magnetic excitations because the interchain coupling becomes less relevant (as discussed in Sec. II.A, the magnetic Hamiltonian can be mapped into a gas of interacting fermions for one-dimensional systems). On the other hand, the 3D ordering present in the AFM phase implies
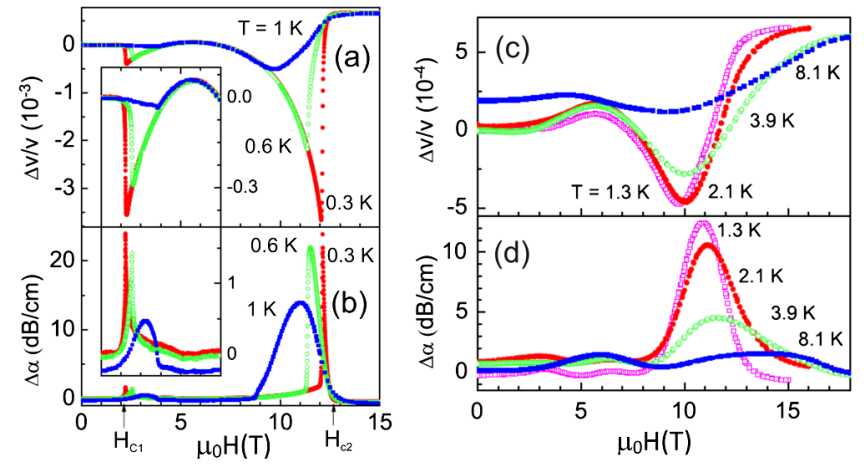

FIG. 26 (color online). (a) Field dependence of the relative change of the sound velocity and (b) the sound attenuation of the acoustic $c 33$ mode in DTN $\left[\mathrm{NiCl}_{2}-4 \mathrm{SC}\left(\mathrm{NH}_{2}\right)_{2}\right]$ at temperatures below the maximum Néel temperature $T_{N}$. The magnetic field was applied along the (001) axis. The ultrasonic frequency was $78 \mathrm{MHz}$. The insets show the sound velocity and attenuation in the vicinity of $H_{c}$ in an enlarged scale. (c) Field dependence of the relative change of the sound velocity and (d) the sound attenuation of the acoustic $c 33$ mode in DTN for temperatures above the maximum $T_{N}$ Froom Chiatti et al., 2008. 
that the system has to be treated as a gas of interacting bosons for $T<T_{N}$ in order to reproduce the experimental results (the fermionic approach is no longer a good approximation because the interchain coupling becomes relevant at low temperatures). Indeed, the experimental results indicates a crossover from a high-temperature regime [Fig. 26(d), $8.1 \mathrm{~K}$ data] that is well described by a $1 \mathrm{D}$ fermionic model to a lowtemperature 3D regime [Fig. 26(d), 1.3 K data], where the actual bosonic character of the magnetic excitations becomes evident (Chiatti et al., 2008).

\section{Critical exponents for the thermal properties near the BEC QCP}

As discussed earlier in Sec. II.C, a field-induced BEC QCP leads to universal properties in the proximity of the critical fields $H_{c 1}(T=0)$ and $H_{c 2}(T=0)$. A number of experiments have attempted to check the validity of Eq. (24) for $H_{c 1}(T)$ and $H_{c 2}(T)$ in BEC quantum magnets. In addition, thermodynamic quantities should follow power-law temperature dependencies at $H=H_{c 1}$ and $H=H_{c 2}$. Table III shows the expected exponents for BEC and Ising-like QCPs (Zapf et al., 2011). It is important to note that $d=3$ is the upper critical dimension for the Ising-like QCP $(D=d+z=4)$ because the the dynamical exponent is $z=1$. Therefore, one should expect further logarithmic corrections to the power-law behaviors listed in Table III (Weickert et al., 2012).

A common test for the BEC universality class involves measurements of the ordering temperature as a function of the temperature-dependent critical field $T_{c}(H)$. A fit with Eq. (24) then provides a way to test the prediction in the first line of Table III. The problem, however, is that the range of temperatures over which data are available is often too small to accurately determine the exponent of the power law. Ideally, at least an order of magnitude should be available down to as low temperatures as possible. Furthermore, the powerlaw predictions are valid only in the window $T_{s b}<T<T_{U}$ (see Fig. 10). Thus, different values of $\phi$ can be determined by fitting over different temperature windows. Literally any value of the exponent $\phi$ can be obtained with an insufficient data set and a simplistic approach to fitting the power law.

Several groups have used the windowing technique to determine $\phi$ in $\mathrm{BaCuSi}_{2} \mathrm{O}_{6}, \mathrm{DTN}, \mathrm{TlCuCl}_{3}, \mathrm{KCuCl}_{3}$, and $\mathrm{CsCu}_{2} \mathrm{Cl}_{4}$ (Nohadani et al., 2004; Sebastian et al., 2005; Radu et al., 2006; Sebastian, Harrison 2006; Zapf et al., 2006; Tanaka et al., 2007; Yamada et al., 2008). Here the data are fitted over many different windows of temperature and then the fitting parameters are extrapolated toward $T=0$. In addition, an attempt is made to determine $H_{c}(0)$ and $\phi$ separately. First $H_{c}(0)$ is extracted from fits by setting a fixed value of $\phi$ and then repeating this for different fixed values of $\phi$. In experiments, the obtained $H_{c}(0)$ extrapolated toward $T \rightarrow 0$ is independent of the choice of $\phi$. This universal $H_{c}(0)$ value is then used to fit $\phi$, and once again the values of $\phi$ are extrapolated toward fitting windows with $T \rightarrow 0$.

Figure 27 shows the windowing approach applied to $\mathrm{BaCuSi}_{2} \mathrm{O}_{6}$. Based on the emergent particle-hole symmetry that should be approximately correct for this material (the interdimer exchange interactions are ten times smaller than the intradimer exchange), the empirical equation

$$
t=g\left(h^{2}\right)[(1-h)(1+h)]^{\phi} \equiv g\left(h^{2}\right)\left(1-h^{2}\right)^{\phi}
$$

was proposed to fit the experimental phase boundary in a window of increasing size $t_{w}$, where $t=T_{N} / T_{N \max }$ and
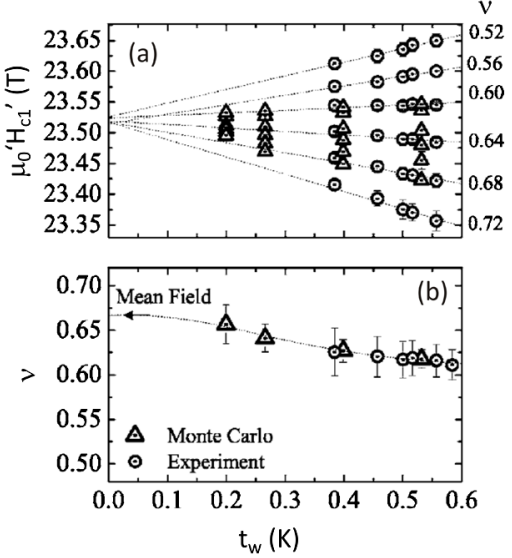
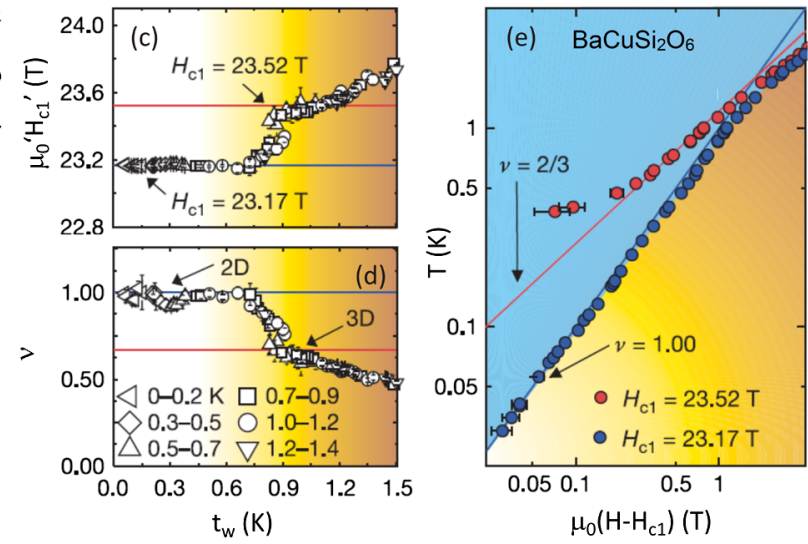

FIG. 27 (color online). Windowing analysis of the critical field $H_{c}(T)$ in $\mathrm{BaCuSi}_{2} \mathrm{O}_{6}$. (a) Circles represent estimates of $H_{c 1}$ obtained from fitting experimental points on the phase boundary of $\mathrm{BaCuSi}_{2} \mathrm{O}_{6}$ in a window of increasing size $t_{w}=T / T_{c \max }$, for different fixed values of $\nu=1 / \phi . t_{w}$ labels the highest reduced temperature of the fit window. The dotted lines show the linear convergence of $H_{c 1}$ values at $t_{w}=0$. Triangles represent estimates of $H_{c 1}$ similarly obtained from quantum Monte Carlo simulations for corresponding fixed values of $\nu$, and similar convergence is observed. (b) Circles represent estimates of $\nu$ from fitting experimental points on the phase boundary in a window of increasing size $t_{w}$, with $H_{c 1}=23.52 \mathrm{~T}$ determined from (a). The error bars are due to experimental uncertainty in determining values of $H_{c}$. Triangles represent estimates of $\nu$ from a similar fit to QMC simulation data. The dotted line is a guide to the eye, illustrating the approach of the QMC data to the mean-field value as $t_{w} \rightarrow 0$. (c) The same as (a) with temperatures now reaching $30 \mathrm{mK}$ in a dilution refrigerator. A rapid change is found in the estimate for $H_{c 1}$ for $t_{w}<0.6$. (d) The same as (b), but again to $30 \mathrm{mK}$. The change in $H_{c 1}$ accordingly affects the zero-temperature limit for $\nu$, indicating a crossover from 3D to 2D universality as the temperature is dropped. (e) $T$ vs $H-H_{c 1}$ phase boundary plotted for the two different values of $H_{c 1}$ in (c) showing $\phi=1$ $\left(H_{c 1}=23.17 \mathrm{~T}\right)$ and $\phi=3 / 2\left(H_{c 1}=23.52 \mathrm{~T}\right)$ behaviors (Sebastian et al., 2005; Sebastian, Harrison et al., 2006). 
$h=\left(H_{\max }-H\right) /\left(H_{\max }-H_{c 1}\right)$. Here $g\left(h^{2}\right)$ is assumed to be constant to a first approximation for different fixed values of $\phi$. It is empirically observed from linear extrapolation to $t_{w}=0$ that estimates of $H_{c 1}$ become less dependent on $\phi$ near the QCP and converge to a single value irrespective of the value of $\phi$ [Fig. 27(a)]. $H_{c 1} \simeq 23.52 \pm 0.03 \mathrm{~T}$ is estimated from this $H_{c 1}$ convergence. This value is then used to estimate the critical exponent $\phi$ by fitting Eq. (81) in the narrowest temperature range near the QCP that has a statistically significant number of data points. Figure 27(b) shows the variation in $\phi$ with the size of the temperature window. For temperature windows below $0.61 \mathrm{~K}$, a value of $1 / \phi=0.63 \pm 0.03$, or $\phi=1.59 \pm 0.08$ can be extrapolated, which is just about consistent with the theoretical meanfield prediction $\phi=3 / 2$. A similar analysis was also implemented for DTN (Zapf et al., 2006), $\mathrm{TlCuCl}_{3}$ (Tanaka et al., 2007), and $\mathrm{CsCu}_{2} \mathrm{Cl}_{4}$ (Radu et al., 2006), as well as for the phase diagram predicted by $\mathrm{QMC}$ calculations for $\mathrm{TlCuCl}_{3}$ and $\mathrm{KCuCl}_{3}$ (Nohadani et al., 2004).

Shortly after the first critical exponent study in $\mathrm{BaCuSi}_{2} \mathrm{O}_{6}$, Sebastian et al. (2005) extended their measurements to dilution-refrigeration temperatures (Sebastian, Harrison et al., 2006). They found an unexpected crossover from the threedimensional $\phi=3 / 2$ to the two-dimensional value $\phi=1$. Initially this dimensional crossover was attributed to the effects of 2D interlayer frustration. However, further experiments found a ferromagnetic non-frustrated interlayer exchange interaction as well as a modulation of the intradimer exchange constant along the crystallographic $c$ axis with uneven density of boson condensation that could account for the dimensional reduction as is explained in Sec. II.C (Sebastian, Tanedo et al., 2006; Batista et al., 2007; Rösch and Vojta, 2007; Schmalian and Batista, 2008; Laflorencie and Mila, 2009; Mazurenko et al., 2014). The exponent for DTN was also subsequently measured down to $1 \mathrm{mK}$ $\left(T / T_{\max }=1 / 1000\right)$ immersed in ${ }^{3} \mathrm{He}$ in an adiabatic

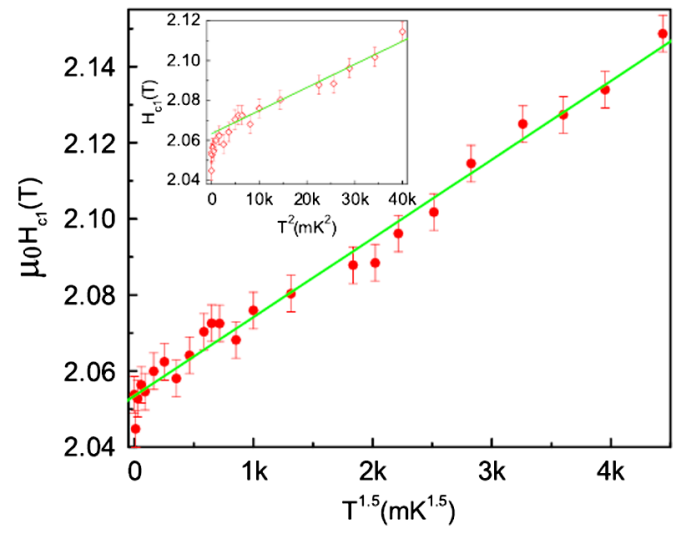

FIG. 28 (color online). Critical field $H_{c 1}$ vs $T^{1.5}$ for DTN $\left[\mathrm{NiCl}_{2}-4 \mathrm{SC}\left(\mathrm{NH}_{2}\right)_{2}\right]$ down to $1 \mathrm{mK}$, determined from ac susceptibility data. The observed linear behavior of this function is consistent with the expected BEC power-law exponent $\phi=3 / 2$. Deviations at low temperatures are eventually expected due to uniaxial-symmetry-breaking terms. The inset shows the curve $H_{c 1}\left(T^{2}\right)$ showing that the exponent $\phi=2$ expected for an Ising QCP in dimension $3+1$ is inconsistent with the experimental data From Yin et al., 2008. demagnetization fridge by using ac susceptibility at the high $B / T$ facility of the National High Magnetic Field Laboratory (Yin et al., 2008). The value of $\phi=3 / 2$ was confirmed by direct fits without windowing analysis over the temperature range 1-200 mK. These data are shown in Fig. 28.

Another important way of testing the universal properties of quantum magnets is to measure the temperature dependence of thermodynamic properties in close proximity to the critical magnetic fields. These quantities are expected to follow the simple power-law dependences shown in Table III. However, the high critical magnetic fields of many BEC compounds renders these experiments difficult. The magnetization is expected to show $T^{3 / 2}$ behavior up to higher temperatures than other thermodynamic properties. For DTN the magnetization is shown in Fig. 29(a) for temperatures up to $80 \%$ of $T_{c \max }$. Further measurements of magnetization, specific heat, and thermal expansion carried out down to $0.1 T_{c \max }=0.1 \mathrm{~K}$ in DTN were extensively analyzed and contrasted against both QMC and analytical results shown in Figs. 30 and 31 (Weickert et al., 2012). The measured properties do approach the theoretical expectations as the temperature is reduced, although the limited thermal conductivity of the sample below $0.1 \mathrm{~K}$ prevents reaching very low temperatures in some experimental setups in vacuum. The experimental results can be compared to theoretical predictions for temperatures higher than $T_{U}$ with the help of materials-dependent QMC calculations.
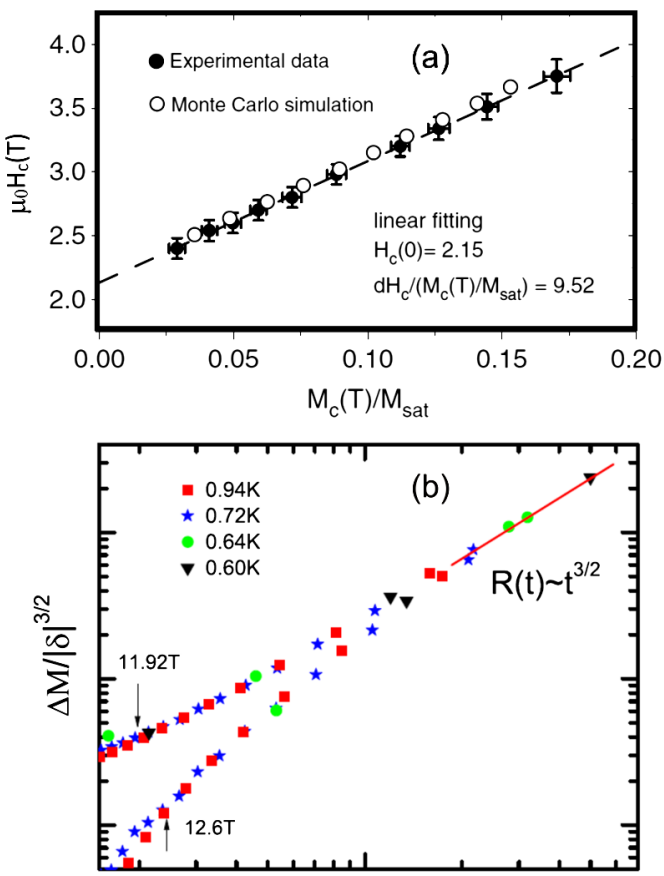

$\mathrm{T} /|\delta|$

FIG. 29 (color online). (a) Critical field $H_{c}(T)$ vs magnetization at the critical field $M_{c}(T) / M_{\text {sat }}$ for DTN $\left[\mathrm{NiCl}_{2}-4 \mathrm{SC}\left(\mathrm{NH}_{2}\right)_{2}\right]$. (b) Scaled magnetization vs temperature below and above the upper critical field showing the approach toward the $T^{3 / 2}$ dependence expected for a BEC QCP in spatial dimension $d=3$ (Reyes, Paduan-Filho, and Continentino, 2008; PaduanFilho et al., 2009). 

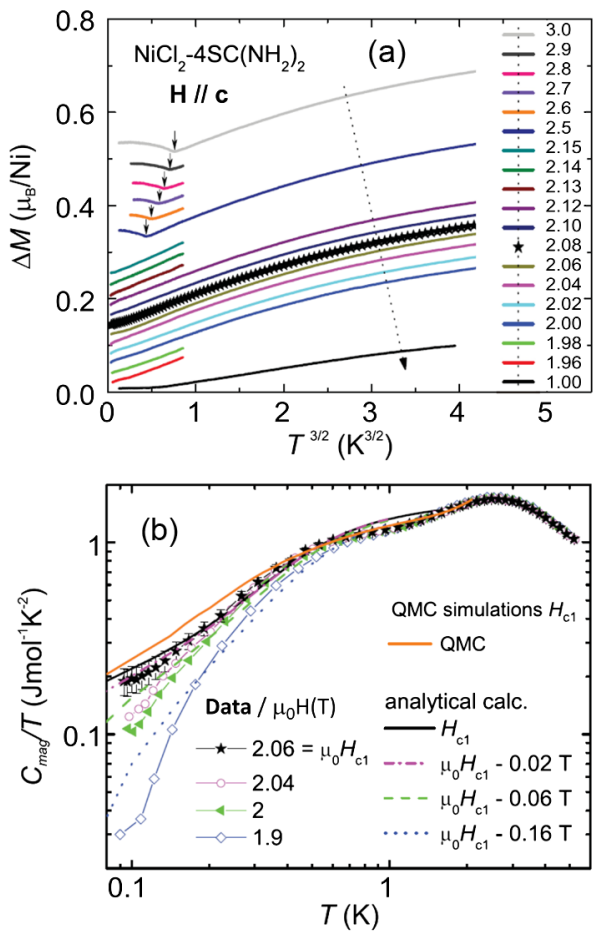

FIG. 30 (color online). (a) DTN $\left[\mathrm{NiCl}_{2}-4 \mathrm{SC}\left(\mathrm{NH}_{2}\right)_{2}\right]$ magnetization $M$, vs $T^{3 / 2}$ for magnetic field values between 1 and $3 \mathrm{~T}$. (b) Measured specific heat vs temperature compared against the results of QMC simulations From Weickert et al., 2012.

For $\mathrm{TlCuCl}_{3}$, studies of the thermal expansion and specific heat do not follow the expected universal behavior, although the Grüneisen parameter seems to be consistent with expected BEC-like behavior nevertheless. Small U(1)-symmetry-breaking DM terms were identified from ESR measurements of this compound (Glazkov et al., 2004). A staggered-field component perpendicular to the uniform applied field is induced by the small DM interaction. This staggered component opens a spin gap at the critical field $H_{c 1}$. The experimental observation is that the transition becomes first order due to lattice involvement (Vyaselev et al. 2004). This qualitative change in the lowenergy excitation spectrum at $H_{c 1}$ could explain the deviation from BEC predictions.

\section{Pressure-induced quantum critical points}

This article focuses primarily on magnetic-field-induced BECs. However, a different QCP can be reached by applying pressure to a quantum paramagnet. In this case, the gap is closed by lowering not just one spin level, as is the case with magnetic fields, but all of the excited levels that are connected by global spin rotations or time-reversal symmetries. Since both symmetries are preserved under the application of external pressure, the universality class of the pressure-induced QCP is different from the universality class of the field-induced QCP. For instance, pressure can close the gap of an isotropic spin dimer compound by increasing the ratio between the interdimer exchange and intradimer exchange constants. This is the case for $\mathrm{TlCuCl}_{3}$ (see Fig. 32), which develops AFM ordering above a critical pressure just over $1 \mathrm{kbar}$, according to neutron diffraction, and $0.042 \mathrm{kbar}$, according to magnetization
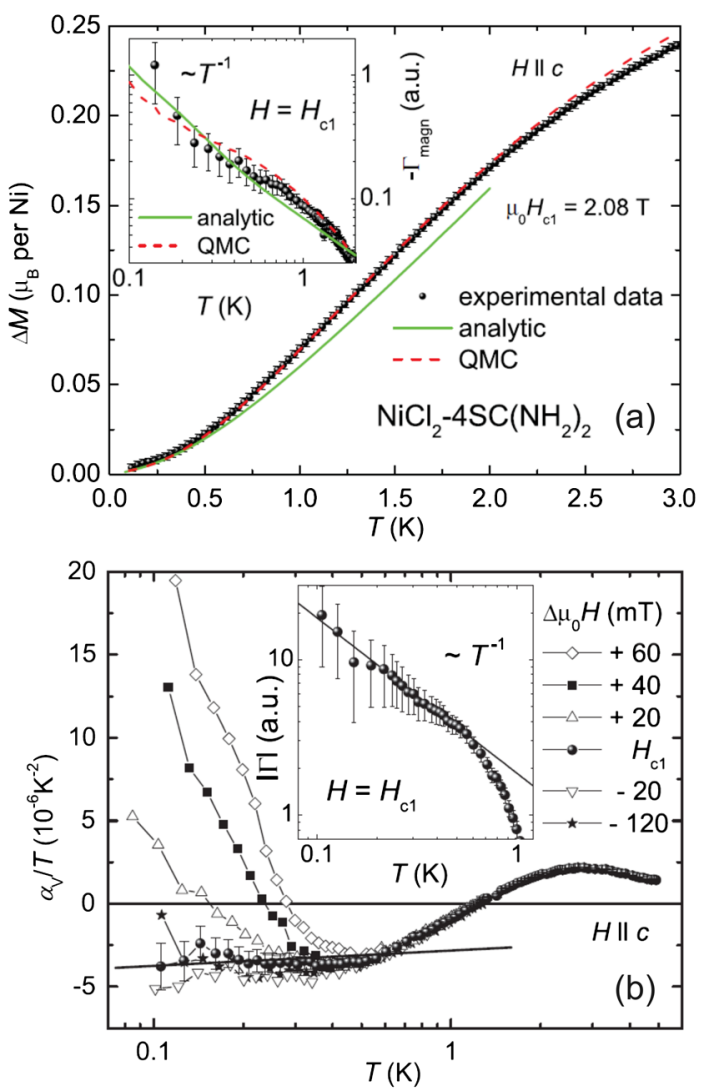

FIG. 31 (color online). (a) Measured magnetization $M$, vs temperature $T$ compared against the results of QMC simulations and analytical results for DTN $\left[\mathrm{NiCl}_{2}-4 \mathrm{SC}\left(\mathrm{NH}_{2}\right)_{2}\right]$. This comparison shows that the experimental data follow the expected behavior for $T \rightarrow 0$. Inset: $T^{-1}$ behavior of the magnetic Grüneisen parameter. (b) Coefficient of volumetric thermal expansion $\alpha$ divided by temperature as a function of $T$ for different magnetic fields. Inset: $T^{-1}$ behavior of the thermal Grüneisen parameter From Weickert et al., 2012.

measurements (Oosawa et al., 2003; Goto et al., 2004; Matsumoto et al., 2004; Oosawa et al., 2004; Rüegg et al., 2004, 2008; Yamada et al., 2008). In this case, the universality class of the pressure-induced QCP is $\mathrm{O}(3)$ in dimension $D=d+1$, because the $\mathrm{O}(3)$ symmetry is spontaneously broken beyond the QCP and the dynamical exponent is $z=1$. The three triplet modes become gapless at the QCP and have a linear dispersion $(z=1)$, in contrast to the quadratic dispersion of the $S^{z}=1$ single triplet mode that becomes gapless at the field-induced BEC QCP. Another important difference between the field- and pressure-induced QCPs is that the magnetic field couples to a conserved quantity (the total magnetization along the field direction), e.g., a quantity that commutes with the Hamiltonian, while the perturbation induced by pressure does not.

Although the three triplet modes become gapless at the pressure-induced QCP of isotropic quantum dimer systems, a gap reopens for the longitudinal mode inside the ordered state. The remaining two gapless modes are the Goldstone modes associated with the spontaneous breaking of two continuous symmetries. These modes were measured in $\mathrm{TlCuCl}_{3}$ with INS (Rüegg et al., 2004, 2008). 


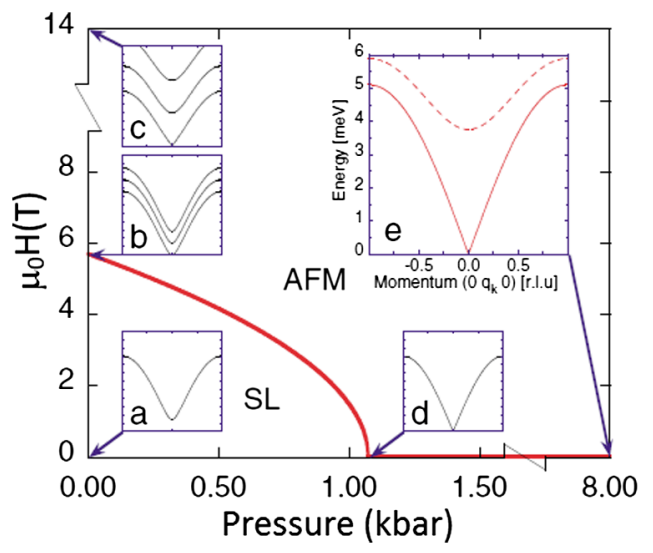

FIG. 32 (color online). Magnetic field vs pressure phase diagram for $\mathrm{TlCuCl}_{3}$ at $T=0 \mathrm{~K}$. A square root phase boundary separates the spin-liquid (SL) phase from the AFM phase. (a)-(e) Dispersion relation of triplet modes in for different temperatures and pressures. (a) Degenerate triplet modes at $H=0$ (anisotropies are neglected). (b) Zeeman-split triplet modes. (c) Spin dynamics in the field-induced ordered phase. (d) Degenerate and linear triplet modes close the spin energy gap at the critical pressure $P_{c}$. (e) Spin dynamics in the pressure-induced ordered phase. Two degenerate transverse Goldstone modes (solid line) and a longitudinal amplitude mode (dashed line) are expected From Rüegg et al., 2004.

Since $D=d+1$ for the pressure-induced QCP, mean-field behavior with logarithmic corrections is expected for $d=3$. This observation implies that the order parameter $\mathbf{M}_{\mathbf{Q}}$ is proportional to $\sqrt{P-P_{c}}$ and $T_{c} \propto \sqrt{P-P_{c}}$, where $P_{c}$ is the critical pressure. Pressure studies have also been done in IPA-CuCl ${ }_{3}$ (Hong et al., 2008) and PHCC (Hong et al., 2010) showing reductions in the spin gap, although these experiments could not reach the critical pressures to suppress the QCP to zero magnetic field.

If pressure studies were to be performed on a quantum magnet with uniaxial symmetry like DTN, one symmetry axis is already broken by the uniaxial crystal field. Thus, the pressure-induced QCP would belong to the $\mathrm{O}(2)$ universality class in dimension $D=d+1$. In this case, two gapless linear modes with $S^{z}= \pm 1$ are expected at the pressure-induced QCP because of time-reversal symmetry.

\section{Elastic neutron scattering}

Elastic neutron scattering (ENS) can directly probe the ordered magnetic moment in the field-induced ordered state. This ordered moment is proportional to the order parameter for the $X Y$ AFM. As explained before, the spins are always pinned along an easy axis inside the ordered phase of real magnets, so the description in terms of $X Y$-AFM ordering is always an approximation. Nevertheless it is important to understand the nature of the ordered phase, the direction of the easy axes, and the size of the ordered moments. ENS can confirm that there is a component of the ordered moment transverse to the applied magnetic field and test the power-law temperature dependences in the size of the ordered moment near the critical fields $H_{c 1}$ and $H_{c 2}$.
In a recent article, Grenier and Ziman, (2007) provided a comprehensive review of the use of modern neutron scattering techniques for the study of quantum magnetism with emphasis on the spin-ladder compound $\mathrm{NaV}_{2} \mathrm{O}_{5}$ and the spin dimer system $\mathrm{Cs}_{3} \mathrm{Cr}_{2} \mathrm{X}_{9}(X=\mathrm{Br}, \mathrm{Cl})$. We refer the interested reader to this work for more details on this technique. Here we provide an overview of ENS applied to quantum magnets with BEC QCPs, many of which are also spin-ladder compounds.

Tanaka et al performed ENS experiments on $\mathrm{TlCuCl}_{3}$ samples to confirm the transverse spin ordering and to investigate the temperature and field dependence of $m_{\perp}$ (Tanaka et al., 2001). Figure 33(a) shows the $\theta-2 \theta$ scans for the $(1,0,3)$ reflection measured at $T=1.9 \mathrm{~K}$ for $\mu_{0} H=0$ and $12 \mathrm{~T}$. Figure 33(b) shows the spin structure determined at $T=1.9 \mathrm{~K}$ and $\mu_{0} H=12 \mathrm{~T}$. The magnetic moments are ordered in the $a-c$ plane, which is perpendicular to the applied field, and they are antiparallel on the same dimers (represented by thick lines) forming an angle of $39^{\circ}$ with the $a$ axis. The spins on the same legs of the double chains located at the corner and the center of the unit cell in the $b$-c plane are antiparallel. The temperature dependence of the magnetic peak intensity at $(1,0,3)$ is shown in Fig. 33(c) for various applied magnetic fields. The square of the transverse magnetization per site $m_{\perp}^{2}$ is shown on the right $y$ axis. Thus, the transverse magnetic ordering predicted by the theory is confirmed by ENS experiments in $\mathrm{TlCuCl}_{3}$. ENS experiments were carried out in most other candidates for BEC behavior, confirming transverse ordering (see Table I). However, the spin ordering is not always a simple antiferromagnet transverse to the applied magnetic field. For instance, the frustrated compound Sul- $\mathrm{Cu}_{2} \mathrm{Cl}_{4}$ exhibits field-induced helical ordering (Garlea et al., 2009), whereas different ordered phases occur in $\mathrm{CsCu}_{2} \mathrm{Cl}_{4}$ depending on the field direction (Coldea et al., 2001), including a conical phase whose suppression was investigated in the context of BEC QCPs (Radu et al., 2005).

\section{Inelastic neutron scattering}

While elastic neutron scattering (ENS) provides direct information about the magnetic ordering, inelastic neutron scattering (INS) measures the dispersion relation of the magnetic excitations. The exchange constants are extracted from fits of the measured dispersion. Therefore, INS data are crucial for constructing minimal spin Hamiltonians. INS experiments also measure the quasiparticle dispersion inside the paramagnetic region that is invisible to ENS. Indeed, INS studies have been used to (a) measure the spin gap directly, (b) measure the dispersion of the triplon modes in the paramagnetic region, (c) produce essential information on the magnitude and sign of the relevant exchange interactions and potential anisotropies, (d) confirm that the spin gap can be closed with external parameters such as magnetic field or pressure, and (e) uncover the emergence of a magnetic Goldstone mode in the ordered state (at least to a good approximation). INS is also the ideal experimental tool for measuring spontaneous decays of high-energy magnons that commonly occur near magnetic-field-induced QCPs (Zhitomirsky and Chernyshev, 2013). Ideally INS is combined with complementary techniques, such as electronic paramagnetic spin resonance (EPR or ESR), that have higher 

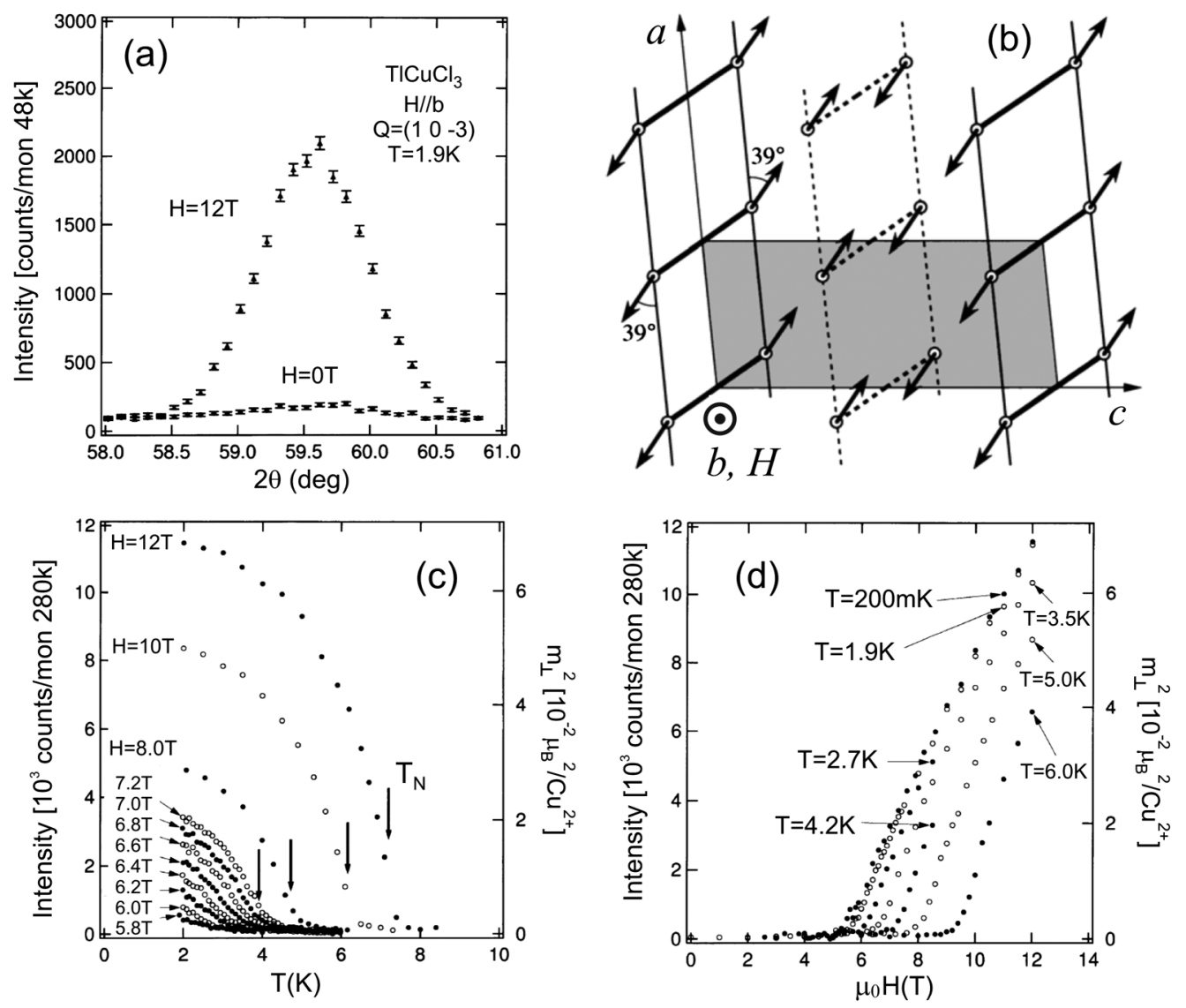

FIG. 33. $\mathrm{TlCuCl}_{3}$ elastic neutron diffraction study. (a) $\theta-2 \theta$ scans for $(1,0,3)$ reflection measured at $\mu_{0} H=0$ and $12 \mathrm{~T}$ at $1.9 \mathrm{~K}$. (b) Spin structure in the ordered phase. The external field is applied along the $b$ axis. The double chains located at the corner and the center of the chemical unit cell in the $b-c$ plane are represented by solid and dashed lines, respectively. The shaded area is the chemical unit cell in the $a-c$ plane. (c) Temperature dependence of the magnetic peak intensity at $(1,0,3)$ in various magnetic fields. The square of the transverse magnetization per site $m_{\perp}^{2}$ is shown on the right $y$ axis. (d) Magnetic field dependence of the magnetic peak intensity for (1,0,3) at various temperatures. The square of the transverse magnetization per site $m_{\perp}^{2}$ is shown on the right $y$ axis. From Tanaka et al., 2001.

energy resolution but can only access magnetic excitations with zero transferred momentum.

The importance of INS results is reflected in the large number of groups that have devoted resources to studying powder and single-crystal samples of quantum magnets that are strong candidates for the observation of field-induced BEC QCPs including but not limited to Sasago et al. (1997), Cavadini et al. (2001, 2002), Coldea et al. (2002), Coldea, Tennant, and Tylczynski (2003), Rüegg et al. (2003, 2004, 2007, 2008), Masuda et al. (2006), Stone et al. (2006, 2007, 2008b, 2008c), Zapf et al. (2006), Garlea et al. (2007, 2008), Zheludev et al. (2007, 2008), Hong et al. (2008, 2010), Kofu, Kim et al. (2009), Kofu, Ueda et al. (2009), Thielemann, Rüegg, Rønnow et al. (2009), Quintero-Castro et al. (2010), Schmidiger et al. (2011), and Tsyrulin et al. (2013).

The dispersion relation of the magnetic excitations has been measured for most of the quantum magnets that are discussed and the corresponding exchange constants were extracted from these measurements. For example, the zero-field triplon dispersion measured in $\mathrm{TlCuCl}_{3}$ is shown in Fig. 34. The dispersion relation of the different branches of magnetic excitations, $\omega_{\nu}(\mathbf{k})$ ( $\nu$ is the branch index) are extracted from the peaks (poles) of the measured dynamical structure factor $S(\mathbf{q}, \omega)$. The values of the exchange interactions and single-ion anisotropies are determined by fitting the measured curve with a model. In some cases, analytical approximations can be benchmarked with controlled numerical calculations of the spectrum of spin excitations. As discussed in Sec. II.D.4, the DMRG method can be used to compute the spectrum of spin excitations in one-dimensional systems. As long as the off-diagonal terms of the Hamiltonian are not frustrated, this spectrum can also be computed numerically for higherdimensional systems by means of quantum Monte Carlo simulations combined with maximum entropy methods (Sandvik, 1998). Typically, the generalized spin-wave approach described in Sec. II.D.2 works very well in spatial dimension $d \geq 2$, as long as the effective magnetic interactions are not strongly frustrated. Returning to Fig. 34 we note that the dispersion is quadratic in energy about the minimum. This gapped quadratic (massive) dispersion was derived in the theory sections and should persist up to $H_{c 1}$. The excitations become gapless and linear for $H_{c 1}<H<H_{c 2}$.

INS studies in single crystals of $\mathrm{Ba}_{3} \mathrm{Mn}_{2} \mathrm{O}_{8}$, reveal that not only the nearest-neighbor $(\mathrm{NN})$ but also the next-nearestneighbor (NNN) exchange interactions play an important role. Singlet-triplet dispersion relations show that the NN and NNN 

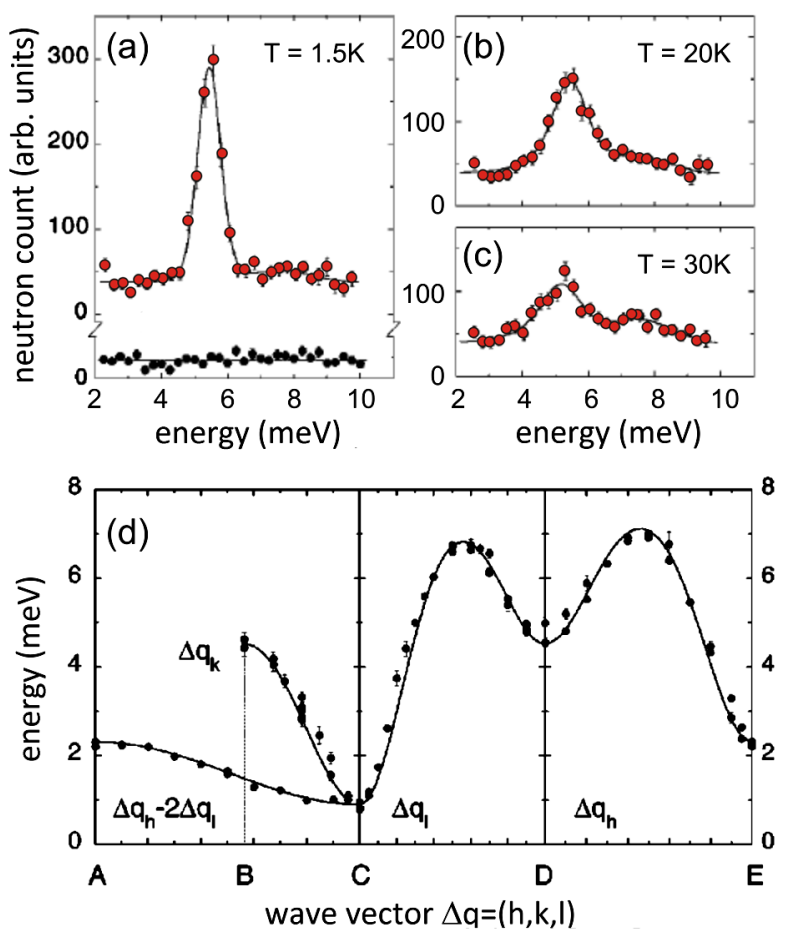

FIG. 34 (color online). (a)-(c) Measured neutron intensity vs energy transfer in the $a-c$ plane of $\mathrm{TlCuCl}_{3}$ for $(1.35,0,0)$, (top) and $(0,0,3.15)$ (bottom) (r.l.u.). (d) Observed energy dispersion of the magnetic excitation modes in $\mathrm{TlCuCl}_{3}$ at $T=1.5 \mathrm{~K}$. Full circles from the relevant directions of reciprocal space are arranged in a reduced scheme representation, with $A=E=(1 / 2,0,0), B=(0,1,0), D=(0,0,0)$ (r.l.u.). Zone centering corresponds to $C=(0,0,1)$ for $\Delta \mathbf{q}=(\mathrm{h}, 0,1), \mathrm{C}=$ $(0,0,0)$ for $\Delta \mathbf{q}=(0, \mathrm{k}, 0)$. From Cavadini et al., 2001. interactions are antiferromagnetic and compete between adjacent bilayers (Stone et al., 2008b). In particular, the existence of the NNN exchange interaction was unveiled by comparing the measured dispersion of the triplet excitation with the dispersion relation obtained with the generalized spin-wave approach described in Sec. II.D.2. The excellent agreement between theory and experiment (see Fig. 35) can be obtained only if the NNN interlayer exchange is included. Although the interlayer exchange is comparable to the intralayer exchange, this competition between the NN and NNN interlayer couplings reduces the effective magnetic coupling along the $c$ axis and leads to quasi-two-dimensional behavior. In addition, the four critical fields $H_{c 1}, H_{c 2}, H_{c 3}$, and $H_{c 4}$ that appear in the experimental phase diagram (see Fig. 8) are well reproduced with the exchange constants extracted from the fit of the INS dispersion shown in Fig. 35. Recall that this material consists of $S=1$ spins arranged in dimers, and thus not only total $S=1$ triplet excited states but also total $S=2$ quintuplet excited states are present. This compound was discussed in Secs. III.A and III.B.5 showing that the phase diagram has two dome-shaped regions of BEC where the triplet and the quintet become degenerate with the ground state (see Fig. 8).

INS studies of $\mathrm{Ba}_{3} \mathrm{Mn}_{2} \mathrm{O}_{8}$ at higher temperatures in polycrystals show the presence of the quintuplet excited state in addition to the triplet state. INS data for this material (Fig. 36) show the presence of both singlet-triplet and singlet-quintet excitations in plots of the intensity versus energy transfer $\hbar \omega$. The higher-energy singlet-quintet excitations become more noticeable as the temperature is increased, appearing as small shoulders near $\hbar \omega=3 \mathrm{meV}$. By contrast, the peak due to the lower-energy singlet-triplet excitations just below $2 \mathrm{meV}$ is suppressed with increasing temperature.

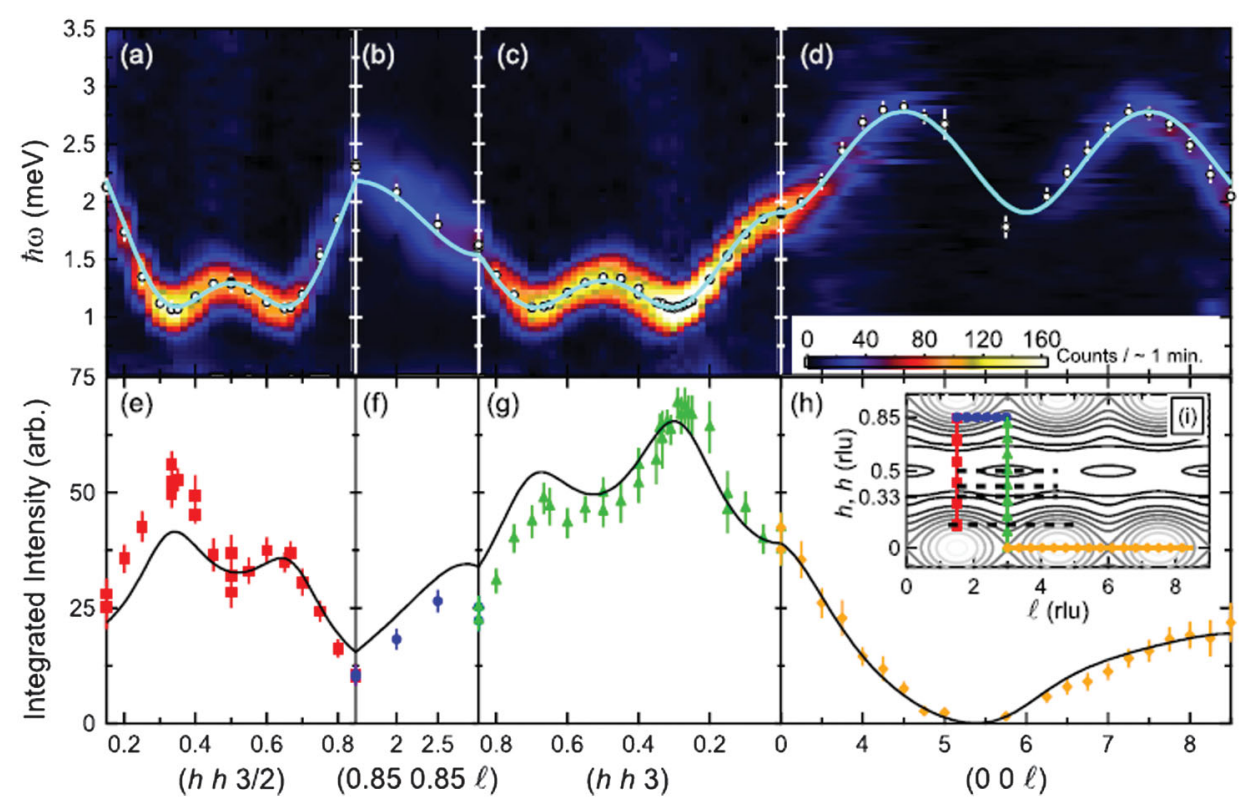

FIG. 35 (color). $\quad T=1.7 \mathrm{~K}$ inelastic neutron scattering data for $\mathrm{Ba}_{3} \mathrm{Mn}_{2} \mathrm{O}_{8}$, which have six degenerate minima (only one shown) in the dispersion relation. (a)-(d) Scattering intensity (contour plot) and $\hbar \omega$ (points) vs $\mathbf{Q}$ plotted in reciprocal lattice units (r.l.u.). Solid lines are fits to a calculated dispersion relation. (e)-(h) Integrated scattering intensity from Gaussian fits to constant $\mathbf{Q}$ scans. Solid lines are fits. (i) Path through the $(h h l)$ plane shown in (a)-(h). From Stone et al., 2008b. 


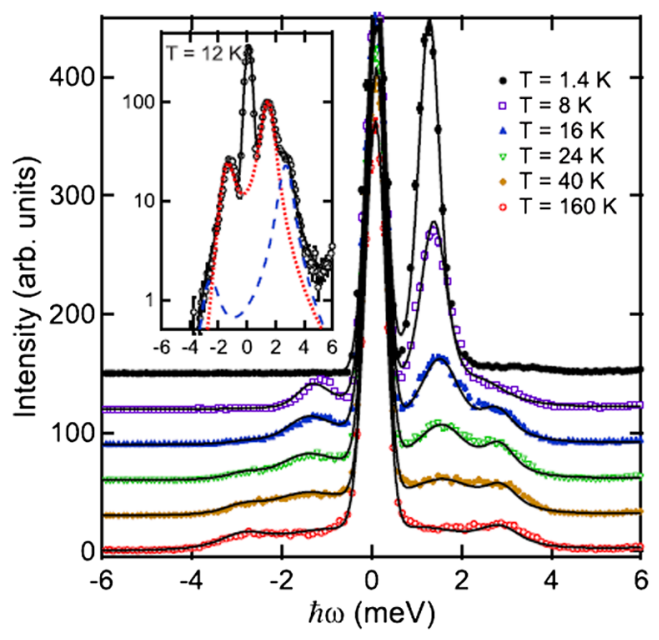

FIG. 36 (color online). Energy-dependent scattering intensity of $\mathrm{Ba}_{3} \mathrm{Mn}_{2} \mathrm{O}_{8}$ for $0.9<Q<1.0 \AA^{-1}$ for (from top to bottom): $T=1.4,8,16,24,40$, and $160 \mathrm{~K}$. Data for different temperatures are vertically offset. Solid lines are fits to Lorentzian functions convolved with the energy-dependent resolution of the instrument. The inset illustrates scattering intensity of the $T=12 \mathrm{~K}$ measurement plotted on a logarithmic intensity scale. The dotted line corresponds to the spectral contribution of the lower-energy singlet-triplet mode, and the dashed line corresponds to the higher-energy singlet-quintet mode. From Stone et al., 2008c.

Having described INS measurements of the zero-field gapped state, we can extract even more information by applying magnetic fields to suppress the gap. Turning once again to $\mathrm{TlCuCl}_{3}$ as an example, Fig. 37 shows the evolution of the spin triplet states in applied magnetic fields. The lowest level of the spin triplet becomes degenerate with the spin singlet near $6 \mathrm{~T}$. Inside the ordered phase, the gap in principle should not reopen, and the lowest excitation should be a gapless Goldstone mode with linear dispersion. For $\mathrm{TlCuCl}_{3}$, INS data are shown at $14 \mathrm{~T}$, well inside the ordered phase, and a mode is indeed found whose dispersion can be fit to a linear function (Rüegg et al., 2003). Again, we note that small U(1)symmetry-breaking terms discussed in Sec. II.B should open a small spin gap inside the ordered phase. However, this gap may not be observable with the available energy resolution of state-of-the-art INS experiments.

In Sul- $\mathrm{Cu}_{2} \mathrm{Cl}_{4}$, INS reveals not only the (mostly) gapless Goldstone mode inside the ordered phase but an additional gapped mode with a gap that opens inside the ordered phase, reaching a maximum energy near $0.3 \mathrm{meV}$ (Zheludev et al., 2009). This gapped mode is not due to uniaxial-symmetrybreaking terms (after all, the mostly gapless mode is also observed), but it is consistent with theoretical predictions for this frustrated spin-ladder material. Similarly, in DTN, a gapped mode opens in addition to the (mostly) gapless mode, although for completely different reasons. This gapped mode has been observed in ESR (Zvyagin et al., 2007) and INS in magnetic fields (Tsyrulin et al., 2013). It is attributed to the fact that DTN has two interpenetrating tetragonal lattices with weak frustrated coupling between them. This weak coupling lifts the degeneracy between the two Goldstone modes of the decoupled sublattices, leading to one gapped mode and a remaining global Goldstone mode.

INS provides us not only with the information necessary to analyze BEC QCPs in these quantum magnets, but it has also uncovered a rich variety of other behavior in several quantum magnets in this paper. For example, a large body of work on $\mathrm{CsCu}_{2} \mathrm{Cl}_{4}$ has interpreted the INS data on that compound as evidence for fractionalized excitations due to a resonantvalence-bond model, which generated much excitement for a while. Recent work, however, reinterprets the data as arising from a hierarchy of energy scales created by frustration, in which very small interlayer exchange and DM interactions can play a dominant role in shaping the magnetic behavior (Starykh, Katsura, and Balents, 2010). We refer the interested reader to a recent review on this subject (Balents, 2010). In another compound, PHCC (Stone et al., 2006), INS data show a breakdown of the quasiparticle description that is normally used to describe the magnon excitations between the nonmagnetic ground state and the excited state in gapped quantum magnets. In PHCC, the quasiparticle dispersion terminates above a critical wave vector and merges with the two-particle continuum. This is similar to what is observed in excitations of superfluid ${ }^{4} \mathrm{He}$. This behavior can be traced to
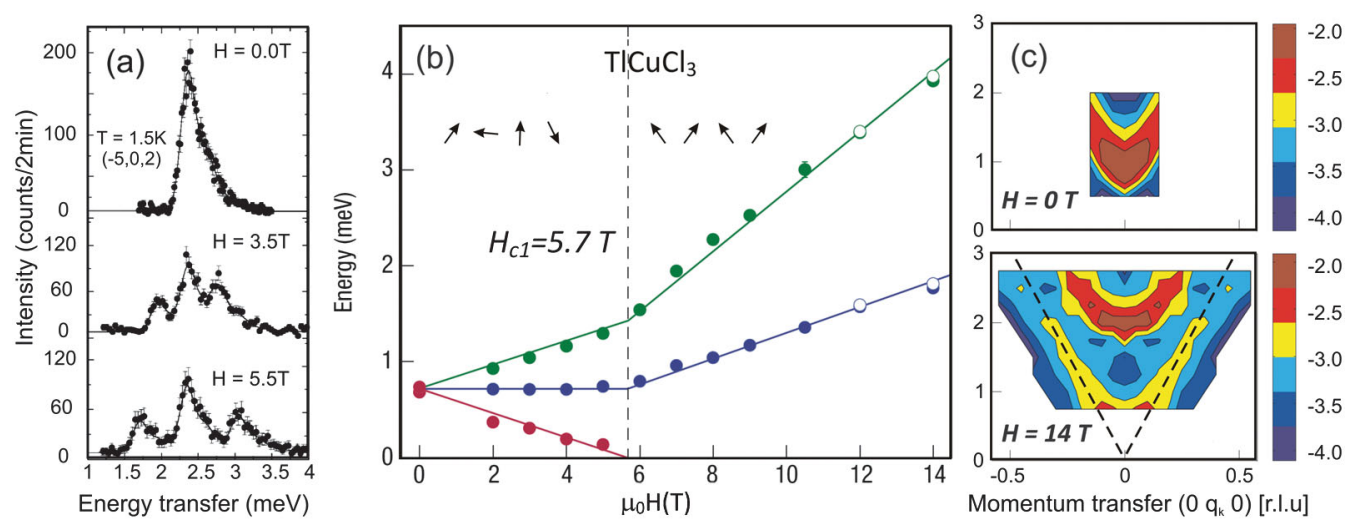

FIG. 37 (color). $\quad \mathrm{TlCuCl}_{3}$ inelastic neutron diffraction study. (a) Intensity vs energy transfer showing the emergence of three spin levels with different energies (Furrer and Rüegg, 2006). (b) Spin levels as a function of external magnetic field $H$. (c) Contour plot of the neutron scattering intensity vs momentum transfer, in the spin gapped state at $H=0$ (top), and inside the ordered phase at $H=14 \mathrm{~T}$ showing the linear dispersion of the Goldstone mode. From Rüegg et al., 2003. 

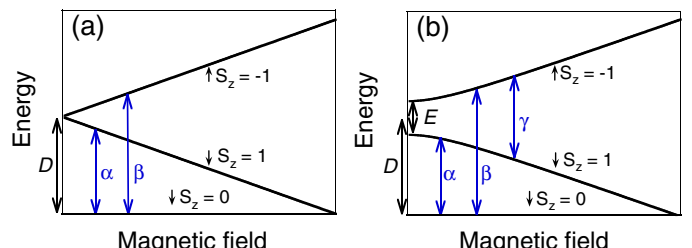

FIG. 38 (color online). (a) Energy-level diagram for an $S=1$ spin triplet with a Hamiltonian $\mathcal{H}=g \mu_{B} H_{z} S^{z}$ that obeys uniaxial symmetry. The transitions $\alpha$ and $\beta$ obey the $\Delta S^{z}= \pm 1$ selection rules and would be observed in ESR. (b) $\mathcal{H}$ contains additional terms $D\left(S^{z}\right)^{2}+E\left[\left(S_{x}\right)^{2}-\left(S_{y}\right)^{2}\right]$. The $E$ term breaks the continuous uniaxial symmetry and disallows BEC for $k_{B} T<E$. The $D$ term breaks uniaxial symmetry only if $H$ is not parallel to the $z$ axis. The eigenstates of the new Hamiltonian now contain mixtures of the different $S^{z}$ states; thus the energies are no longer linear in $H$ and follow $E_{ \pm}=D \pm \sqrt{\left(2 g \mu_{B} H\right)^{2}+E^{2} / 4}$.

the highly frustrated nature of the magnetic interactions in PHCC in the predominantly $2 \mathrm{D}$ planes of dimers.

\section{Electron spin resonance}

The ESR technique is critically important for identifying uniaxial-symmetry-breaking terms in quantum magnets. ESR is based on the resonant absorption of electromagnetic energy due to transitions between different spin levels. It is a very sensitive probe for detecting transitions between spin states that have the same momentum and whose energy difference is in the range of a fraction of a kelvin up to a few tens of kelvins. In the presence of magnetic exchange interactions that disperse the spin levels, ESR couples to the ferromagnetic $(k=0)$ edge of each dispersed spin level since the applied magnetic field is uniform.

Figure 38 shows spin levels evolving in magnetic field for an $S=1$ isolated spin, or alternatively for the $S=1$ triplet of an $S=1 / 2$ dimer. Since ESR results from Zeeman coupling to a time-dependent magnetic field that is perpendicular to the static magnetic field, it is subject to the selection rule $\Delta S^{z}= \pm 1$ (neglecting orbital contributions). The figure on the left shows the case of a system with perfect uniaxial symmetry, while the figure on the right corresponds the case in which the uniaxial anisotropy is broken by the additional terms $D\left(S^{z}\right)^{2}+E\left[\left(S^{x}\right)^{2}-\left(S^{y}\right)^{2}\right]$. For an isolated spin, $D$ and $E$ are single-ion anisotropy terms that result from interaction between the spin and its orbit, which is affected by the crystal electric field of surrounding atoms. For a dimer, however, the effective $D$ and $E$ terms that split the triplet levels arise from intradimer symmetric exchange anisotropy and dipole-dipole interactions. The $D$ term leaves a single uniaxial symmetry axis. Therefore, uniaxial symmetry is preserved in the presence of an external field $\mathbf{H}$ only if $\mathbf{H}$ is parallel to the symmetry axis. The $D$ term splits the $S^{z}=0$ and $S^{z}= \pm 1$ states as shown in Fig. 38. The $E$ term splits the $S^{z}= \pm 1$ states and breaks uniaxial symmetry because it does not commute with $S^{z}$, i.e., the Hamiltonian eigenstates are linear combinations of eigenstates of $S^{z}$ that have different eigenvalues. The degree of mixing decreases with $H$, because the Zeeman term opens a gap between states with different $S^{z}$ that

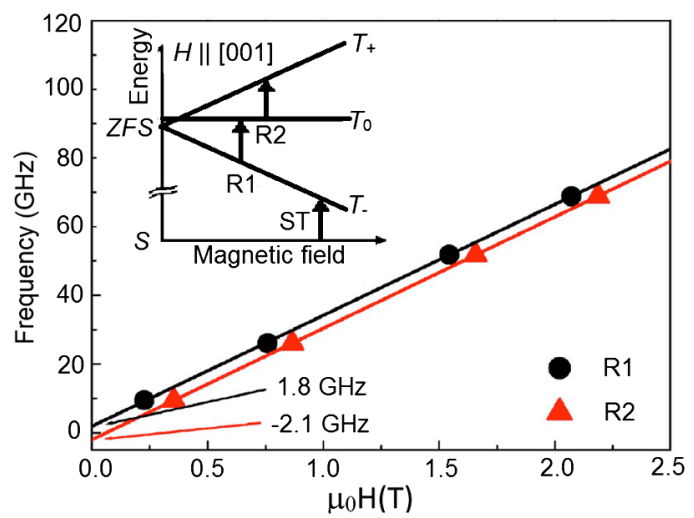

FIG. 39 (color online). ESR measurements on $\mathrm{BaCuSi}_{2} \mathrm{O}_{6}$ single crystals at $T=0.6 \mathrm{~K}$. The frequencies of intratriplet splittings of the $\mathrm{Cu}$ dimer $S=1$ triplet are plotted as a function of $\mathbf{H} \| \hat{c}$, labeled R1 and R2, showing linear evolution with a very small offset from zero, from which the maximum size of $D$ can be estimated. There is no evidence for a nonzero $E$ in these data. The inset shows a cartoon of the triplet levels evolving in magnetic field. From Sebastian, Tanedo et al., 2006.

increases linearly in $H$. Thus, the energy levels evolve nonlinearly at low magnetic fields, and linearly for fields $H \gg E / g \mu_{B}$. Therefore, the magnitude of the U(1)-symmetrybreaking term can be estimated from the size of the region in which the excited spin levels do not evolve linearly in magnetic field, and from the size of the zero-field gaps between spin levels. This is true not only for the examples that we used to illustrate the point, but for any other magnetic system: U(1)-symmetry-breaking terms mix states with different total $S^{z}$ and consequently lead to nonlinear dependence of the energy as a function of $H$. U(1) symmetry-breaking terms can also arise from interdimer interactions, in addition to intradimer interactions, and from interactions between nondimerized spins. DM interactions between spins will break the $\mathrm{U}(1)$ symmetry if $\mathbf{H}$ is not parallel to the DM vector $\mathbf{D}_{\mathrm{DM}}$. DM interactions $\mathbf{D}_{\mathrm{DM}} \cdot \mathbf{S}_{1} \times \mathbf{S}_{2}$ can be readily detected in spin dimer systems by looking for singlet-triplet transitions, which become allowed due to the antisymmetric nature of the DM coupling under exchange of both spins $1 \Leftrightarrow 2$ (note that the triplet and the singlet have opposite parity under this transformation).

ESR experiments have been performed in a number of BEC compounds (Schrama et al., 1998; Takatsu et al., 1998; Tanaka et al., 1998; Manaka et al., 2001; Kodama et al., 2005; Zvyagin et al., 2007, 2008; Cox et al., 2008; Fujisawa et al., 2009; Kofu, Ueda et al., 2009; Povarov et al., 2011; Glazkov et al., 2012; Psaroudaki et al., 2012). Some of these experiments have measured the size of the gap between pairs of spin levels and the degree of linearity as a function of magnetic fields, thus probing uniaxial symmetry down to the lowest energy scale that can be resolved with this technique. The measured U(1)-symmetry-breaking terms are listed in Table I for the various materials discussed in this paper.

An analysis of ESR measurements in $\mathrm{BaCuSi}_{2} \mathrm{O}_{6}$ (Sebastian, Tanedo et al., 2006) found a zero-field splitting (ZFS) of the triplet states that is consistent with a symmetric exchange anisotropy of $0.1 \mathrm{~K}$. This term does not break U(1) 
symmetry along the dimer axis and arises from intradimer dipole-dipole interactions. The ESR data are shown in Fig. 39. The absence of splitting between the $S^{z}= \pm 1$ states and the lack of singlet-triplet transition indicate that the effect of U(1)symmetry-breaking terms, such as dipolar interactions between different dimers, is smaller than the experimental resolution. Indeed, the effective U(1)-symmetry-breaking term produced by interdimer dipolar interactions is estimated to be of the order of $10 \mathrm{mK}$, i.e., $0.02 \%$ of the maximum ordering temperature $T_{c \max }$.

ESR measurements have found more significant deviations from uniaxial symmetry in $\mathrm{TlCuCl}_{3}$ (Glazkov et al., 2004), PHCC (Glazkov et al., 2012) and $\mathrm{CsCu}_{2} \mathrm{Cl}_{4}$ (Povarov et al., 2011). Symmetry exchange anisotropies are evident from the ESR data of $\mathrm{TlCuCl}_{3}$ and PHCC. In addition, the singlet-triplet transition observed in $\mathrm{TlCuCl}_{3}$ clearly indicates the presence of $\mathrm{U}(1)$-symmetry-breaking DM interactions. The order of magnitude of these anisotropic terms in $\mathrm{TlCuCl}_{3}$ is estimated to be $1 \%$ of the maximum ordering temperature. In PHCC, $D=$ $0.37 \mathrm{~K}$ and $E=0.06 \mathrm{~K}$, which is $5 \%$ and $0.8 \%$, respectively, of the maximum ordering temperature. In $\mathrm{CsCu}_{2} \mathrm{Cl}_{4}, \mathrm{DM}$ interactions along two different crystallographic axes were identified so there is no configuration in which $\mathbf{H}$ is parallel to $\mathbf{D}_{\mathrm{DM}}$. The energies of the DM interactions are reported to be 0.38 and $0.52 \mathrm{~K}$, which is $63 \%$ and $87 \%$ of the $0.6 \mathrm{~K}$ ordering temperature (Povarov et al., 2011). This analysis is inconsistent with $\mathrm{BEC}$ in $\mathrm{Cs}_{2} \mathrm{CuCl}_{4}$.

The ESR data for DTN (Zvyagin et al., 2007, 2008), IPA- $\mathrm{CuCl}_{3}$ (Manaka et al., 2001), $\mathrm{Ba}_{3} \mathrm{Cr}_{2} \mathrm{O}_{8}$ (Kofu, Ueda et al., 2009), and $\mathrm{Sul}_{-} \mathrm{Cu}_{2} \mathrm{Cl}_{4}$ (Fujisawa et al., 2009) are consistent with uniaxial symmetry for at least one field direction in each case, although the precise upper bound for U(1)-symmetry-breaking terms is not estimated from the data. In DTN there is a dominant $D$ term along the crystallographic $c$ axis, thus $\mathbf{H}$ must be parallel to $c$ to preserve $\mathrm{U}(1)$. There is a DM interaction $\mathbf{D}_{\mathrm{DM}} \perp c$ in $\mathrm{Ba}_{3} \mathrm{Cr}_{2} \mathrm{O}_{8}$, so $\mathbf{H}$ must be perpendicular to $\hat{c}$ in order to preserve $\mathrm{U}(1)$ the symmetry.

ESR measurements also reveal the $g$ factor, whose deviations from the free electron value $\sim 2$ give a rough estimate of the ratio between the spin-orbit coupling and the crystal field splitting between $3 d$ levels of the transition metal ion. In the case of IPA- $\mathrm{CuCl}_{3}$, a 3D mapping of the $g$ factor allowed them to derive the arrangement of the $\mathrm{Cu} d_{x^{2}-y^{2}}$-like orbitals that contribute to the magnetic exchange interactions (Manaka, Yamada, and Higemoto, 2007).

Finally, ESR was used in DTN to observe a two-magnon bound state above the saturation field (Fig. 40). The single magnon excitation above $H_{c 1}$ corresponds to an $\left|S^{z}=0\right\rangle$ state that propagates on top of the fully polarized ground state with well-defined momentum. The large $D / J_{c}$ value in DTN leads to the formation of two-magnon bound states. The $\mathbf{k}=\mathbf{0}$ twomagnon bound state has been detected with ESR (Zvyagin et al., 2007) in spite of the fact that $\Delta S^{z}=-2$. The origin of this violation of the selection rule $\Delta S^{z}= \pm 1$ is still unclear, although it could be explained by small misalignment between the applied field and the crystallographic $c$ axis in that measurement, or by DM terms. $T=0.4 \mathrm{~K}$ measurements in this material (Zvyagin et al., 2008) also reveal an energy gap opening in the ordered phase. This gap on first sight could be interpreted as evidence of uniaxial symmetry breaking.
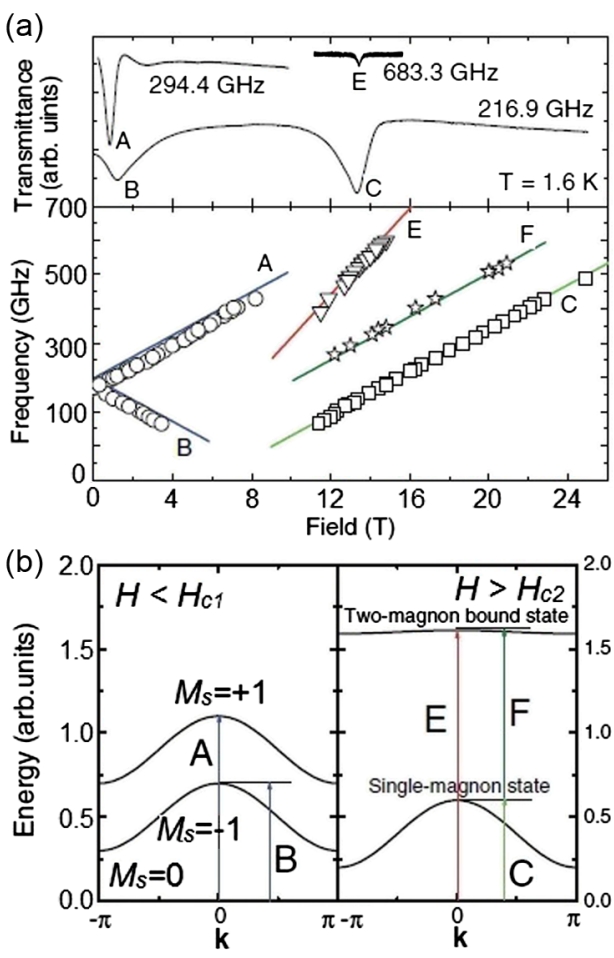

FIG. 40 (color online). Electron spin resonance data at $1.4 \mathrm{~K}$ for single crystals of DTN $\left[\mathrm{NiCl}_{2}-4 \mathrm{SC}\left(\mathrm{NH}_{2}\right)_{2}\right]$. (a) Top: resonant absorption of energy at a fixed frequency as a function of magnetic field. Bottom: magnetic field dependence of the resonant absorption lines labeled A-F. (b) Schematic of the five absorption lines A-F, which include not only resonant transitions between spin levels (A and B), but also single- and two-magnon bound states $(\mathrm{C}, \mathrm{E}, \mathrm{F})$. The expected dispersion of the spin levels are shown, which illustrated the fact that ESR measures ferromagnetic (uniform field) excitations, which correspond to the top (energy maximum) of the dispersion in DTN because the interactions are antiferromagnetic. From Zvyagin et al., 2007.

However, Zvyagin et al. (2008) found that the relative sizes of the resonances in the ESR data and the field dependence of the gap size are very inconsistent with that scenario. Rather, the data are consistent with a coupling between the low-energy modes of the two interpenetrating simple tetragonal sublattices of DTN. The finite coupling between the two sublattices splits the two gapless modes into a gapped mode, in which the spins of both sublattices rotate in opposite directions, and a (mostly) gapless Goldstone mode associated with a global spin rotation in which the spins of both sublattices rotate by the same amount.

\section{Nuclear magnetic resonance}

NMR provides a sensitive probe of the local magnetism and can yield important details about these quantum magnets exhibiting BEC. In particular, it becomes a very valuable technique for quantum paramagnets whose values of $H_{c 1}$ are above $20 \mathrm{~T}$. The difficulty of doing neutron scattering experiments under such high fields (at least for the moment) leaves NMR as the primary probe for revealing details of the magnetic order parameter that appears above $H_{c 1}$. Such experiments are particularly important for frustrated materials in which there are several competing order parameters right 
above $H_{c 1}$ because the single-boson dispersion has global minima at more than one wave vector (see Sec. II.D.3). The power-law dependence of the NMR relaxation rate $\left(1 / T_{1}\right)$ can also be used to test predictions in different regimes of the phase diagram (Giamarchi and Tsvelik, 1999; Orignac, Citro, and Giamarchi, 2007; Mukhopadhyay et al., 2012).

NMR measurements of the ${ }^{135,137} \mathrm{Ba}$ nucleus in $\mathrm{Ba}_{3} \mathrm{Mn}_{2} \mathrm{O}_{8}$ reveal a magnetization versus temperature curve at $H=H_{c 1}$, $\mathbf{M}\left(\mathbf{H}=\mathbf{H}_{c 1}, T\right)$ that is in agreement with the BEC scenario for $\mathbf{H} \| c$, but not $\mathbf{H} \perp c$ (Suh et al., 2011). This is expected because the Mn $S=3 / 2$ spins have a small uniaxial symmetric anisotropy ( $D$ term in the Hamiltonian) and thus $\mathbf{H}$ must lie along the $c$ direction for uniaxial symmetry to be preserved. The double-horn shape of the NMR line measured for $\mathbf{H} \| c$ and $H>H_{c 1}$ indicates that the magnetic ordering wave vector is incommensurate, as expected from the position of the minima of measured triplon dispersion (Stone et al., 2008b).

NMR measurements in $\mathrm{TlCuCl}_{3}$ show that the transition into the BEC phase is not purely second order, due to very strong spin-lattice coupling (Vyaselev et al., 2004). Locally the transition is discontinuous, indicative of a first-order component to the ordering. However, Vyaselev et al. (2004) note that bulk probes like INS can still show a seemingly continuous transition if the local value of $T_{N}$ varies somewhat across the sample.

NMR in $\mathrm{BaCuSi}_{2} \mathrm{O}_{6}$ reveals interesting structural complications (Krämer et al., 2007, 2013). ${ }^{63,65} \mathrm{Cu}$ and ${ }^{29} \mathrm{Si}$ NMR (Krämer et al., 2007) show that below $90 \mathrm{~K}$ at least two structurally different planes of $\mathrm{Cu}$ dimers form, with different intradimer exchange couplings and different gaps $A / B=1.16$. These data are shown in Fig. 41 and are mostly consistent with the INS measurements discussed in Sec. III.B.10 (Rüegg et al., 2007). The boson density near the critical field $H_{c 1}$ is five times higher in one plane than the other. The NMR data also show a broadening of the line widths that is consistent with an incommensurate structural modulation within the planes, which was also observed in high-resolution x-ray diffraction data (Samulon et al., 2006). Most recently, an even more detailed NMR study (Krämer et al., 2013) finds that frustration is not perfect, leading to partial condensation in the planes with lower boson densities. These findings have lead to several new theoretical approaches to understanding BECs in heterogeneous and frustrated systems (Rösch and Vojta, 2007; Laflorencie and Mila, 2009). We note that a recent study shows that the effective intralayer exchange is ferromagnetic for $\mathrm{BaCuSi}_{2} \mathrm{O}_{6}$, implying that there is no geometric frustration in this compound (Mazurenko et al., 2014). Thus the details of magnetic ordering in $\mathrm{BaCuSi}_{2} \mathrm{O}_{6}$ present an ongoing puzzle.

\section{MAGNETIC FRUSTRATION}

Since magnetic frustration is quite common in the quantum magnets we are reviewing, we highlight it with its own section. Frustration occurs in magnetic systems that cannot satisfy all their magnetic interactions even in the limit of classical moments $S \rightarrow \infty$ for which spin operators commute with each other. For example, a triangle of three antiferromagnetically coupled spins is frustrated because the spins
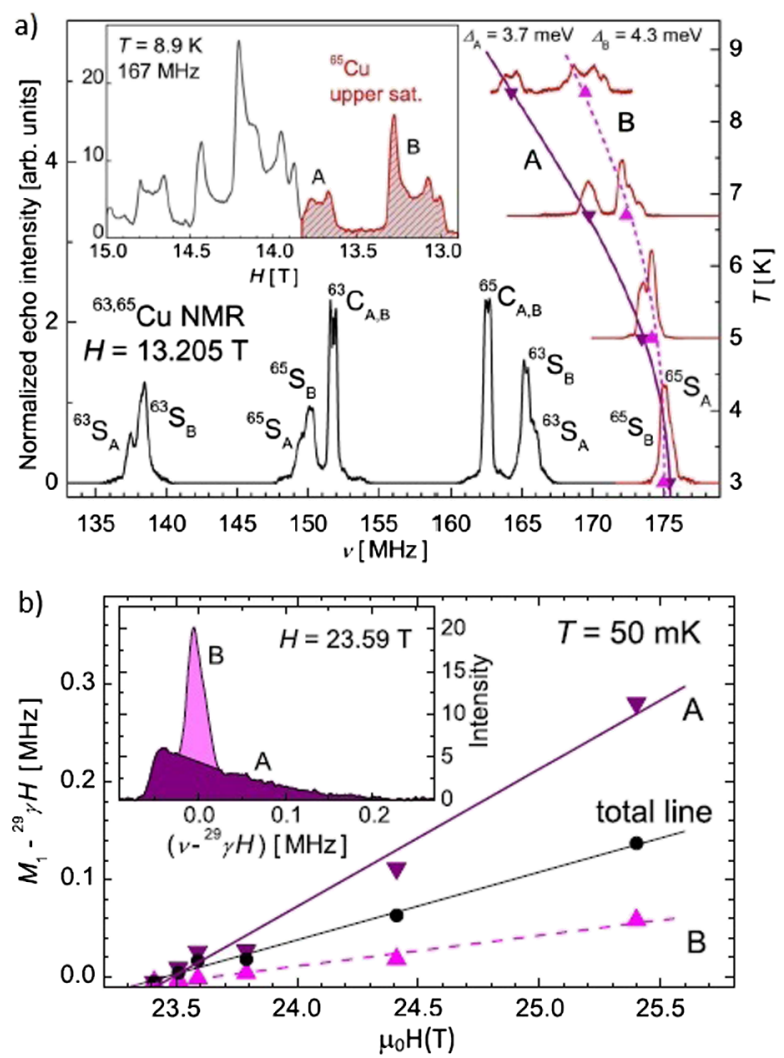

FIG. 41 (color online). (a) ${ }^{63,65} \mathrm{Cu} \mathrm{NMR}$ spectra of $\mathrm{BaCuSi}_{2} \mathrm{O}_{6}$ single crystals in the gapped phase $H<H_{c 1}$. The $T$ dependence of the high-frequency satellite reveals two different $\mathrm{Cu}$ sites associated with two different types of planes in the crystalline structure. Inset: field-sweep spectrum that reveals the incommensurate nature of the line shape for each of the two sites. Shading separates the contribution of the ${ }^{65} \mathrm{Cu}$ high-frequency satellite from the rest of the spectrum. The analysis of the latter part confirms that the observed line shape has a purely magnetic origin. (b) ${ }^{29} \mathrm{Si} \mathrm{NMR}$ at $50 \mathrm{mK}$ near $H_{c 1}$. A decomposition of the line shape into A and $\mathrm{B}$ as shown yields the different magnetizations of the two types of planes $\mathrm{A}$ and $\mathrm{B}$. This corresponds to a ratio of the average boson density $n_{A} / n_{B} \sim 5$. From Krämer et al., 2007.

cannot all simultaneously antialign with their neighbors. Frustration has different manifestations in the class of compounds that we are considering. This is particularly true for the dimerized quantum paramagnets. On the one hand, as discussed in Sec. II.D.1, frustration between exchange constants connecting the same pair of dimers (see Fig. 3) reduces the ratio between the hopping amplitude and the strength of the density-density repulsion in the effective low-energy model that describes the system as a gas of hard-core bosons [see Eqs. (31)]. On the other hand, frustration between exchange constants connecting different pairs of dimers can lead to more than one global minimum in the dispersion relation of the single-particle excitations for $H \leq H_{c 1}$. As we will see below, these two different manifestations of geometric frustration lead to different types of field-induced magnetic orderings.

$\mathrm{SrCu}_{2}\left(\mathrm{BO}_{3}\right)_{2}$ provides a clear example of suppression of the bosonic hopping amplitude due to frustration between exchange constants connecting the same pair of dimers (Kageyama, Yoshimura et al., 1999). In the notation of 
Sec. II.D.1, the exchange constants between two nearestneighbor dimers with coordinates $\mathbf{r}$ and $\mathbf{r}^{\prime}$ satisfy the following condition: $J_{11}^{\mathbf{r}, \mathbf{r}^{\prime}}=J_{12}^{\mathbf{r}, \mathbf{r}^{\prime}}$ and $J_{22}^{\mathbf{r}, \mathbf{r}^{\prime}}=J_{21}^{\mathbf{r}, \mathbf{r}^{\prime}}=0$. According to Eqs. (31), this condition implies that the bosons (triplets) cannot hop to first order in $J_{\nu \nu^{\prime}}^{\mathbf{r}, \mathbf{r}^{\prime}}$ because $t_{\mathbf{r}, \mathbf{r}^{\prime}}=0$. The effective hopping amplitude turns out to be of sixth order in the interdimer exchange (Fukumoto, 2000; Knetter et al., 2000; Totsuka, Miyahara, and Ueda, 2001). This observation explains the flat dispersion of the single-triplet excitations that has been observed with INS experiments (Kageyama et al., 2000). It also explains the existence of multiple fieldinduced magnetization plateaus (Smith and Keszler, 1991; Kageyama, Onizuka et al., 1999; Kageyama, Yoshimura, 1999; Misguich, Jolicoeur, and Girvin, 2001; Kodama et al., 2002; Shastry and Kumar, 2002; Miyahara and Ueda, 2003; Sebastian et al., 2008; Jaime et al., 2012). These plateaus correspond to different crystallizations of triplets, whose particular complex structures are difficult to determine for the lower magnetization steps due to the presence of multiple competing length scales.

As explained in Sec. II.C.2, frustration between exchange constants connecting different pairs of dimers can reduce the dimension of the field-induced BEC QCP. This drop in dimensionality, shown in Fig. 27, is very unusual, since dimensionality usually increases when approaching a critical point.

The most common consequence of frustration between exchange constants connecting different pairs of dimers is the emergence of spiral (or single-Q) and multiple-Q spin orderings right above the critical field $H_{c 1}$. As explained in Sec. II.D.3, the existence of more than one global minimum in the dispersion relation of single-triplet excitations implies that these bosons can condense in more than one singleparticle state. Condensation in a single single- $\mathbf{Q}$ state corresponds to a spiral phase. Condensation in a linear combination of different $\mathbf{Q}$ states leads to richer structures. In contrast to the case of single- $\mathbf{Q}$ ordering, the longitudinal spin component of multi-Q structures is always modulated. In other words, $X Y$ and Ising-like orderings coexist in the same phase.

$\mathrm{Ba}_{3} \mathrm{Mn}_{2} \mathrm{O}_{8}, \mathrm{Sul}-\mathrm{Cu}_{2} \mathrm{Cl}_{4}$, and $\mathrm{Cs}_{2} \mathrm{CuCl}_{4}$ are three examples where frustration leads to spiral structures with incommensurate wave vectors. In $\mathrm{Cs}_{2} \mathrm{CuCl}_{4}, \mathrm{Cu}^{2+} S=1 / 2$ spins form an anisotropic triangular lattice, which leads to helical ordering. In this material the choice of handedness of the helix is determined by the lattice, which already breaks inversion symmetry as evidenced by the existence of DM interactions. In contrast, the choice of handedness is spontaneous for the helix that is observed in Sul- $\mathrm{Cu}_{2} \mathrm{Cl}_{4}$ (a four-leg spin tube with frustrated diagonals as shown in Fig. 42). $\mathrm{Ba}_{3} \mathrm{Mn}_{2} \mathrm{O}_{8}$ is an example of six degenerate global minima in the single-triplet dispersion (Stone et al., 2008a). This degeneracy arises from the frustrated nature of the exchange interaction between dimers on the same and on adjacent triangular layers. The resulting dispersion is shown in Fig. 35. While single-Q ordering is observed above $H_{c 1}$ for $\mathbf{H}$ parallel to the $c$ axis (Uchida et al., 2002; Samulon et al., 2008, 2009, 2010; Stone et al., 2008a; 2009; 2010), a second phase appears right above $H_{c 1}$ for any other direction of $\mathbf{H}$. While this second phase certainly corresponds to a multi-Q structure that arises from easy-axis anisotropy in the $a-b$ plane (the condensation occurs (a)

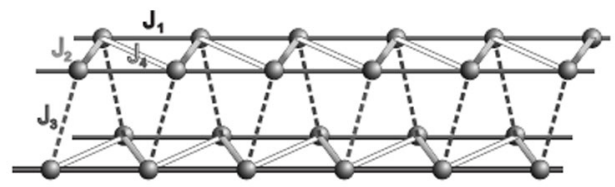

(b)

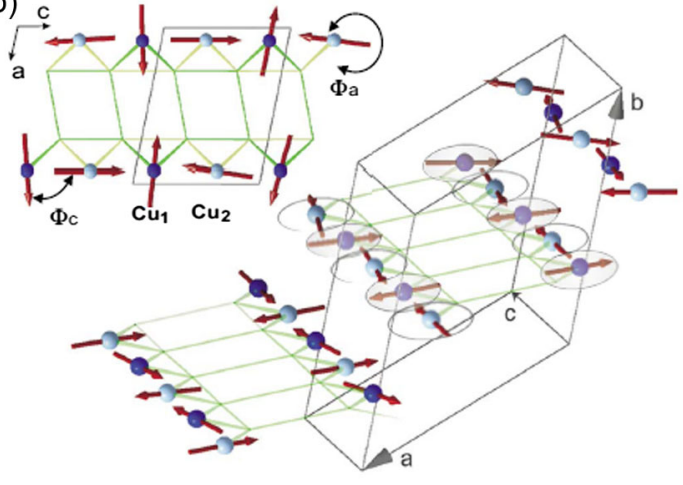

FIG. 42 (color online). (a) Schematic (not crystallographic) representation of frustration in the four-leg spin tube of Sul- $\mathrm{Cu}_{2} \mathrm{Cl}_{4}$. From Zheludev et al., 2009. (b) Incommensurate helical structure induced by magnetic fields in $\mathrm{Sul}-\mathrm{Cu}_{2} \mathrm{Cl}_{4}$ with wave vector $k=(-0.22,0,0.48)$. From Garlea et al., 2009.

simultaneously in $\mathbf{Q}$ and $-\mathbf{Q}$ so the transverse spin component can point along the easy-axis), it is still unclear if the phase is a double- $\mathbf{Q}$, four- $\mathbf{Q}$ or six- $\mathbf{Q}$ state. In particular, as it was recently demonstrated by Kamiya and Batista (2013), the six-Q structures correspond to triangular crystals of magnetic vortex with an intervortex distance that is controlled by the ratio between interlayer and intralayer exchange interactions between different spin dimers. This six- $\mathbf{Q}$ phase is favored by the symmetric exchange anisotropy terms that become relevant close enough to the critical fields because they are quadratic in the bosonic operators (the interaction terms are quartic). Magnetic vortex crystals can only arise from $n-\mathbf{Q}$ condensates with $n \geq 3$, i.e., the single-boson dispersion must have more than two minima. This observation reflects the new possibilities that are opened by magnetic realizations of bosonic gases. Atomic gases and superconducting systems normally become single $\mathbf{Q}=\mathbf{0}$ condensates because their kinetic energy terms are not frustrated (the single-boson dispersion has a single minimum). In contrast, it is rather common to find single-boson dispersions with several minima in highly frustrated magnets, which is the minimum requirement for observing exotic multi- $\mathbf{Q}$ condensates.

The combination of the two types of frustration between exchange interactions connecting the same and different pairs of dimers provides one way of stabilizing the so-called supersolid structures. The name is imported from the bosonic version of these phases in which crystal or "solid" ordering coexist with a superfluid state. The magnetic counterpart corresponds to coexistence of Ising and $X Y$ ordering. For instance, it is predicted that this phase is stabilized over a wide range of concentrations of hard-core bosons on a triangular lattice for negative hopping amplitude, $t<0$, and large enough nearest-neighbor repulsion $V \gg|t|$ (Boninsegni and Prokof'ev, 2005; Heidarian and Damle, 2005; Wessel and Troyer, 2005). These results imply that triangular layers of 
dimers, like the ones in $\mathrm{Ba}_{3} \mathrm{Mn}_{2} \mathrm{O}_{8}$, would host a spinsupersolid state if there were enough frustration between exchange interactions connecting the same pair of dimers [see Eqs. (31)]. Spin-1 Heisenberg systems with strong uniaxial and easy-axis single-ion anisotropy provide an alternative realization of supersolidity in the same effective model for hard-core bosons $(t<0$ and $V \gg|t|)$. Experimental examples of "spin supersolids" in quantum magnets have not been found yet.

\section{FUTURE DIRECTIONS AND CONCLUSIONS}

\section{A. Disorder}

Having established BEC-like quantum critical behavior in quantum magnets, we can now use these compounds to explore other frontiers in the physics of bosonic gases. This is perhaps the most important outgrowth of the study of BEC in quantum magnets-shedding light on unsolved mysteries of bosonic systems for which quantum magnets could provide an alternative route toward achieving understanding. For example, we have seen in Sec. II.C.2 that BEC QCPs do not satisfy the Harris criterion because $d \nu<2$ (Harris, 1974). This observation implies that disorder is relevant for inducing novel quantum critical behavior at the transition between the so-called Bose glass phase and the BEC. A Bose glass phase can be realized when disorder suppresses global phase coherence and the bosons are confined in localized regions of different sizes (Fisher et al., 1989). The phase transition from Bose glass to BEC (BG to BEC) can be considered analogous to Anderson localization for fermions (Giamarchi and Schulz, 1988), where conduction electron Bloch states scatter off random disorder potentials, leading to localization. Both the bosonic and the fermionic cases are of fundamental importance to understanding the effect of disorder on the physical properties of materials. Disorder causes the loss of phase coherence in bosonic systems, and a metal-to-insulator transition for fermions. Quantum magnets provide a bosonic system in which the thermodynamic properties of the BEC-BG transition can be studied in order to guide the field toward a theoretical resolution of this important problem. The BG-to-BEC phase transition has been proposed to occur in many systems including superfluids, condensates of cold atoms, and high$T_{c}$ superconductors (Kramer and MacKinnon, 1993; Emery and Kivelson, 1995; Crowell, Van Keuls, and Reppy, 1997; Azuah et al., 2003; Lye et al., 2005; Fallani, Fort, and Inguscio, 2008; Sanchez-Palencia and Lewenstein, 2010; Bouadim et al., 2011; Sacépé et al., 2011). However, quantum magnets provide a natural and convenient scenario to test the fundamental predictions for this QCP.

In quantum magnets, the most studied route to creating a Bose glass is through chemical doping. A recent review article summarizes theoretical and experimental work in this field (Zheludev and Roscilde, 2013). In the Bose glass both the amplitude and the phase of the order parameter (magnitude of ordered magnetic moment and its orientation) vary from one localized BEC cluster to the next.

Two approaches to creating a Bose glass in quantum magnets have been proposed in theory: site dilution and bond disorder. In site dilution, the magnetic ion is replaced with a nonmagnetic impurity, until a percolation threshold is reached and the transition to a Bose glass occurs (Roscilde and Haas, 2005; Roscilde, 2006, 2007; Yu, Haas, and Roscilde, 2010). Although this approach has been treated theoretically, experimental examples have not yet been found. In one experimental work, $\mathrm{V}$ was doped into the $\mathrm{Mn}$ site of $\mathrm{Ba}_{3} \mathrm{Mn}_{2} \mathrm{O}_{8}$. However, the experimental results were more consistent with a random dimer model (Samulon, Shapiro, and Fisher, 2011).

On the other hand, the bond disorder approach has had more success in experiments. Here disorder is introduced by doping on a nonmagnetic ion that is on the exchange path, resulting in randomness in the magnetic exchange between magnetic ions and/or single-ion crystal electric fields. This disorder results in different critical magnetic fields for inducing long-range order (Nohadani, Wessel, and Haas, 2005; Yu, Roscilde, and Haas, 2005; Carrasquilla, Becca, and Fabrizio, 2011; Crépin et al., 2011; Yu et al., 2012). Thus Bose glass behavior occurs in the intermediate regime of magnetic field for which the spin gaps for some magnetic ions have been suppressed to zero but not others. Bose glass behavior due to bond doping has been studied in several materials including IPA-CuCl${ }_{3-x} \mathrm{Br}_{x}$ (Saito et al., 2006; Adachi et al., 2007; Manaka, Kolomiets, and Goto, 2008; Manaka et al., 2009; Hong et al., 2010), $\mathrm{Tl}_{1-x} \mathrm{~K}_{x} \mathrm{CuCl}_{3}$ (Shindo and Tanaka, 2004; Suzuki et al., 2009, 2010; Yamada et al., 2011), and DTN (Yu et al., 2012; Wulf et al., 2013).

Measurements that suggest the presence of a Bose glass phase include: the overall phase diagram (in which Bose glass behavior appears for magnetic fields adjacent to the domeshaped region of BEC), a finite magnetic susceptibility in the absence of long-range order inside the Bose glass phase, evidence for local clusters of magnetic order in ac susceptibility, NMR measurements, ESR line shapes, and increases in the spin relaxation rates in $\mu \mathrm{SR}$ measurements. A key signature of the Bose glass phase is the critical exponent $\phi$ of the critical field versus temperature line, when the Bose glass is driven toward the BEC with magnetic fields by closing all the local gaps. This exponent $\phi$ can be determined by QMC simulations and compared to experiments. Simulations and experiments in IPA- $\mathrm{CuCl}_{3}, \mathrm{Tl}_{1-x} \mathrm{~K}_{x} \mathrm{CuCl}_{3}$, and DTN show an exponent $\phi=1$ for the critical field versus temperature line at the 3D Bose glass to BEC phase transition. This contrasts with the $\phi=3 / 2$ exponent at the quantum paramagnet to BEC in the absence of disorder (Table III). This BG-to-BEC phase transition is also marked by broadening in the phase transition in the magnetization, even though the specific heat transition remains sharp and consistent with long-range order. Analytical attempts to derive the exponent at the BG-to-BEC phase transition do not agree with the value of $\phi=1$ determined from experiments and QMC simulations. The classic prediction from analytical theory (Fisher et al., 1989 ) is that the exponent of the critical field versus temperature should be $\phi<1 / 2$. Thus, an analytical approach to the BG-to-BEC transition that agrees with experiments still needs to be developed.

One experimental challenge for studying Bose glass behavior is that chemical doping can introduce other unwanted effects like local crystalline distortions that break uniaxial 
symmetry. DTN provides an unusually clean approach to studying chemical doping because one of the $\mathrm{Cl}$ sites is oversized, and thus can accommodate a larger Br dopant without significantly changing the lattice parameters. This prevents unwanted effects like clustering of dopants or buckling of bonds that might introduce uniaxial symmetry breaking (Yu et al., 2012). The compound $\mathrm{Sul}-\mathrm{Cu}_{2} \mathrm{Cl}_{4}$ is another BEC-related material in which $\mathrm{Br}$ doping is relatively clean, producing only a $0.15 \%$ change in the $c$-axis lattice parameter with 5\% doping (Wulf et al., 2011). However, Bose glass behavior does not ensue in Sul- $\mathrm{Cu}_{2} \mathrm{Cl}_{4}$ due to frustration. In the undoped compound, the frustration along the four-leg spin tube creates an incommensurate spiral magnetic order. With $\mathrm{Br}$ doping, phase slips are introduced into the incommensurate magnetic spirals such that adjacent spin tubes are no longer in phase with each other, resulting in the intertube interactions averaging to zero. Thus three-dimensional order is suppressed with only $1 \%$ doping, which is the lowest doping level studied. We believe, however, that the resulting onedimensional behavior is interesting in and of itself. Onedimensional disordered systems could be the easiest starting point for creating contact between analytical approaches and experiment because of the powerful analytical tools that exist for treating the quasi-1D limit. Thus experimental doping studies in spin Luttinger liquid systems are important, for example, in Sul- $\mathrm{Cu}_{2} \mathrm{Cl}_{4}$ and ( $\left.\mathrm{HPIP}\right)_{2}-\mathrm{CuBr}_{4}$.

\section{B. Exotic states induced by frustration}

As discussed in previous sections, frustration can change the nature of the magnetic-field-induced QCP and the magnetic states that appear above $H_{c 1}$. The elusive spin-liquid state, in which frustration prevents the traditional symmetry breaking present in Landau phase transitions, is the paradigmatic example of an exotic state of matter. There are also rather unusual broken symmetry states that could be stabilized in frustrated quantum magnets.

Wen (2002) identified three subcategories of gapless spin liquids: those where low-level excitations have fermionic statistics like a Fermi liquid; "algebraic spin liquids," where the excitations are neither bosonic nor fermionic; and those with bosonic statistics. The last type, known as Bose metals, are predicted to have a 1D "Bose surface" analogous to Fermi surfaces, except they are not constrained by Fermi statistics.

Bose metals have been extensively studied in the context of high- $T_{c}$ superconductivity (Phillips and Dalidovich, 2003), and are proposed to explain a metallic phase that is observed between the superconducting and the insulating phases in $2 \mathrm{D}$ thin films. In quantum magnets, many theoretical microscopic models have been proposed for realizing the Bose metal, including different kinds of spin ladders (Motrunich and Fisher, 2007; Sheng et al., 2008; Sheng, Motrunich, and Fisher, 2009; Mishmash et al., 2011), 2D kagome lattices (Balents, Fisher, and Girvin, 2002; Dang, Inglis, and Melko, 2011), square lattices (Schaffer, Burkov, and Melko, 2009; Tay and Motrunich, 2011), and a 2D honeycomb lattice(Block et al., 2011; Varney et al., 2011). Here the Bose metal is of course not metallic in the sense of conducting electricity, but in the sense of conducting heat at $T=0$. Most of these Bose metal models for quantum magnets rely on ring exchange, which is a kinetic term in the Hamiltonian that favors rotating the configuration of spins around a ring, e.g., $\left|\sigma_{1}, \sigma_{2}, \sigma_{3}, \sigma_{4}\right\rangle \rightarrow\left|\sigma_{4}, \sigma_{1}, \sigma_{2}, \sigma_{3}\right\rangle$. A theory of a kagome lattice with ring exchange and up to third next-nearest-neighbor interactions (Dang, Inglis, and Melko, 2011) predicts a novel QCP at the transition between a Bose metal and long-range $X Y$ antiferromagnetic order. A Bose metal arising from a twoleg spin ladder on a triangular lattice has been proposed (among many other models) (Sheng, Motrunich, and Fisher, 2009) to explain the spin liquid phase of $\kappa-(\mathrm{Et})_{2} \mathrm{Cu}_{2}(\mathrm{CN})_{3}$ and $\mathrm{EtMe}_{3} \mathrm{Sb}\left[\mathrm{Pd}(\mathrm{dmit})_{2}\right]_{2}$. These are organic materials with tunable Mott gaps, where spin liquid behavior is observed in close proximity to a metal-to-insulator transition where the Mott gap is suppressed to zero (Kanoda and Kato, 2011).

One guiding principle for finding exotic states like spin liquids or complex magnetic orderings is to look for highly degenerate single-particle excitation minima in these quantum magnets. We have seen in the previous section that singleboson dispersions with a few global minima can lead to ordered multi- $Q$ magnetic structures right above the critical field $H_{c 1}$. On the other hand, a spin liquid could result if the single-boson dispersion has continuous manifold of global minima. However, often quantum or thermal fluctuations will remove the continuous degeneracy and select a BEC state at one or more high-symmetry wave vectors that minimize the single-boson dispersion. This selection mechanism leads to other types of exotic behavior. For instance, we have seen in Sec. II.C.2 that a line of minima at $(\pi, \pi, k)$ with $k$ taking any arbitrary value leads to dimensional reduction at a field-induced QCP, which was applied to the compound $\mathrm{BaCuSi}_{2} \mathrm{O}_{6}$ in the scenario of antiferromagnetic interlayer interactions. We have also seen that an order-from-disorder mechanism selects the BEC at $(\pi, \pi, 0)$ right above the critical field $H_{c 1}$ (Batista et al., 2007; Schmalian and Batista, 2008). Thus highly frustrated spin Hamiltonians lead to a range of exotic behavior in the quantum magnets covered in this review, and spin liquids could be realized in materials with sufficiently degenerate dispersion relations.

\section{Low dimensionality}

Besides frustration, another way to suppress long-range order in bosonic systems is to reduce the dimensionality. The Luttinger liquid is the most ubiquitous gapless phase of onedimensional systems (Sachdev, 1994; Giamarchi and Tsvelik, 1999; Ding, Yao, and Fu, 2010; Cazalilla et al., 2011; Crépin et al., 2011; Ninios et al., 2012). It can be reached in quantum magnets, for example, by closing the Haldane gap in a 1D Haldane chain with applied magnetic fields, or the spin gap in a $1 \mathrm{D}$ spin ladder. The resulting spectrum is gapless as in a $d=3 \mathrm{BEC}$, but has algebraically decaying spin correlations instead of long-range order. It also shows fractional $S=1 / 2$ spin excitations called "spinons." Two examples of spin Luttinger liquid behavior in quantum magnets are (HPIP) ${ }_{2}-\mathrm{CuBr}_{4}$ (Klanjšek et al., 2008; Rüegg et al., 2008; Thielemann, Rüegg, Kiefer et al., 2009) and $\left(\mathrm{C}_{7} \mathrm{H}_{10} \mathrm{~N}\right)_{2} \mathrm{CuBr}_{4}$ (Hong et al., 2010; Schmidiger et al., 2011, 2012; Ninios et al., 2012). These are quasi-1D spin ladder systems in which 3D ordering occurs at temperatures of 100-150 mK or below. Spin-ladder systems are a good field in which to 


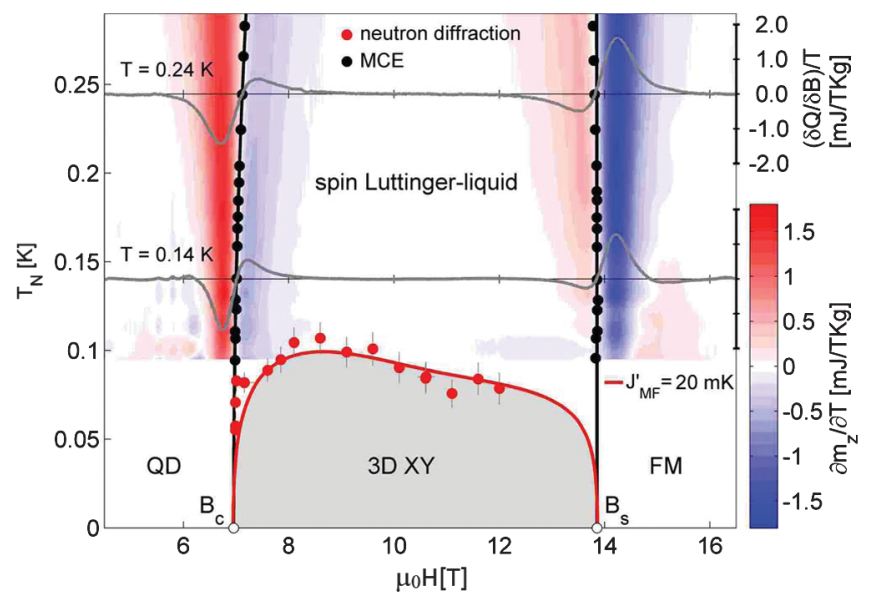

FIG. 43 (color). Phase diagram of (HPIP) $)_{2}-\mathrm{CuBr}_{4}$ showing regions of spin-Luttinger-liquid behavior and $3 \mathrm{D}$ BEC ordering at the top. The magnetocaloric effect and the magnetization derived from the magnetocaloric effect are shown in the contour plot. The line at the bottom of the plot the $T_{N}$ points is a theoretical prediction. From Thielemann, Rüegg, Kiefer et al., 2009.

search for spin Luttinger liquids because the magnetic interactions between ladders tend to be weak due to frustration. (HPIP) $)_{2}-\mathrm{CuBr}_{4}$ is in the strong-rung limit (Giamarchi and Tsvelik, 1999) with $J_{\text {leg }} / J_{\text {rung }} \approx 0.25$ whereas in $\left(\mathrm{C}_{7} \mathrm{H}_{10} \mathrm{~N}\right)_{2} \mathrm{CuBr}_{4}$ (DIMPY) the leg interactions dominate with $J_{\text {leg }} / J_{\text {rung }}=2.2$.

$(\mathrm{HPIP})_{2}-\mathrm{CuBr}_{4}$ exhibits magnetic ordering below $\sim 100 \mathrm{mK}$ in applied magnetic fields and the spinLuttinger-liquid phase can be observed between $100 \mathrm{mK}$ and $1.5 \mathrm{~K}$ (see Fig. 43). The phase diagram is in qualitative agreement with the spin-Luttinger-liquid picture, and the characteristic linear-in- $T$ specific heat is observed at low temperatures. Both the temperature and field dependence of the specific heat are in very good agreement with numerical calculations with no free parameters.

Similar results are found in DIMPY. INS results in for DIMPY are in excellent agreement with theory and no longrange order has been observed in the specific heat down to $150 \mathrm{mK}$ (Hong et al., 2010) and the magnetization down to $45 \mathrm{mK}$ (Ninios et al., 2012). We mention that IPA-CuCl 3 discussed previously is also a quasi-1D spin ladder compound with ferromagnetic rungs, but it has sufficient interladder interactions to create 3D long-range order at $10 \mathrm{~K}$, which is too high to allow for the observation of spin-Luttinger-liquid behavior for $T>T_{N}$.

\section{Other exotic states}

Finally, there are other exotic broken symmetry states that can be induced by magnetic fields such as spin supersolids (Ng and Lee, 2006; Laflorencie and Mila, 2007; Sengupta and Batista, 2007a; 2007b; Picon et al., 2008; Peters, McCulloch, and Selke, 2012), spin nematic ordering (Wierschem et al., 2012), and complex crystal or Ising-like orderings (Smith and Keszler, 1991; Kageyama, Onizuka et al., 1999; Kageyama, Yoshimura, 1999; Misguich, Jolicoeur, and Girvin, 2001;
Kodama et al., 2002; Shastry and Kumar, 2002; Miyahara and Ueda, 2003; Sebastian et al., 2008; Jaime et al., 2012).

Each of these states has its bosonic counterpart. For instance, the field-induced spin-nematic ordering corresponds to a BEC of pairs of bosons. This phase appears right below the saturation field of $S=1$ Heisenberg antiferromagnets with strong single-ion easy-axis anisotropy. Since a BEC of single bosons is more stable for lower values of the anisotropy, this kind of magnet allows for studies of the quantum phase transition between BECs of single bosons and pairs (Wierschem et al., 2012). The atomic physics counterpart of this phenomenon is the transition between BECs of atoms and diatomic molecules that may occur whenever the interatomic interaction is attractive. Moreover, the same $S=1$ model on frustrated lattices, such as triangular or face centered cubic, contains a phase in which crystal or Ising-like ordering coexists with a BEC of pairs or spin-nematic ordering for large enough anisotropy (Boninsegni and Prokof'ev, 2005; Heidarian and Damle, 2005; Wessel and Troyer, 2005; Suzuki and Kawashima, 2007) (see discussion at the end of Sec. IV). Magnets that reproduce bosonic gases with attractive interactions can also be used for observing Efimov states (Efimov, 1970; Nishida, Kato, and Batista, 2013). Spin-supersolid states are also predicted to appear for bipartite lattices of $S=1$ dimers (Sengupta and Batista, 2007a).

\section{E. Conclusions}

Quantum magnets that exhibit BEC-like behavior remain an active area of research. BEC phase transitions have now been observed in at least a dozen quantum magnets, and undoubtedly there are many more waiting to be discovered. These compounds provide realizations of gases of bosons in homogeneous (translationally invariant) systems. This is an important advantage relative to trapped atomic gases for the study of quantum phase transitions, since trapped atomic gases are subject to a trap that imposes a spatially varying potential on small spatial scales. Another advantage to quantum magnets is that they have a well-defined temperature and a macroscopic number of bosons. Thus the thermodynamic properties can be studied. A significant feature of these quantum magnets is that the particle number can be cleanly tuned with applied magnetic fields, providing a powerful system in which to study the rich variety of phases that emerge in different limits of boson densities. Most work has focused on $S=1 / 2$ dimer systems, motivated by the initial report of BEC in $\mathrm{TlCuCl}_{3}$ (Rüegg et al., 2004). However, BEC-related phenomena have also been observed in systems with higher spins and dimers of higher spins, such as DTN, $\mathrm{Ba}_{3} \mathrm{Mn}_{2} \mathrm{O}_{8}$, $\mathrm{F}_{2} \mathrm{PNNNO}$, and $\mathrm{AgVOAsO}_{4}$. Haldane-like systems, like IPA- $\mathrm{CuCl}_{3}$, have also been used to study the magneticfield-induced BEC quantum critical point. Some other interesting examples of BEC in quantum magnets, such as easyplane ferromagnets (Syromyatnikov, 2007), still remain to be explored. Finally, there are many other bosonic phases and phase transitions for which quantum magnets can give important insight. We believe that the observation of BECrelated phenomena in quantum magnets is only the beginning 
of a long journey that will reveal many interesting states and phase transitions of interacting gases of bosons.

\section{ACKNOWLEDGMENTS}

The NHMFL-PFF is funded by the U.S. National Science Foundation through Cooperative Grant No. DMR1157490, the State of Florida, and the U.S. Department of Energy. We also acknowledge support from the Laboratory-Directed Research and Development program at LANL and the U.S. Department of Energy Basic Energy Sciences "Science at 100 Tesla" project.

\section{REFERENCES}

Abrikosov, A. A., L. P. Gorkov, and I. E. Dzyaloshinski, 1975, Method of Quantum Field Theory in Statistical Physics (Dover, New York).

Aczel, A., H. Dabkowska, J. Britten, L. Harrington, and G. Luke, 2007, Acta Crystallogr. E63, I196.

Aczel, A., H. Dabkowska, P. Provencher, and G. Luke, 2008, J. Cryst. Growth 310, 870.

Aczel, A. A., Y. Kohama, M. Jaime, K. Ninios, H. B. Chan, L. Balicas, H. A. Dabkowska, and G. M. Luke, 2009, Phys. Rev. B 79, 100409.

Aczel, A. A., Y. Kohama, C. Marcenat, F. Weickert, M. Jaime, O. E. Ayala-Valenzuela, R. D. McDonald, S. D. Selesnic, H. A. Dabkowska, and G. M. Luke, 2009, Phys. Rev. Lett. 103, 207203.

Adachi, T., K. Kanada, T. Saito, A. Oosawa, and T. Goto, 2007, J. Phys. Soc. Jpn. 76, 083701

Affleck, I., 1990, Phys. Rev. B 41, 6697.

Affleck, I., 1991, Phys. Rev. B 43, 3215.

Albuquerque, A. F., N. Laflorencie, J.-D. Picon, and F. Mila, 2011, Phys. Rev. B 83, 174421.

Anderson, M. H., J. R. Ensher, M. R. Matthews, C. E. Wieman, and E. A. Cornell, 1995, Science 269, 198.

Anfuso, F., M. Garst, A. Rosch, O. Heyer, T. Lorenz, C. Rüegg, and K. Krämer, 2008, Phys. Rev. B 77, 235113.

Arlego, M., and W. Brenig, 2011, Phys. Rev. B 84, 134426.

Auerbach, A., 1998, Interacting Electrons and Quantum Magnetism (Springer, New York).

Azuah, R. T., H. R. Glyde, R. Scherm, N. Mulders, and B. Fak, 2003, J. Low Temp. Phys. 130, 557.

Balents, L., 2010, Nature (London) 464, 199.

Balents, L., M. P. A. Fisher, and S. M. Girvin, 2002, Phys. Rev. B 65, 224412.

Batista, C. D., and G. Ortiz, 2001, Phys. Rev. Lett. 86, 1082.

Batista, C. D., and G. Ortiz, 2004, Adv. Phys. 53, 1.

Batista, C. D., J. Schmalian, N. Kawashima, P. Sengupta, S. E. Sebastian, N. Harrison, M. Jaime, and I. R. Fisher, 2007, Phys. Rev. Lett. 98, 257201.

Batyev, E., and L. Braginskii, 1984, Sov. Phys. JETP 60, 781.

Beliaev, S. T., 1958, Sov. Phys. JETP 7, 299.

Blatt, J. M., K. M. Böer, and W. Brandt, 1962, Phys. Rev. 126, 1691.

Block, M. S., D. N. Sheng, O. I. Motrunich, and M. P. A. Fisher, 2011, Phys. Rev. Lett. 106, 157202.

Boninsegni, M., and N. Prokof'ev, 2005, Phys. Rev. Lett. 95, 237204.

Bose, S. N., 1924, Z. Phys. 26, 178.

Bostrem, I., S. Sinitsyn, A. Ovchinnikov, Y. Hosokoshi, and K. Inoue, 2010, J. Phys. Condens. Matter 22, 036001.
Bouadim, K., Y. L. Loh, M. Randeria, and N. Trivedi, 2011, Nat. Phys. 7, 884.

Bouillot, P., et al., 2011, Phys. Rev. B 83, 054407.

Bunkov, Y. M., E. M. Alakshin, R. R. Gazizulin, A. V. Klochkov, V. V. Kuzmin, T. R. Safin, and M. S. Tagirov, 2011, JETP Lett. 94, 68. Bunkov, Y.M., and G.E. Volovik, 2007, Phys. Rev. Lett. 98, 265302.

Bunkov, Y. M., and G. E. Volovik, 2010, J. Phys. Condens. Matter 22, 164210.

Butov, L. V., C. W. Lai, A. L. Ivanov, A. C. Gossard, and D. S. Chemla, 2002, Nature (London) 417, 47.

Butov, L. V., A. Zrenner, G. Abstreiter, G. Böhm, and G. Weimann, 1994, Phys. Rev. Lett. 73, 304.

Carrasquilla, J., F. Becca, and M. Fabrizio, 2011, Phys. Rev. B 83, 245101.

Cavadini, N., G. Heigold, W. Henggeler, A. Furrer, H.-U. Güdel, K. Krämer, and H. Mutka, 2001, Phys. Rev. B 63, 172414.

Cavadini, N., C. Rüegg, A. Furrer, H.-U. Güdel, K. Krämer, H. Mutka, and P. Vorderwisch, 2002, Phys. Rev. B 65, 132415.

Cazalilla, M. A., R. Citro, T. Giamarchi, E. Orignac, and M. Rigol, 2011, Rev. Mod. Phys. 83, 1405.

Chiatti, O., A. Sytcheva, J. Wosnitza, S. Zherlitsyn, A. A. Zvyagin, V. S. Zapf, M. Jaime, and A. Paduan-Filho, 2008, Phys. Rev. B 78, 094406.

Chiatti, O., S. Zherlitsyn, A. Sytcheva, J. Wosnitza, A. A. Zvyagin, V. S. Zapf, M. Jaime, and A. Paduan-Filho, 2009, J. Phys. Conf. Ser. 150, 042016.

Chubukov, A. V., 1989, JETP Lett. 49, 129.

Coldea, R., D. A. Tennant, K. Habicht, P. Smeibidl, C. Wolters, and Z. Tylczynski, 2002, Phys. Rev. Lett. 88, 137203.

Coldea, R., D. A. Tennant, A. M. Tsvelik, and Z. Tylczynski, 2001, Phys. Rev. Lett. 86, 1335.

Coldea, R., D. A. Tennant, and Z. Tylczynski, 2003, Phys. Rev. B 68 , 134424.

Conner, B. S., H. D. Zhou, Y. J. Jo, L. Balicas, C. R. Wiebe, J. P. Carlo, Y. J. Uemura, A. A. Aczel, T. J. Williams, and G. M. Luke, 2010, Phys. Rev. B 81, 132401.

Cox, S., R. D. McDonald, M. Armanious, P. Sengupta, and A. Paduan-Filho, 2008, Phys. Rev. Lett. 101, 087602.

Crépin, F., N. Laflorencie, G. Roux, and P. Simon, 2011, Phys. Rev. B 84, 054517.

Crowell, P. A., F. W. Van Keuls, and J. D. Reppy, 1997, Phys. Rev. B 55, 12620.

Daidouh, A., 1997, J. Solid State Chem. 130, 28.

Dalfovo, F., S. Giorgini, L. P. Pitaevskii, and S. Stringari, 1999, Rev. Mod. Phys. 71, 463.

Dang, L., S. Inglis, and R. G. Melko, 2011, Phys. Rev. B 84, 132409.

Davis, K. B., M. O. Mewes, M. R. Andrews, N. J. van Druten, D. S. Durfee, D. M. Kurn, and W. Ketterle, 1995, Phys. Rev. Lett. 75, 3969.

Demokritov, S. O., V. E. Demidov, O. Dzyapko, G. A. Melkov, A. A. Serga, B. Hillebrands, and A. N. Slavin, 2006, Nature (London) 443, 430.

Deng, H., H. Haug, and Y. Yamamoto, 2010, Rev. Mod. Phys. 82, 1489.

Ding, L.-J., K.-L. Yao, and H.-H. Fu, 2010, Chem. Phys. Chem. 11, 3291.

Efimov, V., 1970, Phys. Lett. 33B, 563.

Einstein, A., 1924, Sitz. Ber. Kgl. Preuss. Akad. Wiss. 261, 18.

Eisenstein, J. P., and A. H. MacDonald, 2004, Nature (London) 432, 691.

Emery, V. J., and S. A. Kivelson, 1995, Nature (London) 374, 434. 
Fallani, L., C. Fort, and M. Inguscio, 2008, Adv. At. Mol. Opt. Phys. 56, 119 .

Fetter, A. L., and J. D. Walecka, 2003, Quantum Theory of ManyParticle Systems (Dover, New York).

Fisher, D. S., and P. C. Hohenberg, 1988, Phys. Rev. B 37, 4936.

Fisher, M. P. A., P. B. Weichman, G. Grinstein, and D. S. Fisher, 1989, Phys. Rev. B 40, 546.

Friedberg, R., T. Lee, and H. Ren, 1993, Ann. Phys. (N.Y.) 228, 52.

Fujisawa, M., B. Kurniawan, T. Ono, and H. Tanaka, 2005, Prog. Theor. Phys. 159, 212.

Fujisawa, M., K. Shiraki, S. Okubo, H. Ohta, M. Yoshida, H. Tanaka, and T. Sakai, 2009, Phys. Rev. B 80, 012408.

Fujisawa, M., J.-I. Yamaura, H. Tanaka, H. Kageyama, Y. Narumi, and K. Kindo, 2003, J. Phys. Soc. Jpn. 72, 694.

Fukumoto, Y., 2000, J. Phys. Soc. Jpn. 69, 2755.

Furrer, A., and C. Rüegg, 2006, Physica (Amsterdam) 385B, 295.

Garlea, V. O., A. Zheludev, K. Habicht, M. Meissner, B. Grenier, L.-P. Regnault, and E. Ressouche, 2009, Phys. Rev. B 79, 060404.

Garlea, V. O., et al., 2007, Phys. Rev. Lett. 98, 167202.

Garlea, V. O., A. Zheludev, L.-P. Regnault, J.-H. Chung, Y. Qiu, M. Boehm, K. Habicht, and M. Meissner, 2008, Phys. Rev. Lett. 100, 037206.

Garst, M., and A. Rosch, 2005, Phys. Rev. B 72, 205129.

Giamarchi, T., 2004, Quantum Physics in One Dimension (Oxford University Press, Oxford, UK).

Giamarchi, T., C. Rüegg, and O. Tchernyshyov, 2008, Nat. Phys. 4, 198.

Giamarchi, T., and H. J. Schulz, 1988, Phys. Rev. B 37, 325.

Giamarchi, T., and A. M. Tsvelik, 1999, Phys. Rev. B 59, 11398.

Glazkov, V. N., A. I. Smirnov, H. Tanaka, and A. Oosawa, 2004, Phys. Rev. B 69, 184410.

Glazkov, V. N., T. S. Yankova, J. Sichelschmidt, D. Hüvonen, and A. Zheludev, 2012, Phys. Rev. B 85, 054415.

Goto, K., M. Fujisawa, T. Ono, H. Tanaka, and Y. Uwatoko, 2004, J. Phys. Soc. Jpn. 73, 3254.

Grenier, B., and T. Ziman, 2007, C.R. Phys. 8, 717.

Griffin, A., D. Snoke, and S. Stringari, 1995, Eds., Bose-Einstein Condensation (Cambridge University Press, Cambridge, England).

Haldane, F., 1983, Phys. Lett. 93A, 464.

Halperin, B., and T. Rice, 1968, Solid State Phys. 21, 115.

Hara, K., M. Inoue, S. Emori, and M. Kubo, 1971, J. Magn. Reson. 4, 337.

Harris, A. B., 1974, J. Phys. C 7, 1671.

Heidarian, D., and K. Damle, 2005, Phys. Rev. Lett. 95, 127206.

Holstein, T., and H. Primakoff, 1940, Phys. Rev. 58, 1098.

Hong, T., V. O. Garlea, A. Zheludev, J. A. Fernandez-Baca, H. Manaka, S. Chang, J. B. Leao, and S. J. Poulton, 2008, Phys. Rev. B 78, 224409.

Hong, T., C. Stock, I. Cabrera, C. Broholm, Y. Qiu, J. B. Leao, S. J. Poulton, and J.R. D. Copley, 2010, Phys. Rev. B 82, 184424.

Hosokoshi, Y., Y. Nakazawa, K. Inoue, K. Takizawa, H. Nakano, M. Takahashi, and T. Goto, 1999, Phys. Rev. B 60, 12924.

Hüvonen, D., G. Ballon, and A. Zheludev, 2013, Phys. Rev. B 88, 094402.

Inguscio, M., S. Stringari, and C. E. Wieman, 1999, in Proceedings of the International School of Physics "Enrico Fermi," Varenna, Italy (IOS Press, Amsterdam), p. 6391.

Islam, A., D. Quintero-Castro, B. Lake, K. Siemensmeyer, K. Kiefer, Y. Skourski, and T. Herrmannsdorfer, 2010, Crystal Growth and Design 10, 465.

Jackeli, G., and M.E. Zhitomirsky, 2004, Phys. Rev. Lett. 93, 017201.
Jaime, M., V. F. Correa, N. Harrison, C. D. Batista, N. Kawashima, Y. Kazuma, G. A. Jorge, R. Stern, I. Heinmaa, S. A. Zvyagin, Y. Sasago, and K. Uchinokura, 2004, Phys. Rev. Lett. 93, 087203.

Jaime, M., R. Daou, S. A. Crooker, F. Weickert, A. Uchida, A. E. Feiguin, C. D. Batista, H. A. Dabkowska, and B. D. Gaulin, 2012, Proc. Natl. Acad. Sci. U.S.A. 109, 12404.

Jaime, M., R. Movshovich, G. R. Stewart, W. P. Beyermann, M. G. Berisso, M. F. Hundley, P. C. Canfield, and J. L. Sarrao, 2000, Nature (London) 405, 160.

Johannsen, N., A. Vasiliev, A. Oosawa, H. Tanaka, and T. Lorenz, 2005, Phys. Rev. Lett. 95, 017205.

Jordan, P., and E. Wigner, 1928, Z. Phys. A 47, 631.

Kageyama, H., T. Kitano et al., 2005, J. Phys. Soc. Jpn. 74, 1702.

Kageyama, H., M. Nishi, N. Aso, K. Onizuka, T. Yosihama, K. Nukui, K. Kodama, K. Kakurai, and Y. Ueda, 2000, Phys. Rev. Lett. 84, 5876.

Kageyama, H., K. Onizuka, T. Yamauchi, Y. Ueda, S. Hane, H. Mitamura, T. Goto, K. Yoshimura, and K. Kosuge, 1999, J. Phys. Soc. Jpn. 68, 1821.

Kageyama, H., J. Yasuda et al., 2005, J. Phys. Soc. Jpn. 74, 3155 .

Kageyama, H., K. Yoshimura, R. Stern, N. V. Mushnikov, K. Onizuka, M. Kato, K. Kosuge, C. P. Slichter, T. Goto, and Y. Ueda, 1999, Phys. Rev. Lett. 82, 3168.

Kamiya, Y., and C. D. Batista, 2014, Phys. Rev. X 4, 011023.

Kanoda, K., and R. Kato, 2011, Annu. Rev. Condens. Matter Phys. 2, 167.

Kato, T., K. Takatsu, H. Tanaka, W. Shiramura, M. Mori, K. Nakajima, and K. Kakurai, 1998, J. Phys. Soc. Jpn. 67, 752.

Kawamata, T., N. Sugawara, M. Uesaka, N. Kaneko, T. Kajiwara, H. Yamane, K. Koyama, K. Kudo, N. Kobayashi, and Y. Koike, 2009, J. Phys. Conf. Ser. 150, 042087.

Kawashima, N., 2005, J. Phys. Soc. Jpn. 74S, 145.

Kawashima, N., and K. Harada, 2004, J. Phys. Soc. Jpn. 73, 1379.

Ke, W. P., X. M. Wang, C. Fan, Z. Y. Zhao, X. G. Liu, L. M. Chen, Q. J. Li, X. Zhao, and X. F. Sun, 2011, Phys. Rev. B 84, 094440.

Keldysh, L. V., and Y. V. Kopaev, 1964, Fiz. Tverd. Tela (Leningrad) 6, 2791.

Kitada, A., Z. Hiroi, Y. Tsujimoto, T. Kitano, H. Kageyama, Y. Ajiro, and K. Yoshimura, 2007, J. Phys. Soc. Jpn. 76, 093706.

Klanjšek, M., et al., 2008, Phys. Rev. Lett. 101, 137207.

Knetter, C., A. Bühler, E. Müller-Hartmann, and G. S. Uhrig, 2000, Phys. Rev. Lett. 85, 3958.

Kodama, K., M. Takigawa, M. Horvati, C. Berthier, H. Kageyama, Y. Ueda, S. Miyahara, F. Becca, and F. Mila, 2002, Science 298, 395.

Kodama, M., M. Yoshida, S. Okubo, H. Ohta, T. Waki, Y. Morimoto, C. Michioka, M. Kato, and K. Yoshimura, 2005, Prog. Theor. Phys. Suppl. 159, 114.

Kofu, M., J.-H. Kim, S. Ji, S.-H. Lee, H. Ueda, Y. Qiu, H.-J. Kang, M. A. Green, and Y. Ueda, 2009, Phys. Rev. Lett. 102, 037206.

Kofu, M., H. Ueda et al., 2009, Phys. Rev. Lett. 102, 177204.

Kohama, Y., C. Marcenat, T. Klein, and M. Jaime, 2010, Rev. Sci. Instrum. 81, 104902.

Kohama, Y., et al., 2011, Phys. Rev. Lett. 106, 037203.

Kolezhuk, A. K., V. N. Glazkov, H. Tanaka, and A. Oosawa, 2004, Phys. Rev. B 70, 020403.

Kramer, B., and A. MacKinnon, 1993, Rep. Prog. Phys. 56, 1469.

Krämer, S., N. Laflorencie, R. Stern, M. Horvatić, C. Berthier, H. Nakamura, T. Kimura, and F. Mila, 2013, Phys. Rev. B 87, 180405

Krämer, S., R. Stern, M. Horvatić, C. Berthier, T. Kimura, and I. R. Fisher, 2007, Phys. Rev. B 76, 100406. 
Kudo, K., M. Yamazaki, T. Kawamata, T. Noji, Y. Koike, T. Nishizaki, B. Kobayashi, and T. H., 2004, J. Phys. Soc. Jpn. 73, 2358.

Kühner, T. D., and S. R. White, 1999, Phys. Rev. B 60, 335.

Laflorencie, N., and F. Mila, 2007, Phys. Rev. Lett. 99, 027202.

Laflorencie, N., and F. Mila, 2009, Phys. Rev. Lett. 102, 060602.

Leggett, A. J., 2001, Rev. Mod. Phys. 73, 307.

London, F., 1938, Phys. Rev. 54, 947.

Lorenz, T., O. Heyer, M. Garst, F. Anfuso, A. Rosch, C. Rüegg, and K. Krämer, 2008, Phys. Rev. Lett. 100, 067208.

Lye, J. E., L. Fallani, M. Modugno, D. S. Wiersma, C. Fort, and M. Inguscio, 2005, Phys. Rev. Lett. 95, 070401.

Maltseva, M., and P. Coleman, 2005, Phys. Rev. B 72, 174415.

Manaka, H., H. A. Katori, O. V. Kolomiets, and T. Goto, 2009, Phys. Rev. B 79, 092401.

Manaka, H., A. V. Kolomiets, and T. Goto, 2008, Phys. Rev. Lett. 101, 077204.

Manaka, H., I. Yamada, M. Hagiwara, and M. Tokunaga, 2001, Phys. Rev. B 63, 144428.

Manaka, H., I. Yamada, and W. Higemoto, 2007, J. Phys. Soc. Jpn. 76, 014704.

Manaka, H., I. Yamada, Z. Honda, K. H. Aruga, and K. Katsumata, 1998, J. Phys. Soc. Jpn. 67, 3913.

Manaka, H., I. Yamada, N. V. Mushnikov, and T. Goto, 2000, J. Phys. Soc. Jpn. 69, 675.

Mani, R. G., J. H. Smet, K. von Klitzing, V. Narayanamurti, W. B. Johnson, and V. Umanskyk, 2002, Nature (London) 420, 646.

Manmana, S. R., J.-D. Picon, K. P. Schmidt, and F. Mila, 2011, Europhys. Lett. 94, 67004.

Masuda, T., A. Zheludev, H. Manaka, L.-P. Regnault, J.-H. Chung, and Y. Qiu, 2006, Phys. Rev. Lett. 96, 047210.

Matsubara, T., and H. Matsuda, 1956, Prog. Theor. Phys. 16, 569.

Matsumoto, M., B. Normand, T. M. Rice, and M. Sigrist, 2002, Phys. Rev. Lett. 89, 077203.

Matsumoto, M., B. Normand, T. M. Rice, and M. Sigrist, 2004, Phys. Rev. B 69, 054423.

Matsumoto, M., and M. Sigrist, 2005, J. Phys. Soc. Jpn. 74, 2310.

Mazurenko, V. V., M. V. Valentyuk, R. Stern, and A. A. Tsirlin, 2014, Phys. Rev. Lett., 112, 107202.

Misguich, G., T. Jolicoeur, and S. M. Girvin, 2001, Phys. Rev. Lett. 87, 097203

Misguich, G., and M. Oshikawa, 2004, J. Phys. Soc. Jpn. 73, 3429.

Mishmash, R. V., M. S. Block, R. K. Kaul, D. N. Sheng, O. I. Motrunich, and M.P.A. Fisher, 2011, Phys. Rev. B 84, 245127.

Miyahara, S., J.-B. Fouet, S. R. Manmana, R. M. Noack, H. Mayaffre, I. Sheikin, C. Berthier, and F. Mila, 2007, Phys. Rev. B 75, 184402.

Miyahara, S., and K. Ueda, 2003, J. Phys. Condens. Matter 15, R327.

Miyashita, S., 1997, J. Phys. Soc. Jpn. 66, 3411.

Moriya, T., 1960, Phys. Rev. 120, 91.

Motrunich, O.I., and M.P. A. Fisher, 2007, Phys. Rev. B 75, 235116.

Mott, N. F., 1961, Philos. Mag. 6, 287.

Moukouri, S., 2008, Phys. Rev. B 78, 132405.

Mukhopadhyay, S., et al., 2012, Phys. Rev. Lett. 109, 177206.

Muniz, R. A., Y. Kato, and C. D. Batista, 2013, arXiv:1307.7731.

Nakajima, T., H. Mitamura, and Y. Ueda, 2006, J. Phys. Soc. Jpn. 75, 054706.

Nandi, D., A. D. K. Finck, J. P. Eisenstein, L. N. Pfeiffer, and K. W. West, 2012, Nature (London) 488, 481.
Nawa, K., C. Michioka, K. Yoshimura, A. Matsuo, and K. Kindo, 2011, J. Phys. Soc. Jpn. 80, 034710.

Ng, K.-K., and T. K. Lee, 2006, Phys. Rev. Lett. 97, 127204.

Nikuni, T., M. Oshikawa, A. Oosawa, and H. Tanaka, 2000, Phys. Rev. Lett. 84, 5868.

Nikuni, T., and H. Shiba, 1995, J. Phys. Soc. Jpn. 64, 3471.

Ninios, K., T. Hong, T. Manabe, C. Hotta, S. N. Herringer, M. M. Turnbull, C. P. Landee, Y. Takano, and H. B. Chan, 2012, Phys. Rev. Lett. 108, 097201.

Nishida, Y., Y. Kato, and C. D. Batista, 2013, Nat. Phys. 9, 93.

Nohadani, O., S. Wessel, and S. Haas, 2005, Phys. Rev. B 72, 024440 .

Nohadani, O., S. Wessel, B. Normand, and S. Haas, 2004, Phys. Rev. B 69, 220402.

Nozieres, P., and D. Pines, 1990, The Theory of Quantum Liquids (Addison-Wesley, Reading, MA), Vol. II.

Oosawa, A., M. Fujisawa, T. Osakabe, K. Kakurai, and H. Tanaka, 2003, J. Phys. Soc. Jpn. 72, 1026.

Oosawa, A., M. Ishii, and H. Tanaka, 1999, J. Phys. Condens. Matter 11, 265

Oosawa, A., K. Kakurai, T. Osakabe, M. Nakamura, M. Takeda, and H. Tanaka, 2004, J. Phys. Soc. Jpn. 73, 1446.

Oosawa, A., T. Kato, H. Tanaka, K. Kakurai, M. Müller, and H.-J. Mikeska, 2002, Phys. Rev. B 65, 094426.

Oosawa, A., H. A. Katori, and H. Tanaka, 2001, Phys. Rev. B 63, 134416.

Orignac, E., and R. Citro, 2005, Phys. Rev. B 71, 214419.

Orignac, E., R. Citro, and T. Giamarchi, 2007, Phys. Rev. B 75, 140403

Oshikawa, M., and I. Affleck, 1997, Phys. Rev. Lett. 79, 2883.

Paduan-Filho, A., 2012, Braz. J. Phys. 42, 292.

Paduan-Filho, A., K. Al-Hassanieh, P. Segupta, V. Zapf, M. Jaime, A. Lacerda, and M. Kenzelmann, 2009, J. Appl. Phys. 105, 07D501.

Paduan-Filho, A., R. Chirico, K. Joung, and R. Carlin, 1981, J. Chem. Phys. 74, 4103.

Paduan-Filho, A., X. Gratens, and N. F. Oliveira, Jr., 2004a, J. Appl. Phys. 95, 7537.

Paduan-Filho, A., X. Gratens, and N. F. Oliveira, Jr., 2004b, Phys. Rev. B 69, 020405.

Patyal, B. R., B. L. Scott, and R. D. Willett, 1990, Phys. Rev. B 41, 1657. Peters, D., I. P. McCulloch, and W. Selke, 2012, Phys. Rev. B 85, 054423.

Pethick, C. J., and H. Smith, 2002, Bose-Einstein Condensation in Dilute Gases (Cambridge University Press, New York).

Phillips, P., and D. Dalidovich, 2003, Science 302, 243.

Picon, J.-D., A. Albuquerque, K. Schmidt, N. Laflorencie, M. Troyer, and F. Mila, 2008, Phys. Rev. B 78, 184418.

Pitaevskii, L. P., and S. Stringari, 2003, Bose-Einstein Condensation (Oxford University Press, New York).

Pleimling, M., B. Neubert, and R. Siems, 1998, J. Phys. A 31, 4871.

Popov, V. N., 1987, Functional Integrals and Collective Excitations (Cambridge University Press, Cambridge).

Povarov, K. Y., A. I. Smirnov, O. A. Starykh, S. V. Petrov, and A. Y. Shapiro, 2011, Phys. Rev. Lett. 107, 037204.

Prokofev, N., B. Svistunov, and I. Tupitsyn, 1998, J. Exp. Theor. Phys. 87, 310.

Psaroudaki, C., S. A. Zvyagin, J. Krzystek, A. Paduan-Filho, X. Zotos, and N. Papanicolaou, 2012, Phys. Rev. B 85, 014412.

Quintero-Castro, D. L., B. Lake, E. M. Wheeler, A. T. M. N. Islam, T. Guidi, K. C. Rule, Z. Izaola, M. Russina, K. Kiefer, and Y. Skourski, 2010, Phys. Rev. B 81, 014415. 
Radu, T., H. Wilhelm, V. Yushankhai, D. Kovrizhin, R. Coldea, Z. Tylczynski, T. Lühmann, and F. Steglich, 2005, Phys. Rev. Lett. 95, 127202.

Radu, T., H. Wilhelm, V. Yushankhai, D. Kovrizhin, R. Coldea, Z. Tylczynski, T. Lühmann, and F. Steglich, 2006, Phys. Rev. Lett. 96, 189704.

Reyes, D., A. Paduan-Filho, and M. A. Continentino, 2008, Phys. Rev. B 77, 052405.

Rice, T. M., 2002, Science 298, 760.

Rösch, O., and M. Vojta, 2007, Phys. Rev. B 76, 180401.

Roscilde, T., 2006, Phys. Rev. B 74, 144418.

Roscilde, T., and S. Haas, 2005, Phys. Rev. Lett. 95, 207206.

Roscilde, T., and S. Haas, 2007, Phys. Rev. Lett. 99, 047205.

Rüegg, C., N. Cavadini, A. Furrer, H.-U. Güdel, K. Krämer, H. Mutka, A. Wildes, K. Habicht, and P. Vorderwisch, 2003, Nature (London) 423, 62.

Rüegg, C., A. Furrer, D. Sheptyakov, T. Strässle, K. W. Krämer, H.-U. Güdel, and L. Mélési, 2004, Phys. Rev. Lett. 93, 257201.

Rüegg, C., D. F. McMorrow, B. Normand, H. M. Rønnow, S. E. Sebastian, I. R. Fisher, C. D. Batista, S. N. Gvasaliya, C. Niedermayer, and J. Stahn, 2007, Phys. Rev. Lett. 98, 017202.

Rüegg, C., B. Normand, M. Matsumoto, A. Furrer, D. F. McMorrow, K. W. Krämer, H. U. Güdel, S. N. Gvasaliya, H. Mutka, and M. Boehm, 2008, Phys. Rev. Lett. 100, 205701.

Sacépé, B., T. Dubouchet, C. Chapelier, M. Sanquer, M. Ovadia, D. Shahar, M. Feigelman, and L. Ioffe, 2011, Nat. Phys. 7, 239.

Sachdev, S., 1994, Z. Phys. B 94, 469.

Sachdev, S., 1999, Quantum Phase Transitions (Cambridge University Press, Cambridge, England).

Sachdev, S., and R. N. Bhatt, 1990, Phys. Rev. B 41, 9323.

Saito, T., A. Oosawa, T. Goto, T. Suzuki, and I. Watanabe, 2006, Phys. Rev. B 74, 134423.

Samulon, E. C., K. A. Al-Hassanieh, Y.-J. Jo, M. C. Shapiro, L. Balicas, C. D. Batista, and I. R. Fisher, 2010, Phys. Rev. B 81, 104421.

Samulon, E. C., Z. Islam, S.E. Sebastian, P. B. Brooks, M. K. McCourt, J. Ilavsky, and I. R. Fisher, 2006, Phys. Rev. B 73, 100407.

Samulon, E. C., Y.-J. Jo, P. Sengupta, C. D. Batista, M. Jaime, L. Balicas, and I. R. Fisher, 2008, Phys. Rev. B 77, 214441.

Samulon, E. C., Y. Kohama, R. D. McDonald, M. C. Shapiro, K. A. Al-Hassanieh, C. D. Batista, M. Jaime, and I. R. Fisher, 2009, Phys. Rev. Lett. 103, 047202.

Samulon, E. C., M. C. Shapiro, and I. R. Fisher, 2011, Phys. Rev. B 84, 054417.

Sanchez-Palencia, L., and M. Lewenstein, 2010, Nat. Phys. 6, 87.

Sandvik, A. W., 1998, Phys. Rev. B 57, 10287.

Sandvik, A. W., 1999, Phys. Rev. B 59, R14157.

Sasago, Y., K. Uchinokura, A. Zheludev, and G. Shirane, 1997, Phys. Rev. B 55, 8357.

Sato, M., T. Kawamata, N. Sugawara, N. Kaneko, M. Uesaka, K. Kudo, N. Kobayashi, and Y. Koike, 2010, J. Phys. Conf. Ser. 200, 022054

Schaffer, R., A. A. Burkov, and R. G. Melko, 2009, Phys. Rev. B 80, 014503.

Schmalian, J., and C. D. Batista, 2008, Phys. Rev. B 77, 094406.

Schmidiger, D., P. Bouillot, S. Mühlbauer, S. Gvasaliya, C. Kollath, T. Giamarchi, and A. Zheludev, 2012, Phys. Rev. Lett. 108, 167201.

Schmidiger, D., S. Mühlbauer, S. N. Gvasaliya, T. Yankova, and A. Zheludev, 2011, Phys. Rev. B 84, 144421.

Schmidt, K. P., J. Dorier, A. M. Läuchli, and F. Mila, 2008, Phys. Rev. Lett. 100, 090401.
Schrama, J., A. Ardavan, A. Semeno, P. Gee, E. Rzepniewski, J. Suto, R. Coldea, J. Singleton, and P. Goy, 1998, Physica (Amsterdam) 256-258B, 637.

Sebastian, S., N. Harrison, C. Batista, L. Balicas, M. Jaime, P. Sharma, N. Kawashima, and I. Fisher, 2006, Nature (London) 441, 617.

Sebastian, S. E., N. Harrison, P. Sengupta, C. D. Batista, S. Francoual, E. Palm, T. Murphy, N. Marcano, H. A. Dabkowska, and B. D. Gaulin, 2008, Proc. Natl. Acad. Sci. U.S.A. 105, 20157.

Sebastian, S. E., P. A. Sharma, M. Jaime, N. Harrison, V. Correa, L. Balicas, N. Kawashima, C. D. Batista, and I. R. Fisher, 2005, Phys. Rev. B 72, 100404.

Sebastian, S. E., P. Tanedo et al., 2006, Phys. Rev. B 74, 180401. Sengupta, P., and C. Batista, 2007a, Phys. Rev. Lett. 99, 217205.

Sengupta, P., and C. D. Batista, 2007b, Phys. Rev. Lett. 98, 227201.

Seradjeh, B., J. E. Moore, and M. Franz, 2009, Phys. Rev. Lett. 103, 066402.

Shastry, B. S., and B. Kumar, 2002, Prog. Theor. Phys. Suppl. 145, 1. Shastry, B. S., and B. Sutherland, 1981, Physica (Amsterdam) 108B $+\mathrm{C}, 1069$.

Sheng, D. N., O. I. Motrunich, and M. P. A. Fisher, 2009, Phys. Rev. B 79, 205112.

Sheng, D. N., O. I. Motrunich, S. Trebst, E. Gull, and M. P. A. Fisher, 2008, Phys. Rev. B 78, 054520.

Shindo, Y., and H. Tanaka, 2004, J. Phys. Soc. Jpn. 73, 2642.

Shiramura, W., K. Takatsu, H. Tanaka, K. Kamishima, M. Takahashi, H. Mitamura, and T. Goto, 1997, J. Phys. Soc. Jpn. 66, 1900.

Siegert, M., and H. U. Everts, 1989, J. Phys. A 22, L783.

Silhanek, A. V., et al., 2006, Phys. Rev. Lett. 96, 136403.

Singh, Y., and D. C. Johnston, 2007, Phys. Rev. B 76, 012407.

Sirker, J., A. Weiße, and O. P. Sushkov, 2004, Europhys. Lett. 68, 275.

Smith, R. W., and D. A. Keszler, 1991, J. Solid State Chem. 93, 430. Snoke, D., 2002, Science 298, 1368.

Starykh, O. A., H. Katsura, and L. Balents, 2010, Phys. Rev. B 82, 014421.

Stone, M. B., C. Broholm, D. H. Reich, P. Schiffer, O. Tchernyshyov, P. Vorderwisch, and N. Harrison, 2007, New J. Phys. 9, 31.

Stone, M. B., M. D. Lumsden, S. Chang, E. C. Samulon, C. D. Batista, and I. R. Fisher, 2008a, Phys. Rev. Lett. 100, 237201.

Stone, M. B., M. D. Lumsden, S. Chang, E. C. Samulon, C. D. Batista, and I. R. Fisher, 2008b, Phys. Rev. Lett. 100, 237201.

Stone, M. B., M. D. Lumsden, Y. Qiu, E. C. Samulon, C. D. Batista, and I. R. Fisher, 2008c, Phys. Rev. B 77, 134406.

Stone, M. B., I. A. Zaliznyak, T. Hong, C. L. Broholm, and D. H. Reich, 2006, Nature (London) 440, 187.

Suh, S., K. A. Al-Hassanieh, E. C. Samulon, I. R. Fisher, S.E. Brown, and C. D. Batista, 2011, Phys. Rev. B 84, 054413.

Sun, X. F., W. Tao, X. M. Wang, and C. Fan, 2009, Phys. Rev. Lett. 102, 167202.

Suzuki, T., and N. Kawashima, 2007, Phys. Rev. B 75, 180502.

Suzuki, T., F. Yamada, T. Kawamata, I. Watanabe, T. Goto, and H. Tanaka, 2009, Phys. Rev. B 79, 104409.

Suzuki, T., F. Yamada, M. Yamada, T. Kawamata, Y. Ishii, I. Watanabe, T. Goto, and H. Tanaka, 2010, Phys. Rev. B 82, 094447.

Svensson, E. C., and V. F. Sears, 1987, Progress in Low Temperature Physics (North Holland, Amsterdam), Vol. XI.

Syljuåsen, O. F., and A. W. Sandvik, 2002, Phys. Rev. E 66, 046701. Syromyatnikov, A. V., 2007, Phys. Rev. B 75, 134421.

Takatsu, K., W. Shiramura, H. Tanaka, T. Kambe, H. Nojiri, and M. Motokawa, 1998, J. Magn. Magn. Mater. 177, 697. 
Tanaka, H., A. Oosawa, T. Kato, H. Uekusa, Y. Ohashi, K. Kakurai, and A. Hoser, 2001, J. Phys. Soc. Jpn. 70, 939.

Tanaka, H., K. Takatsu, W. Shiramura, T. Ono, T. Kambe, K. Kamishima, H. Mitamura, and T. Goto, 1997, Physica (Amsterdam) 237-238B, 120.

Tanaka, H., T. Takatsu, W. Shiramura, T. Kambe, H. Nojiri, T. Yamada, S. Okubo, H. Ohta, and M. Motokawa, 1998, Physica (Amsterdam) 246B, 545.

Tanaka, H., F. Yamada, T. Ono, T. Sakakibara, Y. Uwatoko, A. Oosawa, K. Kakurai, and K. Goto, 2007, J. Magn. Magn. Mater. 310, 1343.

Tay, T., and O. I. Motrunich, 2011, Phys. Rev. B 83, 205107.

Thielemann, B., C. Rüegg, K. Kiefer et al., 2009, Phys. Rev. B 79, 020408.

Thielemann, B., C. Rüegg, H. M. Rønnow et al., 2009, Phys. Rev. Lett. 102, 107204.

Tokiwa, Y., T. Radu, R. Coldea, H. Wilhelm, Z. Tylczynski, and F. Steglich, 2006, Phys. Rev. B 73, 134414.

Totsuka, K., S. Miyahara, and K. Ueda, 2001, Phys. Rev. Lett. 86, 520.

Tsirlin, A., R. Nath, F. Weickert, Y. Skourski, C. Geibel, and H. Rosner, 2009, J. Phys. Conf. Ser. 145, 012067.

Tsirlin, A. A., R. Nath, C. Geibel, and H. Rosner, 2008, Phys. Rev. B 77, 104436

Tsirlin, A. A., R. Nath, J. Sichelschmidt, Y. Skourski, C. Geibel, and H. Rosner, 2011, Phys. Rev. B 83, 144412.

Tsirlin, A. A., and H. Rosner, 2010, Phys. Rev. B 82, 060409.

Tsujii, H., B. Andraka, Y. Hosokoshi, K. Inoue, and Y. Takano, 2007, J. Magn. Magn. Mater. 310, e415.

Tsyrulin, N., et al., 2013, J. Phys. Condens. Matter 25, 216008.

Uchida, M., H. Tanaka, H. Mitamura, F. Ishikawa, and T. Goto, 2002, Phys. Rev. B 66, 054429.

Ueda, H. T., and K. Totsuka, 2009, Phys. Rev. B 80, 014417.

Vachon, M.-A., W. Kundhikanjana, A. Straub, V. Mitrovic, A. Reyes, P. Kuhns, R. Coldea, and Z. Tylczynski, 2006, New J. Phys. 8, 222.

Varney, C. N., K. Sun, V. Galitski, and M. Rigol, 2011, Phys. Rev. Lett. 107, 077201.

Villain, J., R. Bidaux, J. P. Carton, and R. Conte, 1980, J. Phys. (Paris) 41, 1263.

Vyaselev, O., M. Takigawa, A. Vasiliev, A. Oosawa, and H. Tanaka, 2004, Phys. Rev. Lett. 92, 207202.

Wachter, P., B. Bucher, and J. Malar, 2004, Phys. Rev. B 69, 094502. Waki, T., M. Kato, Y. Itoh, C. Michioka, K. Yoshimura, and T. Goto, 2005, J. Phys. Chem. Solids 66, 1432.

Waki, T., C. Michioka, Y. Itoh, and K. Yoshimura, 2007, J. Magn. Magn. Mater. 310, 1349.

Waki, T., Y. Morimoto, M. Kato, K. Yoshimura, H. Mitamura, and T. Goto, 2005, Physica (Amsterdam) 359-361B, 1372.

Waki, T., et al., 2004, J. Phys. Soc. Jpn. 73, 3435.

Waki, T., N. Tsujii, Y. Itoh, C. Michioka, K. Yoshimura, O. Suzuki, H. Kitazawa, and G. Kido, 2007, Physica (Amsterdam) 398B, 148. Weickert, F., et al., 2012, Phys. Rev. B 85, 184408.

Weickert, F., R. Küchler, V. Zapf, M. Jaime, and A. Paduan-Filho, 2011, Phys. Rev. B 83, 099901.

Wen, X.-G., 2002, Phys. Rev. B 65, 165113.
Wessel, S., and M. Troyer, 2005, Phys. Rev. Lett. 95, 127205.

White, J. L., et al., 2010, Phys. Rev. B 81, 052407.

White, S. R., 1992, Phys. Rev. Lett. 69, 2863.

Wierschem, K., Y. Kato, Y. Nishida, C.D. B. Batista, and P. Sengupta, 2012, Phys. Rev. B 86, 201108.

Wolf, B., et al., 2011, Natl. Acad. Sci. lett. 108, 6862.

Wulf, E., D. Huevonen, J.-W. Kim, A. Paduan-Filho, E. Ressouche, S. Gvasaliya, V. Zapf, and A. Zheludev, 2013, Phys. Rev. B 88, 174418.

Wulf, E., S. Mühlbauer, T. Yankova, and A. Zheludev, 2011, Phys. Rev. B 84, 174414.

Yamada, F., T. Ono, M. Fujisawa, H. Tanaka, and T. Sakakibara, 2007, J. Magn. Magn. Mater. 310, 1352.

Yamada, F., T. Ono, H. Tanaka, G. Misguich, M. Oshikawa, and T. Sakakibara, 2008, J. Phys. Soc. Jpn. 77, 013701.

Yamada, F., H. Tanaka, T. Ono, and H. Nojiri, 2011, Phys. Rev. B 83, 020409

Yin, L., J. S. Xia, V. S. Zapf, N. S. Sullivan, and A. Paduan-Filho, 2008, Phys. Rev. Lett. 101, 187205.

Yoshida, M., N. Ogota, M. Takigawa, J. Yamaura, M. Ichihara, T. Kitano, H. Kageyama, Y. Ajiro, and K. Yoshimura, 2007, J. Phys. Soc. Jpn. 76, 104703.

Yu, R., S. Haas, and T. Roscilde, 2010, Europhys. Lett. 89, 10009. Yu, R., T. Roscilde, and S. Haas, 2005, Phys. Rev. Lett. 94, 197204. Yu, R., et al., 2012, Nature (London) 489, 379.

Zapf, V. S., et al., 2008, Phys. Rev. B 77, 020404.

Zapf, V. S., P. Sengupta, C. D. Batista, F. Nasreen, F. Wolff-Fabris, and A. Paduan-Filho, 2011, Phys. Rev. B 83, 140405.

Zapf, V. S., D. Zocco, B. R. Hansen, M. Jaime, N. Harrison, C. D. Batista, M. Kenzelmann, C. Niedermayer, A. Lacerda, and A. Paduan-Filho, 2006, Phys. Rev. Lett. 96, 077204.

Zenmoto, T., Y. Oshima, H. Nojiri, S. Lee, and M. Kofu, 2010, J. Low Temp. Phys. 159, 118.

Zhang, Z., K. Wierschem, I. Yap, Y. Kato, C. D. Batista, and P. Sengupta, 2013, Phys. Rev. B 87, 174405.

Zheludev, A., et al., 2007, Phys. Rev. B 76, 054450.

Zheludev, A., V. O. Garlea, L.-P. Regnault, H. Manaka, A. Tsvelik, and J.-H. Chung, 2008, Phys. Rev. Lett. 100, 157204.

Zheludev, A., V. O. Garlea, A. Tsvelik, L.-P. Regnault, K. Habicht, K. Kiefer, and B. Roessli, 2009, Phys. Rev. B 80, 214413.

Zheludev, A., and T. Roscilde, 2013, C.R. Phys. 14, 740.

Zherlitsyn, S., O. Chiatti, A. Sytcheva, J. Wosnitza, A. A. Zvyagin, V. S. Zapf, M. Jaime, and A. Paduan-Filho, 2009, J. Phys. Conf. Ser. 145, 012069.

Zhitomirsky, M. E., and A. L. Chernyshev, 2013, Rev. Mod. Phys. 85, 219.

Zvyagin, S. A., J. Wosnitza, C. D. Batista, M. Tsukamoto, N. Kawashima, J. Krzystek, V. S. Zapf, M. Jaime, N. F. Oliveira, and A. Paduan-Filho, 2007, Phys. Rev. Lett. 98, 047205.

Zvyagin, S. A., J. Wosnitza, A. K. Kolezhuk, V. S. Zapf, M. Jaime, A. Paduan-Filho, V. N. Glazkov, S. S. Sosin, and A. I. Smirnov, 2008, Phys. Rev. B 77, 092413.

Zvyagin, S. A., J. Wosnitza, J. Krzystek, R. Stern, M. Jaime, Y. Sasago, and K. Uchinokura, 2006, Phys. Rev. B 73, 094446. 